\title{
AdvanCEd Gasification By-Product Utilization
}

FINAL TECHNICAL REPORT

for period

1 September 2003 through 31 August 2006

prepared by

Dr. Rodney Andrews, Ms. Aurora Rubel, Dr. Jack Groppo, Mr. Brock Marrs,

Dr. Ari Geertsema

University of Kentucky Center for Applied Energy Research

Dr. Frank Huggins

University of Kentucky Center for Fossil Fuel Science

Dr. M. Mercedes Maroto-Valer, Ms. Brandie M. Markley, Ms. Zhe Lu, Dr. Harold Schobert

Pennsylvania State University Energy Institute

issued

November 2006

revised

February 2007

for contract

DE-FG26-03NT41795

submitted by

University of Kentucky Research Foundation

Lexington, $\mathrm{KY}$ 


\section{Disclaimer}

This report was prepared as an account of work sponsored by an agency of the United States Government. Neither the United States Government nor any agency thereof, nor any of their employees, makes any warranty, express or implied, or assumes any legal liability or responsibility for the accuracy, completeness, or usefulness of any information, apparatus, product, or process disclosed, or represents that its use would not infringe privately owned rights. Reference herein to any specific commercial product, process, or service by trade name, trademark, manufacturer, or otherwise does not necessarily constitute or imply its endorsement, recommendation, or favoring by the United States Government or any agency thereof. The views and opinions of authors expressed herein do not necessarily state or reflect those of the United States Government or any agency thereof. 


\section{EXECUTIVE SUMMARY AND PROJECT INTEGRATION}

With the passing of legislation designed to permanently cap and reduce mercury emissions from coal-fired utilities, it is more important than ever to develop and improve upon methods of controlling mercury emissions. One promising technique is carbon sorbent injection into the flue gas of the coal-fired power plant. Currently, this technology is very expensive as costly commercially activated carbons are used as sorbents. There is also a significant lack of understanding of the interaction between mercury vapor and the carbon sorbent, which adds to the difficulty of predicting the amount of sorbent needed for specific plant configurations.

Due to its inherent porosity and adsorption properties as well as on-site availability, carbons derived from gasifiers are potential mercury sorbent candidates. Furthermore, because of the increasing restricted use of landfilling, the coal industry is very interested in finding uses for these materials as an alternative to the current disposal practice.

The results of laboratory investigations and supporting technical assessments conducted under DOE Subcontract No. DE-FG26-03NT41795 are reported. This contract was with the University of Kentucky Research Foundation, which supports work with the University of Kentucky Center for Applied Energy Research and The Pennsylvania State University Energy Institute. The worked described was part of a project entitled "Advanced Gasification ByProduct Utilization". This work involved the development of technologies for the separation and characterization of coal gasification slags from operating gasification units, activation of these materials to increase mercury and nitrogen oxide capture efficiency, assessment of these materials as sorbents for mercury and nitrogen oxides, assessment of the potential for leaching of $\mathrm{Hg}$ captured by the carbons, analysis of the slags for cement applications, and characterization of these materials for use as polymer fillers.

The objectives of this collaborative effort between the University of Kentucky Center for Applied Energy Research (CAER), The Pennsylvania State University Energy Institute, and industry collaborators supplying gasifier char samples were to investigate the potential use of gasifier slag carbons as a source of low cost sorbent for $\mathrm{Hg}$ and $\mathrm{NO}_{\mathrm{X}}$ capture from combustion flue gas, concrete applications, polymer fillers and as a source of activated carbons. Primary objectives were to determine the relationship of surface area, pore size, pore size distribution, and mineral content on $\mathrm{Hg}$ storage of gasifier carbons and to define the site of $\mathrm{Hg}$ capture. The ability of gasifier slag carbon to capture $\mathrm{NO}_{\mathrm{X}}$ and the effect of $\mathrm{NO}_{\mathrm{X}}$ on $\mathrm{Hg}$ adsorption were goals. Secondary goals were the determination of the potential for use of the slags for cement and filler applications. Since gasifier chars have already gone through a devolatilization process in a reducing atmosphere in the gasifier, they only required to be activated to be used as activated carbons. Therefore, the principal objective of the work at PSU was to characterize and utilize gasification slag carbons for the production of activated carbons and other carbon fillers. Tests for the $\mathrm{Hg}$ and $\mathrm{NO}_{\mathrm{X}}$ adsorption potential of these activated gasifier carbons were performed at the CAER.

During the course of this project, gasifier slag samples chemically and physically characterized at UK were supplied to PSU who also characterized the samples for sorption characteristics and independently tested for $\mathrm{Hg}$-capture. At the CAER as-received slags were tested for $\mathrm{Hg}$ and $\mathrm{NO}_{\mathrm{X}}$ adsorption. The most promising of these were activated chemically. The PSU group applied thermal and steam activation to a representative group of the gasifier slag samples separated by particle sizes. The activated samples were tested at UK for Hg-sorption and $\mathrm{NO}_{\mathrm{X}}$ capture and the most promising $\mathrm{Hg}$ adsorbers were tested for $\mathrm{Hg}$ capture in a simulated flue gas. Both UK and PSU tested the use of the gasifier slag samples as fillers. The CAER analyzed the slags for possible use in cement applications. The division of tasks reduced overall 
overlap while still assuring redundant characterization and assessment to give an accurate view of the variability inherent to these types of materials. 


\section{TABLE OF CONTENTS}

\begin{tabular}{|c|c|}
\hline Disclaimer & 2 \\
\hline Executive Summary and Project Integration & 3 \\
\hline Table of Contents & 5 \\
\hline List of Figures & 6 \\
\hline List of Tables & 7 \\
\hline Work Performed at the University of Kentucky & 8 \\
\hline 1. Introduction & 9 \\
\hline 2. Experimental Methods & 10 \\
\hline 2.1. Optimization of Separation Process & 10 \\
\hline 2.2. Materials & 10 \\
\hline 2.3. Chemical and Physical Characterization of the Experimental Materials & 11 \\
\hline 2.4. Chemical Activation of Slags at the CAER & 12 \\
\hline 2.5. Mercury Adsorption Testing Apparatus and Procedures & 12 \\
\hline 2.5.1. Testing for Mercury Adsorption in Air & 12 \\
\hline 2.5.2. Testing for Mercury Adsorption in Simulated Flue Gas & 13 \\
\hline 2.6. $\mathrm{NO}_{\mathrm{X}}$ Adsorption Capacity Testing & 15 \\
\hline 2.7. Leaching of Slag with and without Mercury Exposure & 15 \\
\hline 2.8. Production of By-product Carbon Fillers & 15 \\
\hline 3. Results and Discussion & 16 \\
\hline 3.1. Materials Characterization & 16 \\
\hline 3.2. Mercury Adsorption Capacities & 21 \\
\hline 3.3. Mercury Adsorption on Activated Gasifier Slag Carbons & 23 \\
\hline 3.3.1. Thermal Activation & 23 \\
\hline 3.3.2. Chemical Activation & 23 \\
\hline 3.4. Mercury Binding Sites by XAFS Spectrometry & 24 \\
\hline 3.4.1. Mercury Determinations & 24 \\
\hline 3.4.2. Sulfur and Chlorine Determinations & 27 \\
\hline 3.5. $\mathrm{NO}_{\mathrm{X}}$ Adsorption Capacities for all As-received Gasifier Slags & 29 \\
\hline 3.6. $\mathrm{NO}_{\mathrm{X}}$ Adsorption Capacities of Activated Slag Samples & 29 \\
\hline 3.7. Adsorption of Mercury in a Simulated Flue Gas & 32 \\
\hline 3.8. Leachability of Mercury from Mercury Laden Gasifier Slag Carbons & 32 \\
\hline 3.9. Evaluation of Slag as a Supplementary Cementitious Material & 33 \\
\hline 3.10. Use of Gasifier Slag Carbon as Fillers & 34 \\
\hline 4. Conclusions & 38 \\
\hline 5. References & 41 \\
\hline
\end{tabular}




\section{LIST OF FIGURES}

\begin{tabular}{|c|c|c|}
\hline gure 2.1. & $\begin{array}{l}\text { Mercury adsorption testing apparatus with continuous } \mathrm{Hg} \text { vapor } \\
\text { monitoring }\end{array}$ & 13 \\
\hline Figure 2.2 & $\begin{array}{l}\text { Reactor system for the testing of gasifier by-products using simulated } \\
\text { flue gas }\end{array}$ & 14 \\
\hline Figure 3.1. & Elemental analysis of gasifier carbons & 17 \\
\hline Figure 3.2. & Anion analysis of gasifier carbons & 18 \\
\hline Figure 3.3 . & BET surface areas of all carbons st & 19 \\
\hline Figure 3.4. & Pore size distribution of gasifier slag carbons & 19 \\
\hline Figure 3.5. & BET surface areas for untreated and activated gasifie & 20 \\
\hline Figure 3.6. & Pore size distribution for untreated and activated gasifier $\mathrm{s}$ & 20 \\
\hline Figure 3.7. & Adsorption curve for $\mathrm{A} 3(-20+80)$ gasifier carbon & 21 \\
\hline igure 3.8 . & Adsorption curve for A2(-60) gasifier carbon & 22 \\
\hline igure 3.9. & Comparison of $\mathrm{Hg}$ capture potential of different gasifier carbons & 22 \\
\hline igure 3.10 . & $\begin{array}{l}\text { y adsorption capacities of untreated and activated gasifier slag } \\
\text {. }\end{array}$ & 23 \\
\hline gure 3.11 . & YANCS & 24 \\
\hline igure 3.12 . & $\begin{array}{l}\text { Definition and determination of the IPD parameter for Hg XANES } \\
\text { sprectra }\end{array}$ & 25 \\
\hline gure 3.13 . & EXAFS-RSF spectra for HgS and the two carbonaceous sorbents & 25 \\
\hline Figure 3.14 . & s fitting of the $\mathrm{k}^{3}$-weighted chi data region for & 26 \\
\hline gure 3.15 . & $f, Y A N D$ & 28 \\
\hline igure 3.16 . & Chlor & 28 \\
\hline igure 3.17 . & $\mathrm{NO}_{\mathrm{X}} \mathrm{a}$ & 30 \\
\hline Figure 3.18 . & $\begin{array}{l}\text { Correlation between } \mathrm{NO}_{\mathrm{X}} \text { adsorption capacity and } \mathrm{BET} \text { : } \\
\text { slags }\end{array}$ & 30 \\
\hline gure 3.19 . & $\begin{array}{l}\text { Correlation between } \mathrm{NO}_{\mathrm{x}} \text { adsorption capacity and micropore volume } \\
\text { of gasifier slags }\end{array}$ & 31 \\
\hline gure 3.20 . & $\mathrm{No}$ & 31 \\
\hline igure 3.21 . & $\mathrm{Hg}$ : & 32 \\
\hline igure 3.22 . & XRD & 34 \\
\hline igure 3.23 . & 3 in TPE-A & 35 \\
\hline Figure 3.24 . & $\begin{array}{l}\text { The strength and modulus for polypropylene with slag A3 were } \\
\text { measured with a simple tensile test }\end{array}$ & 35 \\
\hline 3.25 & $\begin{array}{l}\text { The measured strength and modulus values for TPE-A containing slag } \\
\text { A3 revealed that slag A3 did little to reinforce the TPE-A matrix }\end{array}$ & 36 \\
\hline Figure 3.2 & $\begin{array}{l}\text { ased with the addition of A3 but } \\
\text { th increasing sample temperature }\end{array}$ & 37 \\
\hline igure 3.2 & $\begin{array}{l}\text { The thermal diffusivity of TPE-A remained relatively unchanged with } \\
\text { the addition of A3 }\end{array}$ & 37 \\
\hline
\end{tabular}




\section{LIST OF TABLES}

\begin{tabular}{lll} 
Table 2.1. & Composite processing conditions & 16 \\
\hline Table 3.1. & XAFS IPD values and parameters from least-squares coordination & 27
\end{tabular} shell

Table 3.2. Chemical Composition of the +20 Mesh Slag Fractions 


\section{WORK PERFORMED AT THE UNIVERSITY OF KENTUCKY}




\section{Introduction}

A number of different processes for control of $\mathrm{Hg}$ emissions from electric power generation are being investigated which involve the use of a sorbent usually activated carbon as the primary Hg capture step (Bolger, et at.,2002; Clarke, L.B., et al. 1992; White, et. al., 1993). One problem associated with the use of activated carbon for $\mathrm{Hg}$ flue gas control is its high cost. Two approaches to reducing the cost of the utilization of a sorbent for $\mathrm{Hg}$ capture are the tailoring of activated carbons for $\mathrm{Hg}$ sorption to reduce the carbon to $\mathrm{Hg}$ ratio (Ghorishi, et. al., 2002; Lui, et. al., 2000) and the use of a low cost alternative sorbent (Chang, 1998; Serre and Silcox, 2000; Hwang and Li, 2000 patent). A potential source of less expensive Hg sorption material is the unburned carbon in gasifier char which is the focus of this study.

The CAER has studied the relationship between $\mathrm{Hg}$ capture and fly-ash characteristics and have demonstrated several fundamental relationships which should apply to gasifier chars. Highly significant correlations were found between the magnitude of $\mathrm{Hg}$ capture and $\mathrm{C}$ content for fly ashes, type of fly ash carbon, and the BET surface area (Hower et al., 1999, Hower et al., 2000; Sakulpitakphon et al. 2000; Mardon, S.M. and Hower, J.C., 2002). All these factors point to the possible utilization of high carbon gasifier char for $\mathrm{Hg}$ capture. Four gasifier slags were studied through the course of this project for their potential as Hg sorbents.

Mercury x-ray adsorption fine structure spectrometry (XAFS) has been used at the University of Kentucky as a direct, non-destructive probe of the local bonding and structure of $\mathrm{Hg}$ complexes involved in the adsorption of mercury species from combustion flue gases on sorbent materials (Huggins, et al, IAUS,1999; Huggins, et al, E\&F, 1999; Huggins, et al, Jpn. J. App. Phy, 1999; Huggins, 2003). Results indicated that the carbonaceous material in the fly ash is mostly responsible for the sorption of the mercury and provided evidence for different fractions in the carbon having different $\mathrm{Hg}$ sorption capabilities. The data also suggested sorption by aluminosilicates in the ash. Hg laden gasifier slag carbons produced during this work were submitted for XAFS to determine the binding sites of $\mathrm{Hg}$ on carbons.

The use of a carbon based sorbent for $\mathrm{Hg}$ capture from flue gas may have further benefits. Previous research at the CAER has shown that $\mathrm{NO}_{\mathrm{X}}$ is captured from simulated flue gas by activated carbons at conditions which appear compatible with $\mathrm{Hg}$ adsorption (Rubel, A. M., et al 1993; Rubel, A. M., et al, 1995; Rubel and Stencel, 1997). Therefore, injection of active gasifier slag carbon may have added benefit of decreased $\mathrm{NO}_{\mathrm{X}}$ emissions which was tested as part of this project.

The Hg adsorption capacities of four gasifier slag carbons and their separated particle size fractions were compared with respect to their $\mathrm{Hg}$ capture potential utilizing a $\mathrm{Hg}$ adsorption reactor at the CAER. These slag carbons were also studied for $\mathrm{NO}_{\mathrm{X}}$ adsorption alone and in conjunction with $\mathrm{Hg}$ capture. The data obtained provided some basic information as to the feasibility of utilizing gasifier chars for $\mathrm{Hg} / \mathrm{NO}_{\mathrm{X}}$ capture. Another important aspect of $\mathrm{Hg}$ adsorption on gasifier char which was addressed during this study was the leachability of the captured Hg.

Other potential uses of the gasifier slags were investigated. Since the gasifier slags from this study were to be submitted to extensive chemical analysis their potential for use in concrete applications was determined by the assessment of their composition in light of what is needed for pozzolanic activity. Preliminary experiments to determine the use of gasifier slag carbons as polymer fillers were performed in the hope of proving additional uses for gasifier slags. 


\section{Experimental Methods}

2.1. Optimization of Separation Process

At the time the original proposal was submitted, Charah, Inc. had installed and was operating a process to separate the gasification by-product produced at TECO's Polk Station. The processing plant produced three distinct products; coarse frit or slag $(>850 \mu \mathrm{m})$, an intermediate size high carbon fuel $(>75 \mu \mathrm{m},<850 \mu \mathrm{m})$ and fines $(<75 \mu \mathrm{m})$. The coarse frit and high carbon fuel were utilized or marketed while the fines were landfilled. The proposed research focused on evaluating the fines in order to identify potential upgrading alternatives that would enable beneficial reuse (Task 1 and 2).

Charah, Inc. successfully operated the processing plant to remediate the $140 \mathrm{~K}$ ton stockpile of slag stored at the Polk site along with daily production and completed the remediation project in late summer, 2004. At that time, a decision was made by TECO management to purchase the processing plant and assume operating responsibilities. Leading up to this change in ownership, several modifications were made to the processing plant in response to changes in gasifier operation. The construction of additional oxygen generating capacity at Polk now enables the carbon fuel as well as the fines to be re-circulated back to the gasifier as supplemental fuel. Thus the need to segregate the high carbon fuel from the fines was eliminated (requiring modification of Task 2 as proposed). Since the processing plant no longer produces a separate fines product, it was necessary to secure a similar material from an alternative source.

The Eastman Chemical gasifiers operated at the Kingsport, TN site are similar in design to the Chevron-Texaco gasifier operated at Polk. There are significant differences in the operation of the gasifiers at both sites since the primary objective at Polk Station is to produce electricity while the Eastman gasifiers produces syngas as a feedstock for chemical production.

At the Eastman site, molten slag is removed from the gasifier to a quench tank which is emptied at half-hour intervals through a lock hopper. The quenched slag drops into a settling tank and coarse material settles to the bottom where it is removed by a drag conveyor. Fine suspended solids $(<150 \mu \mathrm{m})$ are removed as a slurry, are flocculated, filtered on vacuum drum filters and disposed. Since the fines once produced at Polk Station are no longer available and there is no current or historical use for the filter cake produced as a by-product of gasification at Eastman, the Eastman substrate will be characterized and evaluated to identify any potential beneficial reuse options in accordance with the original objectives of the proposal.

2.2. Materials

Carbon rich slag samples were obtained from two gasification plants, $\mathrm{A}$ and $\mathrm{B}$, for this study. Both plants feed coal but plant A sometimes feeds some petroleum coke and occasionally all petcoke. An insufficient number of slags were acquired for this study to determine the effect of the petcoke addition. The slags were separated according to different particle size fractions for testing for their $\mathrm{Hg}$ and $\mathrm{NO}_{\mathrm{X}}$ adsorption capability. The samples were tested as received and activated using thermal and steam activation procedures. Samples were taken from plant A on two separate occasions and once from plant B. The samples were labeled according to the plant from which they were received followed by their particle size distribution. The sample identification were as follows: A1(-20+80), A2(-20+80), A2(-80), B(-20+80), B(-80), B(soot), and $\mathrm{B}(\mathrm{fcake}=$ filter cake $)$. A commercial activated carbon, HGR, which was developed for high $\mathrm{Hg}$ adsorption was used for comparison.

Portions of the following separated slag samples were sent to the Energy Institute (EI), The Pennsylvania State University, for activation by established thermal and steam procedures: $\mathrm{A} 1(-20+80), \mathrm{B}(-20+80), \mathrm{B}(-80), \mathrm{B}($ soot $)$, and $\mathrm{B}(+20)$. The activated samples were tested for $\mathrm{Hg}$ and $\mathrm{NO}_{\mathrm{X}}$ adsorption at the CAER. 
Select samples of the slag fractions were also tested for $\mathrm{Hg}$ adsorption in a simulated flue gas. These samples were A1(-20+80), A2(-20+80), B(-20+80). HGR was also tested for comparison under the same conditions described below.

2.3. Chemical and physical characterization of the experimental materials

All as received gasifier carbons were submitted to chemical analysis for ultimate, proximate, forms of sulfur, and anions analyses by standard procedures. Surface areas were analyzed according to the Brunauer, Emmett, and Teller (BET) method (Brunauer, et. al., 1938) and pore size distribution by density functional theory (DFT) using Micromeritics DFT Plus software (Dreizler, et. al., 1998). BET surface areas and pore size distributions were also done on all activated gasifier carbons produce at EI. By IUPAC standard, pore sizes are defined according to width as: micropores $(<2 \mathrm{~nm})$, mesopores $(2$ to $50 \mathrm{~nm})$, and macropores $(>50 \mathrm{~nm})$.

In order to define the relationship between $\mathrm{Hg}$ and adsorptions sites on the gasifier carbons, three samples of slags were analyzed by $\mathrm{Hg}$ X-ray absorption fine structure (XAFS) spectroscopy. These samples are as follows:

Sample A1(run 20+21): Carbonaceous solid with high loading of $\mathrm{Hg}$

Sample A2(run 22): Carbonaceous solid with light loading of $\mathrm{Hg}$

Sample A1(run 26): Control sample with no Hg loading

XAFS data were also obtained on the $\mathrm{S}$ and $\mathrm{Cl}$ species present in these samples.

Samples were subjected to mercury $\mathrm{L}_{\mathrm{III}}$-edge XAFS spectroscopy at both beam-line X$18 \mathrm{~B}$ at the National Synchrotron Light Source (NSLS), Brookhaven National Laboratory, NY, and beam-line 11-2 at the Stanford Synchrotron Radiation Laboratory (SSRL), Stanford University, CA. The data obtained at the latter facility were significantly better in terms resolution and signal/noise ratio and the results described herein are based only on the data obtained at SSRL, although there was good agreement between the data obtained at both laboratories. Sulfur and chlorine K-edge XAFS data are from beam-line X-19A at NSLS.

Mercury $\mathrm{L}_{\mathrm{II}}$-edge XAFS spectra were obtained at SSRL using a silicon (220) double crystal monochromator over the energy range from 12,200 to as much as $13,000 \mathrm{eV}$. The spectra were acquired in fluorescent geometry using a high signal/noise throughput, 30-element Ge detector (Cramer et al., 1988), Soller slits and a 6u Ga filter (Stern and Heald, 1979), and multiple scanning. The net effect of these experimental arrangements was the collection of superior quality XAFS spectra, despite the relatively low $\mathrm{Hg}$ content in sample (22). Samples of $\mathrm{HgS}$ (metacinnabar), yellow $\mathrm{HgO}$, red $\mathrm{HgO}, \mathrm{HgNO}_{3}$, and $\mathrm{Hg}$ metal were also collected at the same session under similar conditions. However, a standard transmission detector and Lytle fluorescence detector (Lytle et al., 1984) were used for the XAFS measurements on the standard $\mathrm{Hg}$ compounds. Primary calibration of the energy scale was achieved by running the spectrum of metallic mercury in a simultaneous transmission experiment behind the fluorescent experiment on the carbonaceous solids. The major peak in the derivative $\mathrm{Hg} \mathrm{L}_{\mathrm{III}}$-edge XANES spectrum of elemental $\mathrm{Hg}$ was assumed to occur at $12,284 \mathrm{eV}$.

Sulfur and chlorine K-edge XAFS spectra were obtained at NSLS using a silicon (111) double crystal monochromator over the energy range from $2,400 \mathrm{eV}$ to $2,900 \mathrm{eV}$. This region included the absorption edges for both $\mathrm{S}$ and $\mathrm{Cl}$ at 2,472 eV and 2,825 eV, respectively. The spectra were acquired in a modified Lytle fluorescent detector using a PIPS detector to measure the fluorescent $\mathrm{X}$ radiation. Helium was employed as the sample chamber flush gas. Primary calibration of the energy scale for both $\mathrm{S}$ and $\mathrm{Cl} \mathrm{K}$-edges was achieved by means of a pellet of SOMAR mix containing $2 \mathrm{wt} \%$ of elemental sulfur. The zero-point of energy was assumed to occur at the main peak position in the sulfur K-edge XAFS spectrum of elemental sulfur. Unfortunately, no $\mathrm{NaCl}$ was available to calibrate the position of the $\mathrm{Cl} \mathrm{K}$-edge precisely so the elemental sulfur calibration was also used to calibrate the position of the $\mathrm{Cl} \mathrm{K}$-edge. 
Data analysis followed conventional practice (Eisenberger and Kincaid, 1978, Lee et al., 1981, Brown et al., 1988, Koningsberger and Prins, 1988) in that the energy scales of the carbonaceous sorbents were first adjusted according to the $\mathrm{Hg} \mathrm{L}_{\mathrm{III}}$-edge position in elemental $\mathrm{Hg}$. Then the spectral data were normalized to unit edge-step and separate X-ray absorption nearedge structure (XANES) and extended X-ray absorption fine structure (EXAFS) regions were obtained from the normalized XAFS data. The XANES region is used as a fingerprint for the $\mathrm{Hg}$ species under investigation. The only manipulation done on this region of the spectrum was to smooth the data and determine the first derivative of the spectrum in order to estimate the inflection point difference (IPD) parameter (Huggins, Yap, N., and Huffman, G. P., 1999). As discussed in detail elsewhere (Huggins et al., 1999, 2003), this parameter is a direct probe of the local bonding of the ligand atoms to the central $\mathrm{Hg}^{2+}$ ion and its value often reflects the element to which the $\mathrm{Hg}^{2+}$ is bound. The EXAFS region was converted from a real space to a reciprocal space (k-space) representation to yield the chi vs. $\mathrm{k}$ spectrum. The chi spectrum was then weighted by $\mathrm{k}^{3}$ and subjected to a Fourier transform to yield a radial structure function (RSF), which is basically a one-dimensional representation of the structure local to the X-ray absorbing $\mathrm{Hg}$ atom.

Data analysis for sulfur and chlorine was limited to generating the XANES spectra for both elements as the EXAFS region could not be successfully processed due to the limited energy range afforded to the two elements.

2.4. Chemical Activation of slags at the CAER

Slag char samples were mixed with $\mathrm{KOH}$ at ratios ranging 1:1 to $3: 1(\mathrm{KOH}$ : carbon) by weight. Sufficient water was added to form a paste-like slurry, and held at room temperature for 1 hour. The slurry was transferred to an air-tight furnace heated to $110{ }^{\circ} \mathrm{C}$ and held at temperature under flowing nitrogen for 12 hours to insure $\mathrm{KOH}$ impregnation. Subsequently, the samples were heat-treated to a final temperature ranging from 875 to $1000{ }^{\circ} \mathrm{C}$ with a dwell time of 60 minutes. After cooling in nitrogen, the material was Soxhlet extracted with water ( $5 \%$ $\mathrm{HCl})$ to a final $\mathrm{pH}$ of 7 to insure removal of residual $\mathrm{K}$ and then dried in a vacuum oven. 2.5. $\mathrm{Hg}$ adsorption testing apparatus and procedures

2.5.1. Testing for $\mathrm{Hg}$ adsorption in air. A Hg adsorption reactor (Figure 2.1) was used to test as-received and activated gasifier carbons in air. The device was constructed completely of Teflon tubing and fittings. The major components comprise a $\mathrm{Hg}$ permeation tube (VICI Metronics) maintained at a constant temperature in a hot oil bath, a 3/8-inch Teflon fixed bed reactor with quick connect fittings, a VM3000 continuous Hg vapor monitor, and a computer driven data acquisition system. The entire device is heat traced and insulated to maintain a constant temperature throughout the system. Adsorption temperatures up to $200^{\circ} \mathrm{C}$ are possible. The concentration of $\mathrm{Hg}^{0}$ vapor can be regulated by the temperature of the permeation tube and dilution of the $\mathrm{Hg}^{0}$ vapor with a purge gas. The system can be operated continuously 24 hours a day for multiple days. The entire system was tested for $\mathrm{Hg}$ retention and was found inert to $\mathrm{Hg}$ adsorption.

The device was operated under the following conditions to compare the adsorption capacities of the untreated gasifier carbons: a $\mathrm{Hg}$ concentration of $200 \mathrm{ug} / \mathrm{m}^{3}$ at $50^{\circ} \mathrm{C}$; $\mathrm{UPH}$ air as the carrier gas at $75 \mathrm{ml} / \mathrm{min}$ metered at room temperature and pressure; and $100 \mathrm{mg}$ of gasifer slag carbon. The $\mathrm{Hg}$ concentration in the gas exiting the fixed bed reactor was continuously monitored with data acquired by computer every second. Adsorption capacities were compared as the amount of $\mathrm{Hg}$ captured on the char at $10 \%$ breakthrough from the reactor of the $\mathrm{Hg}$ concentration in the adsorption gas. The data was normalized for the weight of the bed. $\mathrm{Hg}$ laden sorbent were analyzed for $\mathrm{Hg}$ concentration by x-ray fluorescence (XRF) analysis and standard procedures. 


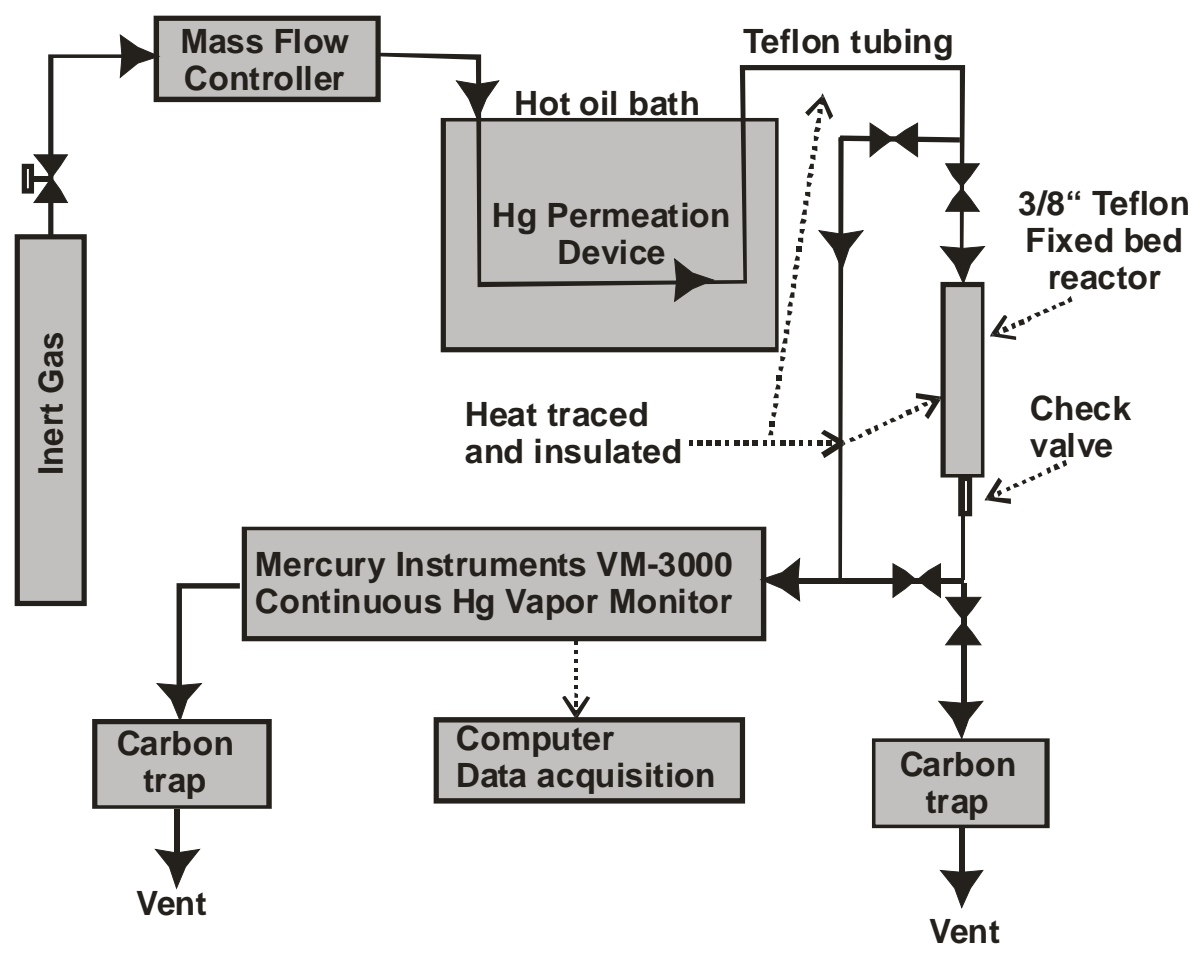

Figure 2.1. Mercury adsorption testing apparatus with continuous $\mathrm{Hg}$ vapor monitoring

Samples for XAFS were generated under slightly different conditions necessitated by the requirement of larger samples for XAFS analysis. The reactor was loaded with $500 \mathrm{mg}$ carbon and $\mathrm{Hg}$ vapor concentration was increased to $300 \mathrm{ug} / \mathrm{m}^{3}$ in order to decrease the adsorption time for breakthrough with the larger sample size when gasifier slag carbons with high $\mathrm{Hg}$ adsorption capacities were used. Otherwise conditions were the same.

Testing of activated gasifer slag samples were done under slightly different condition since the length of time required for breakthrough of the good $\mathrm{Hg}$ sorbent would have been prohibitive considering the number of activated samples tested. All samples were exposed to Hg-laden gas for 48 hour adsorption time which was experimentally determined as optimum and would keep high mercury values for good slag carbons within reasonable limits and yet enough mercury capture by poor adsorbing slags carbons for accurate analyses by XRF. The purge gas to the Hg permeation cell was UHP air at a flow rate of $100 \mathrm{ml} / \mathrm{m}$ metered at room temperature and pressure. $\mathrm{The} \mathrm{Hg}^{0}$ concentration in the gas stream was $200 \mathrm{ug} / \mathrm{m}^{3}$. Slag loaded into the reactor was $200 \mathrm{mg}$. The entire system was maintained at $60^{\circ} \mathrm{C}$.

2.5.2. Testing for mercury adsorption in simulated flue gas. The mercury adsorption testing apparatus was significantly modified for the determination of mercury adsorption under simulated flue gas conditions (Figure 2.2). The entire apparatus was constructed of materials which do not adsorb mercury, mainly Teflon tubing and fittings. The system had three mass flow controllers to blend air with gas mixtures of $\mathrm{NO}$ in $\mathrm{N}_{2}$ and $\mathrm{SO}_{2}$ in $\mathrm{N}_{2}$. The three gases were blended before a mercury permeation device which added mercury vapor to the simulated flue gas before passing through either an adsorption reactor or a reactor bypass tube. Two mercury vapor analyzers were used to monitor $\mathrm{Hg}^{0}$ concentrations leaving the mercury permeation device and to measure the mercury concentration leaving the fixed bed adsorption reactor containing the test material. The two instruments were a Jerome $431 \mathrm{X} \mathrm{Hg}$ vapor analyzer and a Mercury Instruments VM 3000 continuous Hg vapor analyzer. The Jerome 431X requires the collection of a gas sample in a gas collection bag before analysis. This instrument was used to measure the 


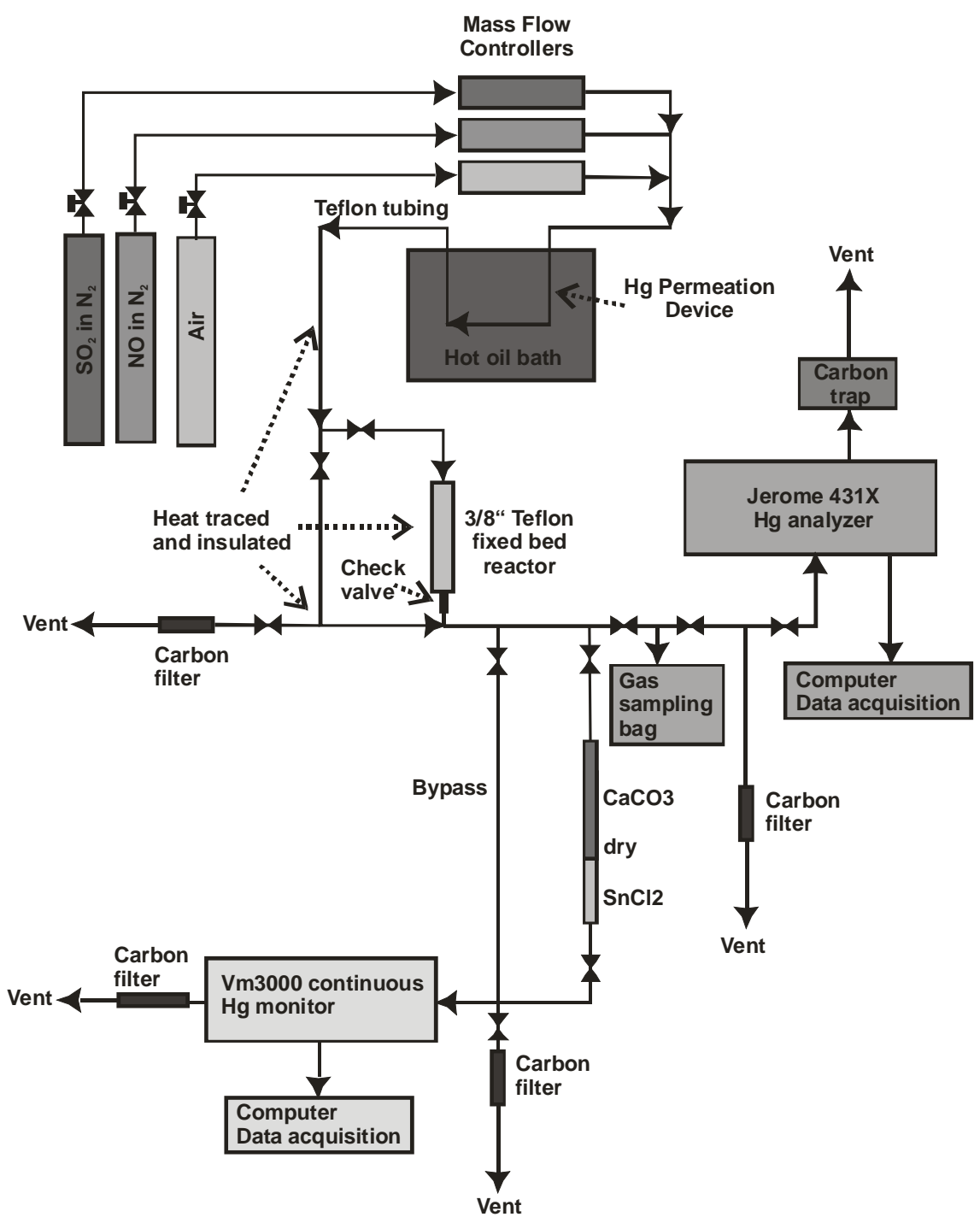

Figure 2.2. Reactor system for the testing of gasifier by-products using simulated flue gas.

concentration of $\mathrm{Hg}$ vapor at the beginning and end of each run as a check of the mercury concentration detected by the second instrument, the Mercury Instruments VM 3000, which was used to continuously monitor mercury vapor concentrations leaving the adsorption reactor or the bypass tube. This instrument detects $\mathrm{Hg}^{0}$ concentrations by cold vapor atomic adsorption spectrometry (CVAAS). $\mathrm{SO}_{2}$ interferes with this method of detection and both analyzers only detect $\mathrm{Hg}^{0}$. In the presence of reactive gases such as $\mathrm{NO}$ and $\mathrm{SO}_{2}$ especially in the presence of carbon, a possible catalyst, there is the possibility of conversion of $\mathrm{Hg}^{0}$ to $\mathrm{Hg}^{+2}$, which would not be detected. In order to address the limitations of CVAAS (interference by $\mathrm{SO}_{2}$ and $\mathrm{Hg}^{+2}$ in the gas), the gases from the reactor were passed through a column packed first with finely crushed $\mathrm{CaCO}_{3}$ followed by fine particles of $\mathrm{SnCl}_{2}$ (Figure 2.2). The $\mathrm{CaCO}_{3}$ was used to scrub the $\mathrm{SO}_{2}$ from the gas stream (Reaction 2.1 and 2.2) and the $\mathrm{SnCl}_{2}$ was used to reduce $\mathrm{Hg}^{+2}$ to $\mathrm{Hg}^{0}$ according to Reaction 2.3.

Reaction 2.1: $\mathrm{SO}_{2}+\mathrm{CaCO}_{3} \rightarrow \mathrm{CaSO}_{3}+\mathrm{CO}_{2}$

Reaction 2.2: $\mathrm{SO}_{2}+\mathrm{CaCO}_{3}+1 / 2 \mathrm{O}_{2}+\mathrm{H}_{2} \mathrm{O} \rightarrow \mathrm{CaSO}_{4} \cdot 2 \mathrm{H}_{2} \mathrm{O}+\mathrm{CO}_{2}$

Reaction 2.3: $\mathrm{Sn}^{+2} \mathrm{Cl}_{2}^{-}+\mathrm{Hg}^{+2} \rightarrow \mathrm{Sn}^{+4} \mathrm{Cl}_{4}^{-}+\mathrm{Hg}^{0}$ 
The simulated flue gas testing procedures for testing the gasifier carbons using the reactor system just described were establish to allow a sufficiently large sample of gasifier slag to be run to a comparable end point in a reasonable amount of time. The constraints of the time factor were to capture sufficient mercury in the sorbent to get accurate and reproducible numbers for mercury in the solids by XRF. A 48 hour adsorption time was experimentally determined as optimum for this study. This time would keep high mercury values for good sorbents within reasonable limits and yet enough mercury capture by poor sorbents for accurate analyses. The composition of the simulated flue gas was $125 \mathrm{ppm} \mathrm{NO}, 125 \mathrm{ppm} \mathrm{SO}_{2}$, with a balance of air. The $\mathrm{Hg}^{0}$ concentration in the gas stream was $200 \mathrm{ug} / \mathrm{m}^{3}$. The gas flow rate through the system was $100 \mathrm{ml} / \mathrm{m}$ metered at room temperature and pressure. Sorbent load was $200 \mathrm{mg}$. The entire system was maintained at $60^{\circ} \mathrm{C}$.

2.6. NOX adsorption capacity testing

A Netzsch STA449C thermal analyzer coupled to a Balzer Thermostar quadrupole mass spectrometer was used to measure the $\mathrm{NO}_{\mathrm{X}}$ adsorption capacity of the experimental materials. Procedures for the acquisition of $\mathrm{NO}_{\mathrm{X}}$ adsorption isotherms by thermal analysis - mass spectrometry (TG-MS) in simulated flue gas were previously developed at the CAER (Rubel et al., 1993). These procedures were modified for this work. In earlier work $\mathrm{CO}_{2}$ and $\mathrm{SO}_{2}$ were included in the simulated flue gas and shown to not significantly affect $\mathrm{NO}_{\mathrm{X}}$ adsorption on activated carbons. Therefore, these gases were not used in this study to simplify the test matrix. The adsorption gas mixture was $0.5 \% \mathrm{NO}, 5 \% \mathrm{O}_{2}$ with a balance of $\mathrm{He}$ and the adsorption temperature was $30^{\circ} \mathrm{C}$. All test materials were pre-conditioned by heating to the maximum desorption temperature, $250^{\circ} \mathrm{C}$, to be used after the adsorption step during capacity testing. The sorbent was then cooled to $30^{\circ} \mathrm{C}$ before exposure to the adsorption gas mixture. $\mathrm{NO}_{\mathrm{X}}$ adsorption capacity was considered to be the weight gained by the sample which could be attributed to $\mathrm{NO}_{\mathrm{X}}$ uptake by the sorbent using the established TG-MS procedures (Rubel, et. al., 1993).

2.7. Leaching of slag with and without $\mathrm{Hg}$ exposure

The Polk slags carbons were leached in distilled and deionized water $(>17$ mohms $)$ for 48 hours on an end-over-end TCLP tumbler. The baseline sample contained $50.0 \mathrm{~g}$ of slag in $228.9 \mathrm{~g}$ of D\&D water (4.6:1 liquid:solid ratio). The Hg-loaded samples consisted of $50.0 \mathrm{~g}$ of slag in $218.0 \mathrm{~g}$ of D\&D water (4.4:1 liquid:solid ratio). The liquid:solid ratios were selected to provide minimum sample volumes for cold vapor AA analyses without creating extremely dilute and perhaps unrealistic leachates (Kosson et al., 2002). The leachates should simulate leaching by clean rainwater through a slag pile.

After 48 hours, the leachates were filtered at 0.45 microns. The $\mathrm{pH}$ values of the filtered baseline and $\mathrm{Hg}$-loaded leachates were 3.86 and 3.84, respectively. Following procedures in Keeler and Landis, 1994 and the recommended operating procedures for the Ohio Lumex Co. $\mathrm{RA}-915+$ Zeeman $\mathrm{Hg}$ analyzer, the leachates were digested with $\mathrm{BrCl}$ overnight and excess $\mathrm{BrCl}$ was eliminated with hydroxylamine hydroxide before analysis with the cold vapor AA RA-915+ $\mathrm{Hg}$ analyzer using a stannous chloride reductant.

2.8. Production of by-product carbon fillers

All composite materials were prepared with a Haake Polylab shear mixing system. Three concentrations $(10,20,40 \%$ by weight) of Slag A3 were dispersed into the molten phase of two provided polymers: molding grade polypropylene and Thermoplastic Elastomer A (TPE-A; Kuraray, INC). Both polymers were also processed under similar conditions without additives. Each blending process began with $50 \mathrm{~g}$ of the raw materials with $\sim 90 \%$ of the original mass recovered from the mixing bowl. The mixing conditions are presented in Table 2.1. The mixing time of 43 minutes is broken down into three parts: 1) the polymer melts in the first three 
minutes, 2) the filler is added to the mixer at the three minute mark, and 3) mixing of the two phases commences for the remaining forty minutes. Roller rotors were used for all mixing runs.

Table 2.1. Composite processing conditions.

\begin{tabular}{||l|l|l|l|l||}
\hline & ATMOSPHERE & TEMPERATURE & $\begin{array}{l}\text { ROTOR } \\
\text { SPEED }\end{array}$ & TIME \\
\hline Polypropylene & Air & $180^{\circ} \mathrm{C}$ & $20 \mathrm{rpm}$ & $43 \mathrm{~min}$ \\
\hline $\begin{array}{l}\text { Thermoplastic } \\
\text { Elastomer A }\end{array}$ & Air & $180^{\circ} \mathrm{C}$ & $20 \mathrm{rpm}$ & $43 \mathrm{~min}$ \\
\hline
\end{tabular}

After recovery and cooling, each blended material was further processed by chopping it into small pellets with a low rpm chipper. The pellets were hot $\left(180^{\circ} \mathrm{C}\right)$ pressed into films of uniform thickness $(0.4 \mathrm{~mm}$ for polypropylene; $1.45 \mathrm{~mm}$ for TPE-A) using a Carver 10-ton press with heated platens. The electrical conductivity of each blend was measured using a ProStat Surface Resistance \& Resistivity Indicator (PSI-870). The copper contacts of the resistivity meter were aligned in intimate contact with the composite films. The surface resistivity of the film was measured and recorded. This data was presented as the log of the surface resistivity per unit square. If the film was insulating it was assigned a log value of 15.

All mechanical testing was performed on a MTS QTest 10 materials testing system. Dog bone shaped specimens were punched out of the uniform thickness films of each blend with a die constructed to match the specifications of ASTM D638. Prior to testing, the width and thickness of each specimen's gage section were recorded. Each specimen was loaded until failure at a crosshead speed of $5 \mathrm{~mm} / \mathrm{min}$. The TestWorks 4 software package was used to calculate peak tensile stress, yield stress, and modulus.

The thermal diffusivity of each composite material was measured with a Netzsch Laser Flash Analyzer (LFA). Cylindrical coupons (12.5 mm diameter; $1-1.3 \mathrm{~mm}$ thickness) were processed from each chopped material. A laser pulse (405 Volts; 1 ms pulse width) was emitted onto the bottom surface of the sample. The thermal diffusivity was measured as the temperature of the top surface of the sample changes. Thermal diffusivity was quantified as area per unit time $\left(\mathrm{mm}^{2} / \mathrm{sec}\right)$.

\section{Results and Discussion}

\subsection{Materials characterization}

Four different gasifier slag carbons, labeled A1, A2, A3 and B, were studied. Two of the gasifier slag carbons were separated into different particle size fractions. The particle size fractions were as follows: A1 (-20+80), A2 (-20+80), A2 (-60), A2 (-80), A3(-20+80), B(-20 $+80)$, B (soot), B (-80). The elemental analyses for all the gasifier carbons are shown in Figure 3.1. Carbon content which is thought to be important in $\mathrm{Hg}$ capture varied from 7 to $77 \%$. The $\% \mathrm{O}$ by difference was high in samples $\mathrm{A} 1(-20+80), \mathrm{A} 3(-20+80)$ and $\mathrm{B}$ (soot). Figure 3.2 gives the anions present in the gasifier samples. A1, A2 (-80) fraction, and A3 were high in both $\mathrm{Cl}^{-}$ and $\mathrm{F}^{-}$compared to the other samples. $\mathrm{Br}^{-}$and $\mathrm{PO}_{4}{ }^{-2}$ were 4 to 5 times higher in slags $\mathrm{A} 1$ and A3. $\mathrm{Cl}^{-}$and $\mathrm{Br}^{-}$have been implicated in good $\mathrm{Hg}$ capture whereas A1, A2 (-80), A3, and B (-20 $+80)$ were high in $\mathrm{SO}_{4}{ }^{-2}$, another anion which has been associated with good $\mathrm{Hg}$ adsorption. Most of the $\mathrm{S}$ in the samples was associated with $\mathrm{SO}_{4}{ }^{-2}$.

All slag samples were subjected to BET surface area shown in Figure 3.3. Gasifier slag carbon, A2, had lower surface area than carbons A1, A3, and B. A2 and B had lower total pore volume than $\mathrm{A} 1$ and $\mathrm{A} 3$ which had much larger meso and macropore volumes than $\mathrm{A} 2$ and $\mathrm{B}$ 
(Figure 3.4). These data will be discussed in relation to the $\mathrm{Hg}$ and $\mathrm{NO}_{\mathrm{X}}$ adsorption capacities of the carbons. BET SA and pore size distributions were also performed on all activated slag samples from EI. The results are given in Figures 3.5 and 3.6. The B $(-20+80), A 1(-20+80)$, and B (+20) slag samples had increases in SA of $2.5,2$, and 1.7 times their untreated samples respectively. The B (soot) and the B (-80) had very little increase in SA under the conditions studied. There were no significant changes in SA due to increased time or steam. The increase in SA was primarily due to significant increases in mesopore volumes. However, micropore volumes also increased in $\mathrm{B}(-20+80)$ and $\mathrm{A} 1(-20+80)$.

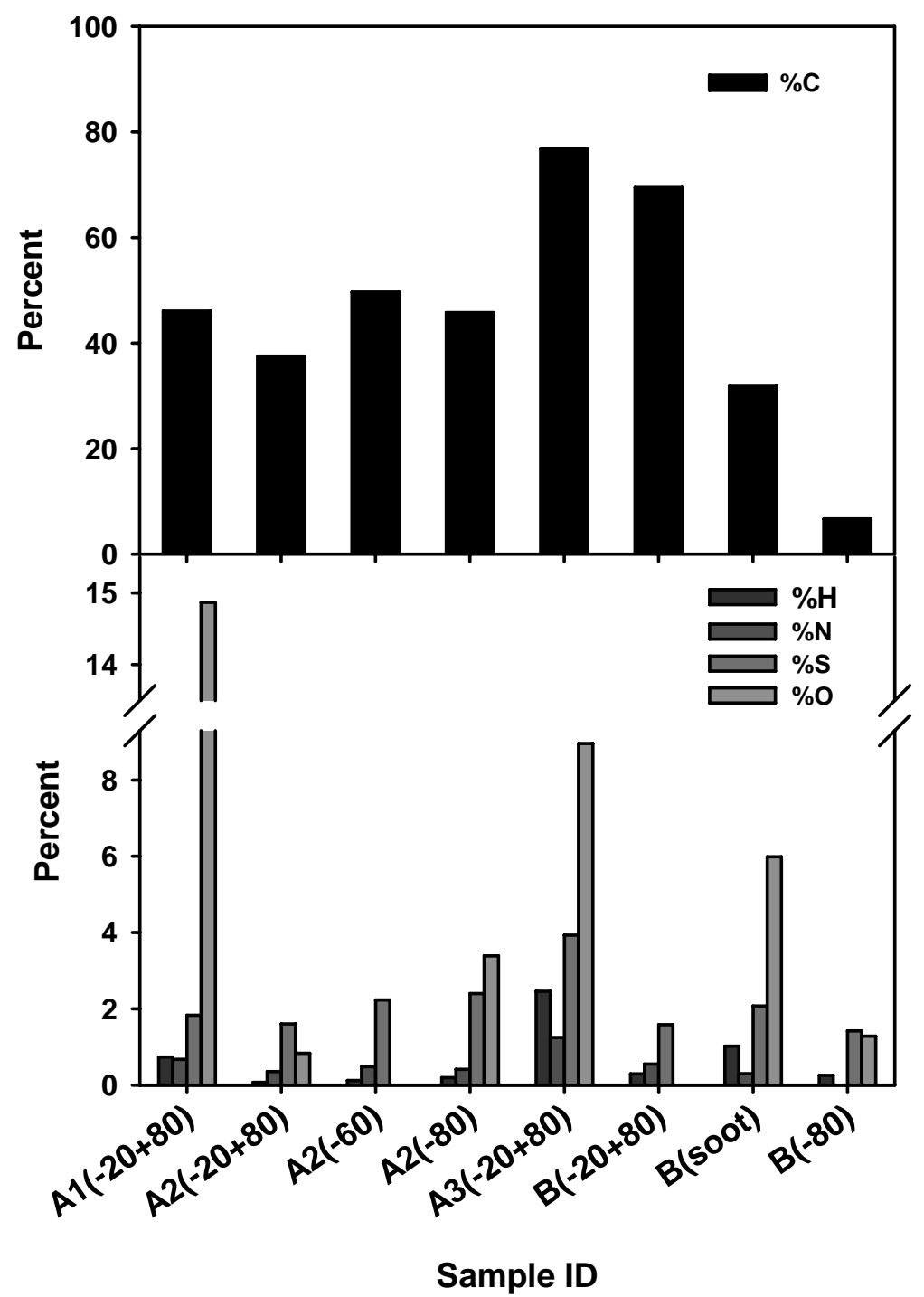

Figure 3.1. Elemental analysis of gasifier carbons. 


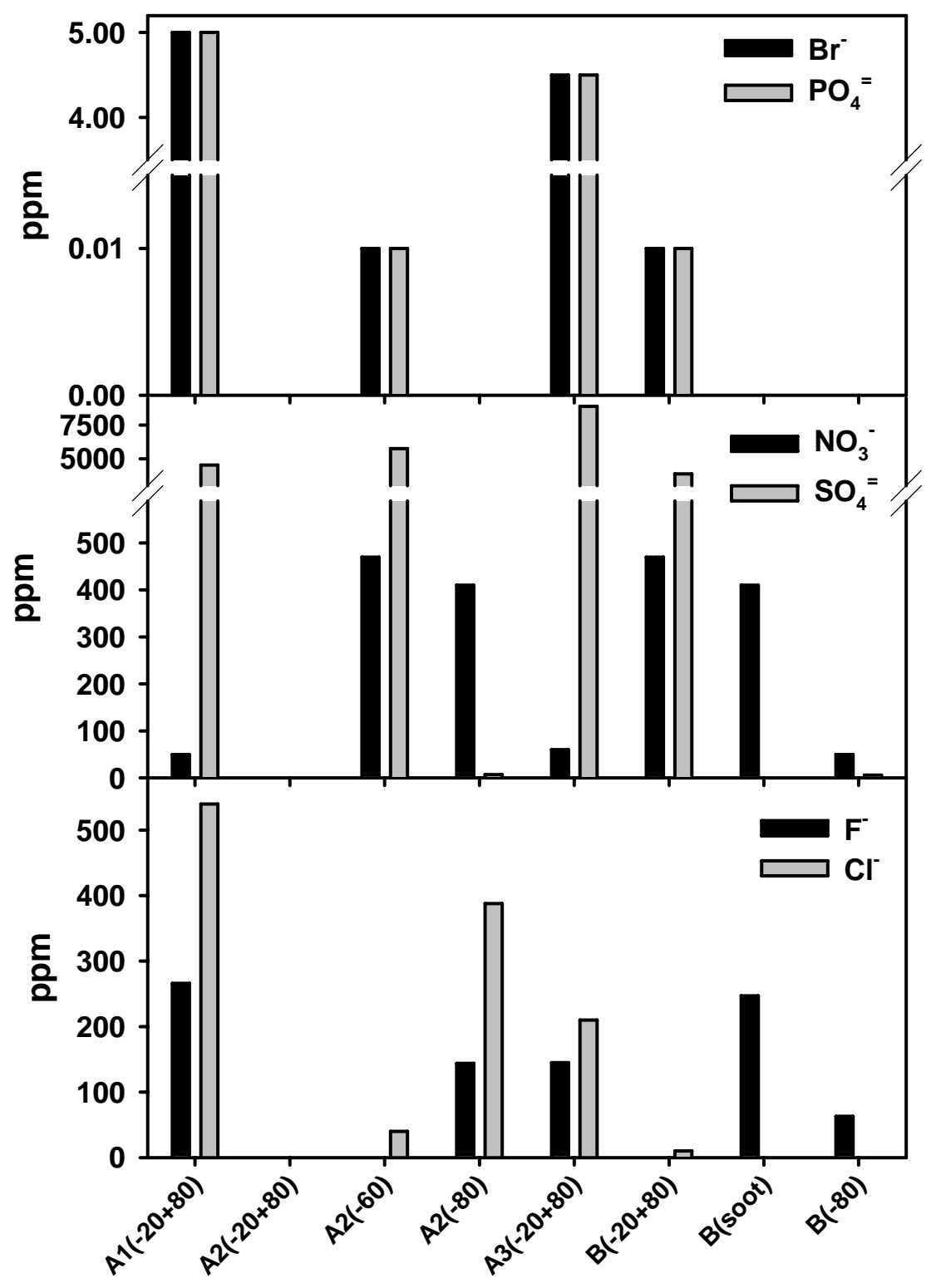

Sample ID

Figure 3.2. Anion analysis of gasifier carbons. 


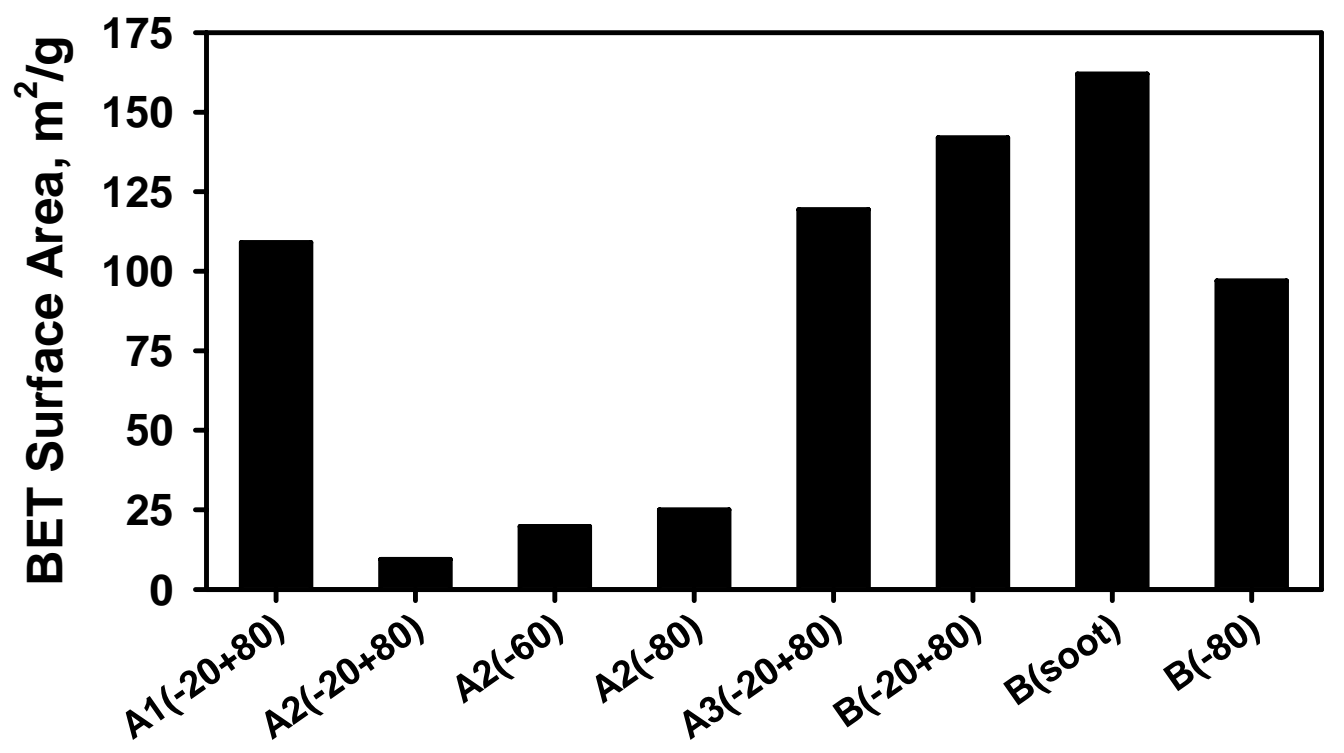

Sample ID

Figure 3.3. BET surface areas of all carbons studied.

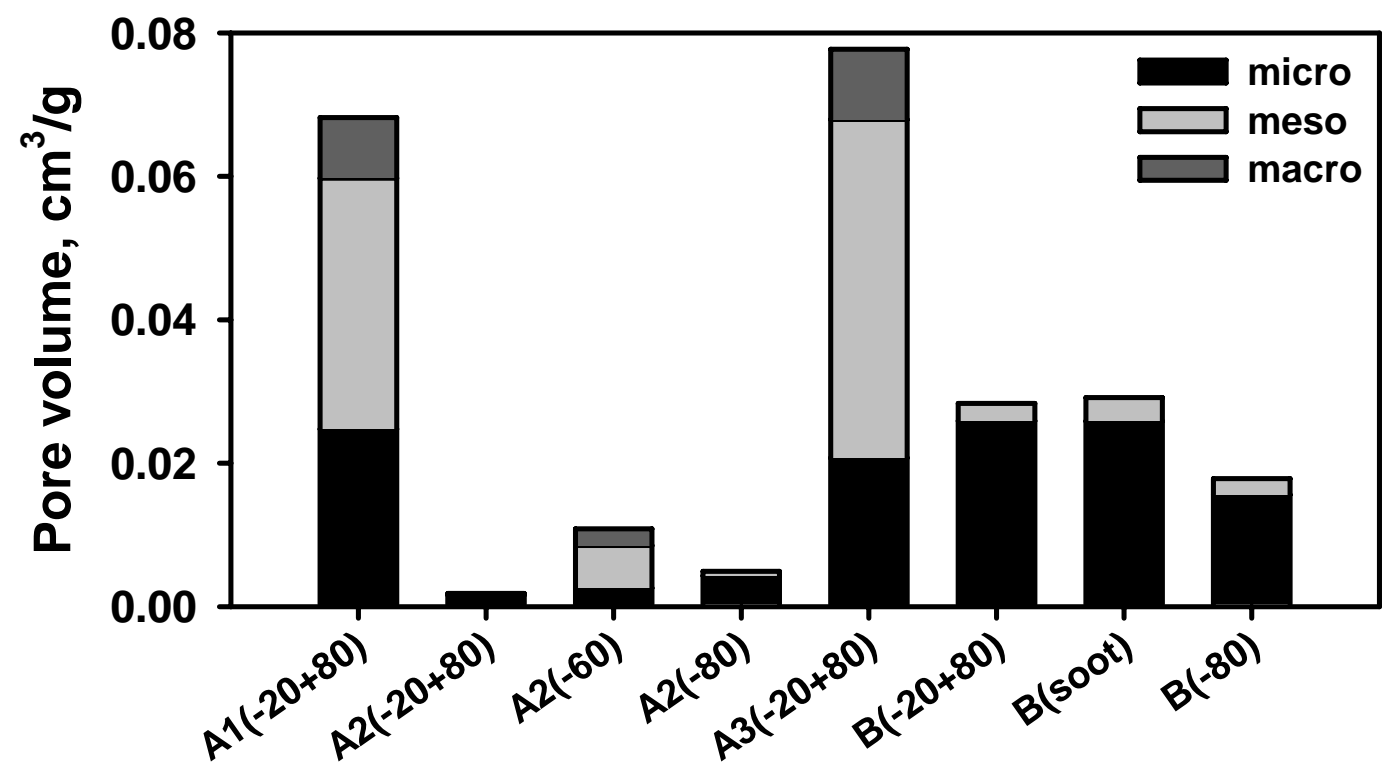

\section{Sample ID}

Figure 3.4. Pore size distribution of gasifier slag carbons. 


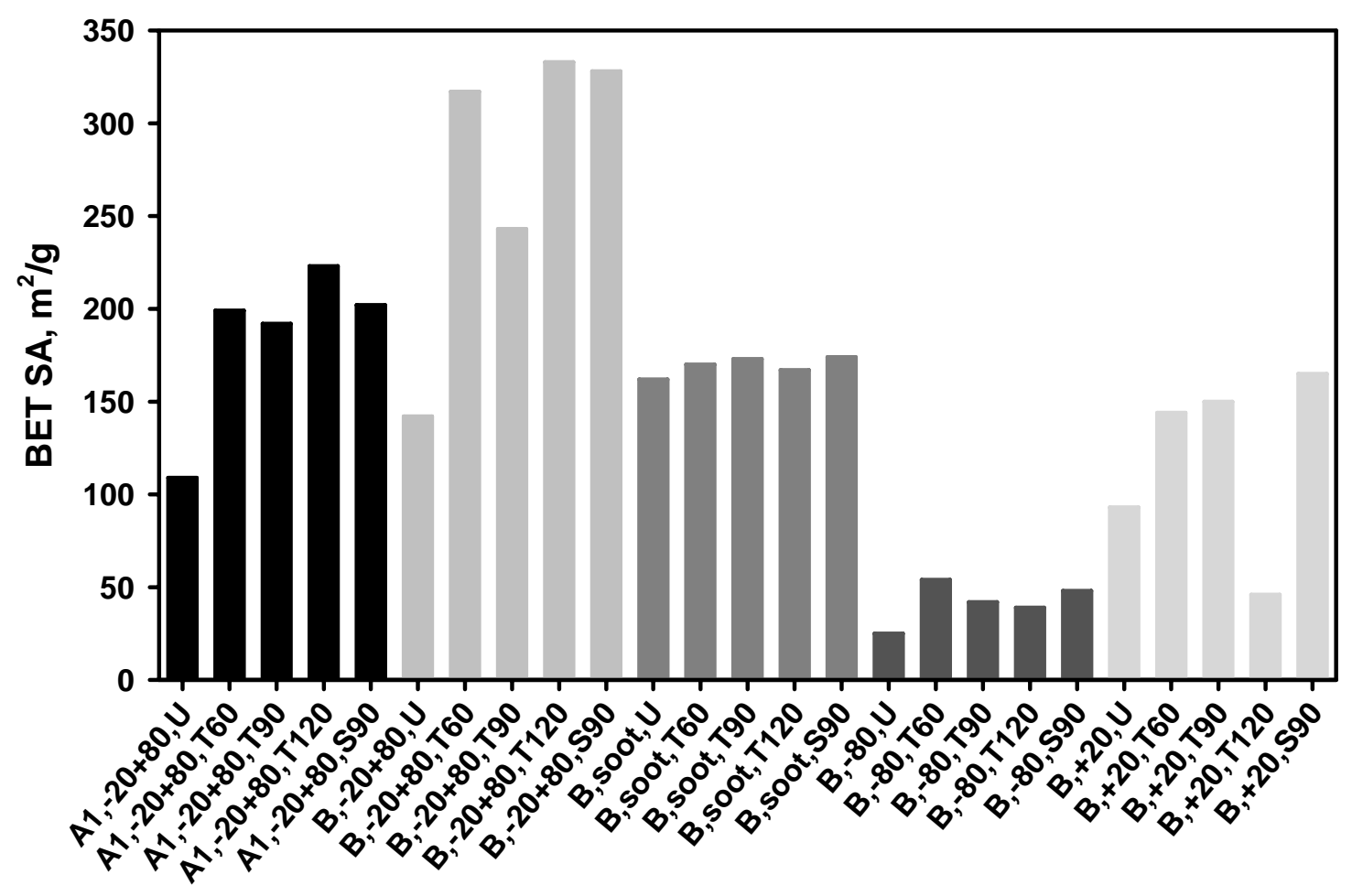

Sample I.D.

Figure 3.5. BET surface areas for untreated and activated gasifier slag carbons.

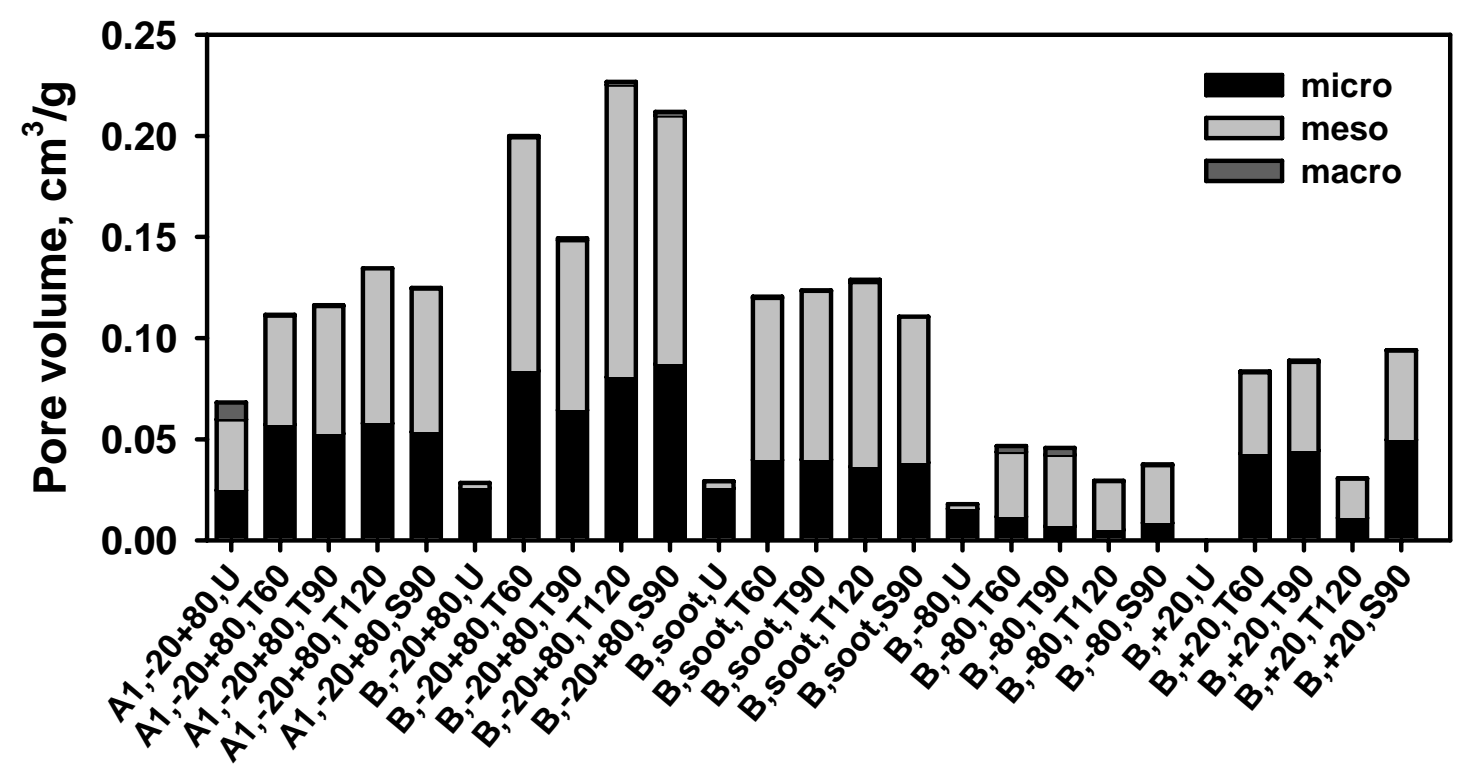

Sample ID

Figure 3.6. Pore size distribution for untreated and activated gasifier slag samples. 


\subsection{Hg adsorption capacities}

$\mathrm{Hg}$ adsorption capacities were compared at $10 \%$ breakthrough $\left(20 \mathrm{ug} / \mathrm{m}^{3}\right)$ of the concentration of the $\mathrm{Hg}$ in the gas leaving the $\mathrm{Hg}$ permeation cell $(200 \mathrm{ug} / \mathrm{m} 3)$. Figure 3.7 shows the concentration of $\mathrm{Hg}$ in the gas leaving the adsorption reactor containing a carbon (A3) with good adsorption capacity. Breakthrough of $20 \mathrm{ug} / \mathrm{m} 3$ of $\mathrm{Hg}$ occurred 19.8 days from the start of the test and the concentration of $\mathrm{Hg}$ in the gas leaving the reactor was zero for 3 days before some $\mathrm{Hg}$ was detected by the $\mathrm{Hg}$ vapor analyzer. However, with a poor $\mathrm{Hg}$ adsorbing carbon (A2) $10 \%$ breakthrough occurred very quickly (2 hours) and the concentration of $\mathrm{Hg}$ in the gas leaving the reactor never went below $7 \mathrm{ug} / \mathrm{m}^{3}$ (Figure 3.8).

The concentration of $\mathrm{Hg}$ in all gasifier carbons tested for $\mathrm{Hg}$ adsorption capacities was determined by XRF. The data agreed with the results from the adsorption testing with poor absorbers with rapid breakthrough capturing little $\mathrm{Hg}$ and good absorbers being loaded with $\mathrm{Hg}$ (Figure 3.9). Carbon $\mathrm{A} 1$ and $\mathrm{A} 3$ contained $1500+\mathrm{ppm} \mathrm{Hg}$ whereas the concentration of $\mathrm{Hg}$ in the rest of the carbons was $200 \mathrm{ppm}$ or less at the $10 \%$ breakthrough point.

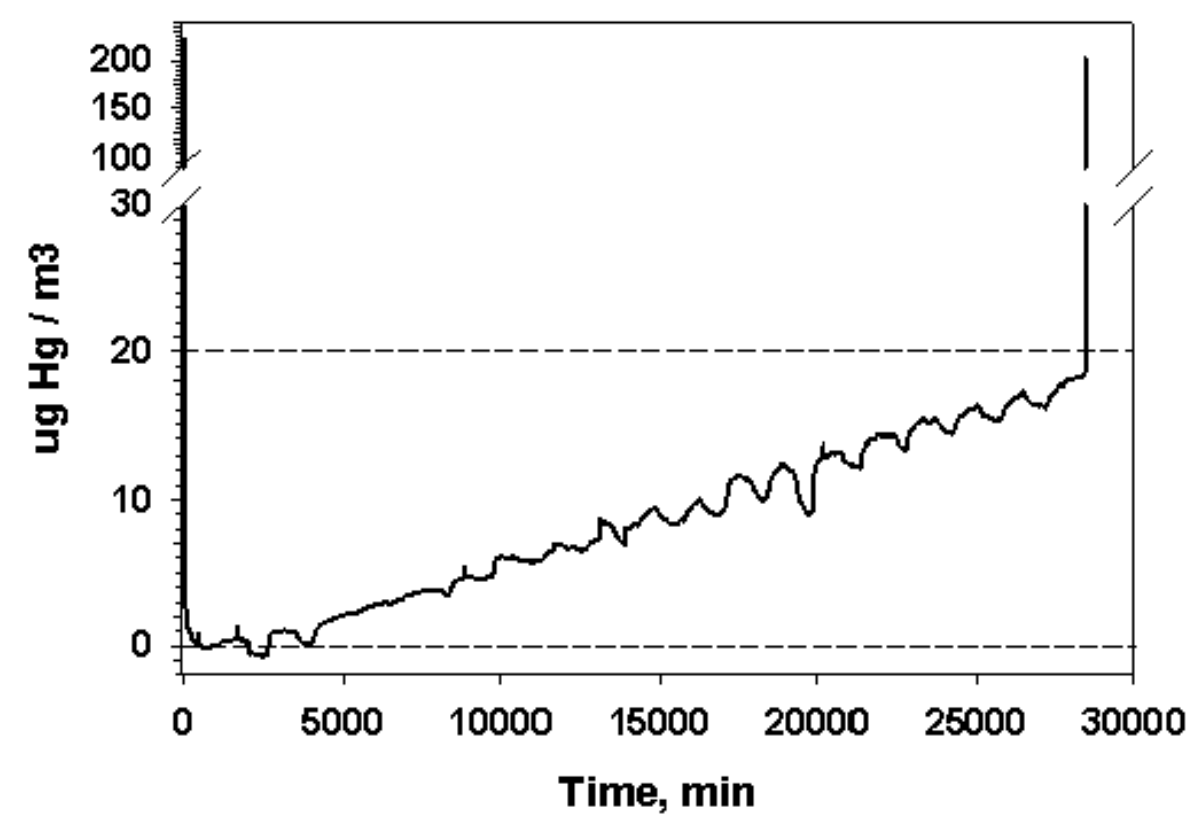

Figure 3.7. Adsorption curve for A3 (-20+80) gasifier carbon. 


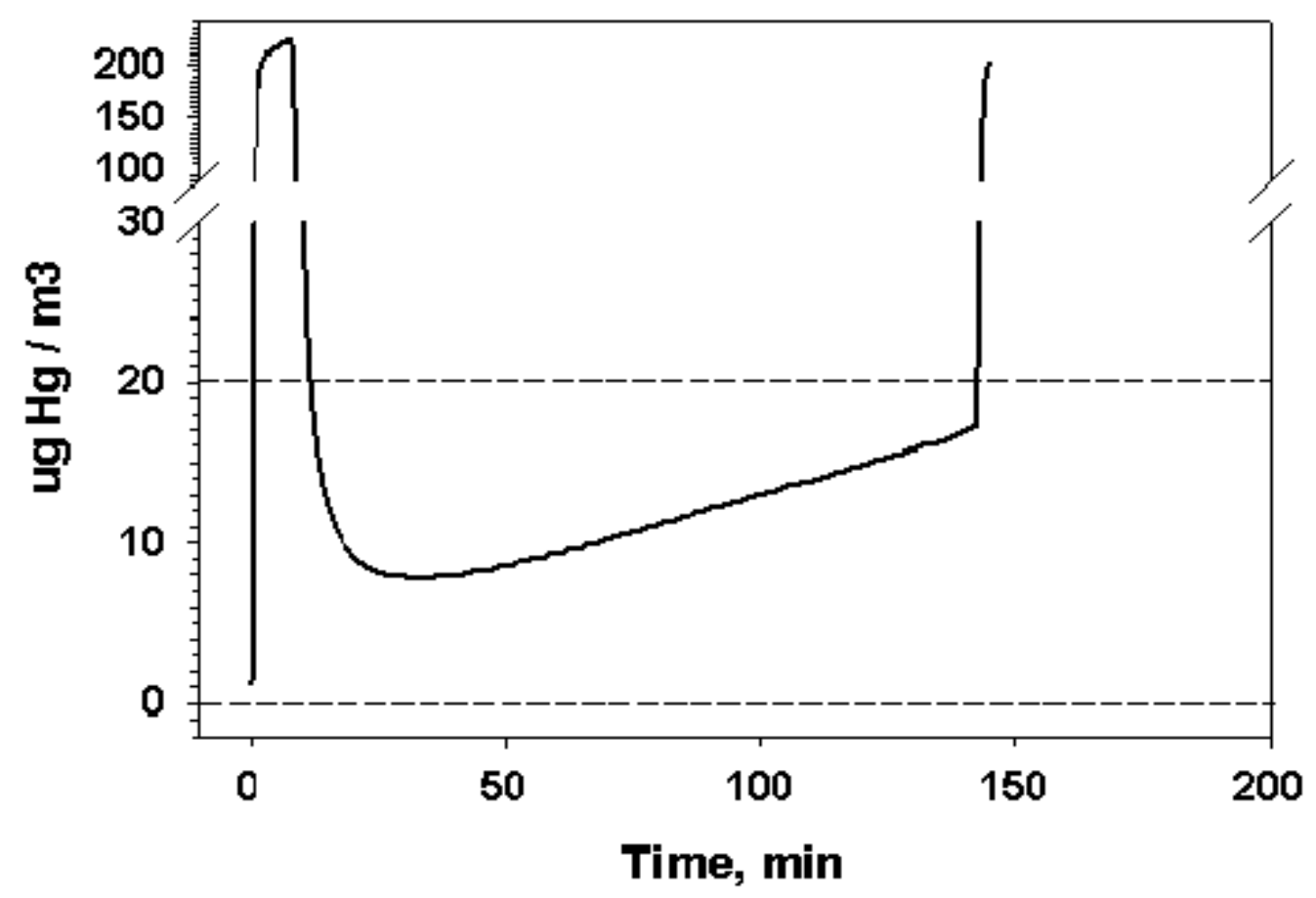

Figure 3.8. Adsorption curve for A2 (-60) gasifier carbon

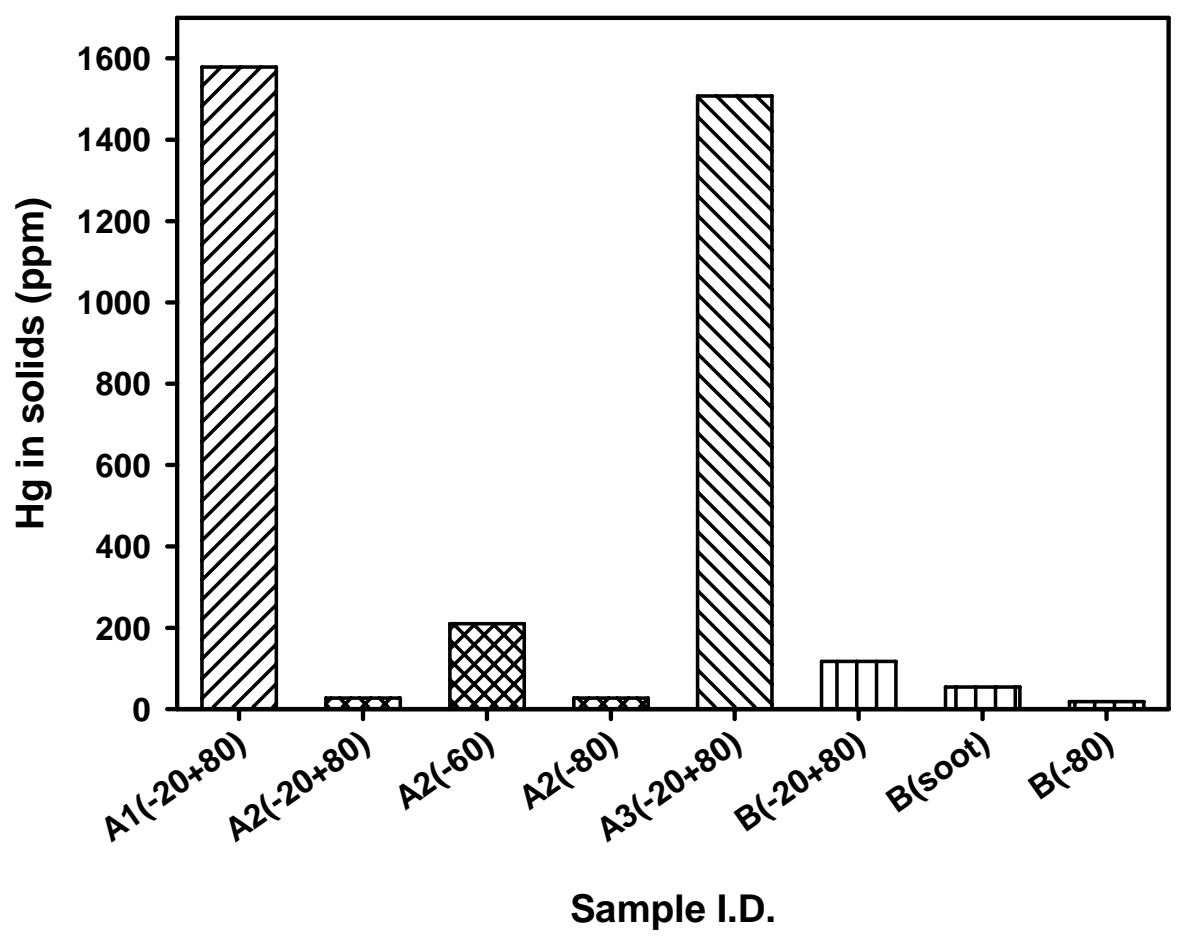

Figure 3.9. Comparison of $\mathrm{Hg}$ capture potential of different gasifier carbons. 


\subsection{Hg adsorption on activated gasifier slag carbons}

3.3.1. Thermal activation. The Energy Institute (EI) at Pennsylvania State University activated thermally and with steam slag samples A1(-20+80), B(-20+80), B(soot), B(-80), and $\mathrm{B}(+20)$ (see EI report). Thermal activation was done at $850^{\circ} \mathrm{C}$ for increasing times $(60,90$, and 120 minutes). Steam activation was done at the same temperature and for 90 minutes. Figure 3.10 shows the $\mathrm{Hg}$ adsorption capacities for all activated slags compared with their untreated parent. The A1 slag which has high $\mathrm{Hg}$ adsorption capability lost $94 \%$ of its $\mathrm{Hg}$ adsorption capacity on activation. This suggests that factors other than higher surface area, increased mesoporosity, and pore volume were important in $\mathrm{Hg}$ adsorption for A1. Mineral content of the slags may play an important role in $\mathrm{Hg}$ adsorption. Except for the B(-80) samples, the other B activated carbons had increased $\mathrm{Hg}$ adsorption at the 60 and 90 min thermal activation times. Steam activation at 90 min improved $\mathrm{Hg}$ adsorption in all B slag carbons except for B $(-20+80)$. There were no correlations between BET SA or micro-, meso-, macro- or total pore volumes and $\mathrm{Hg}$ adsorption capacities.

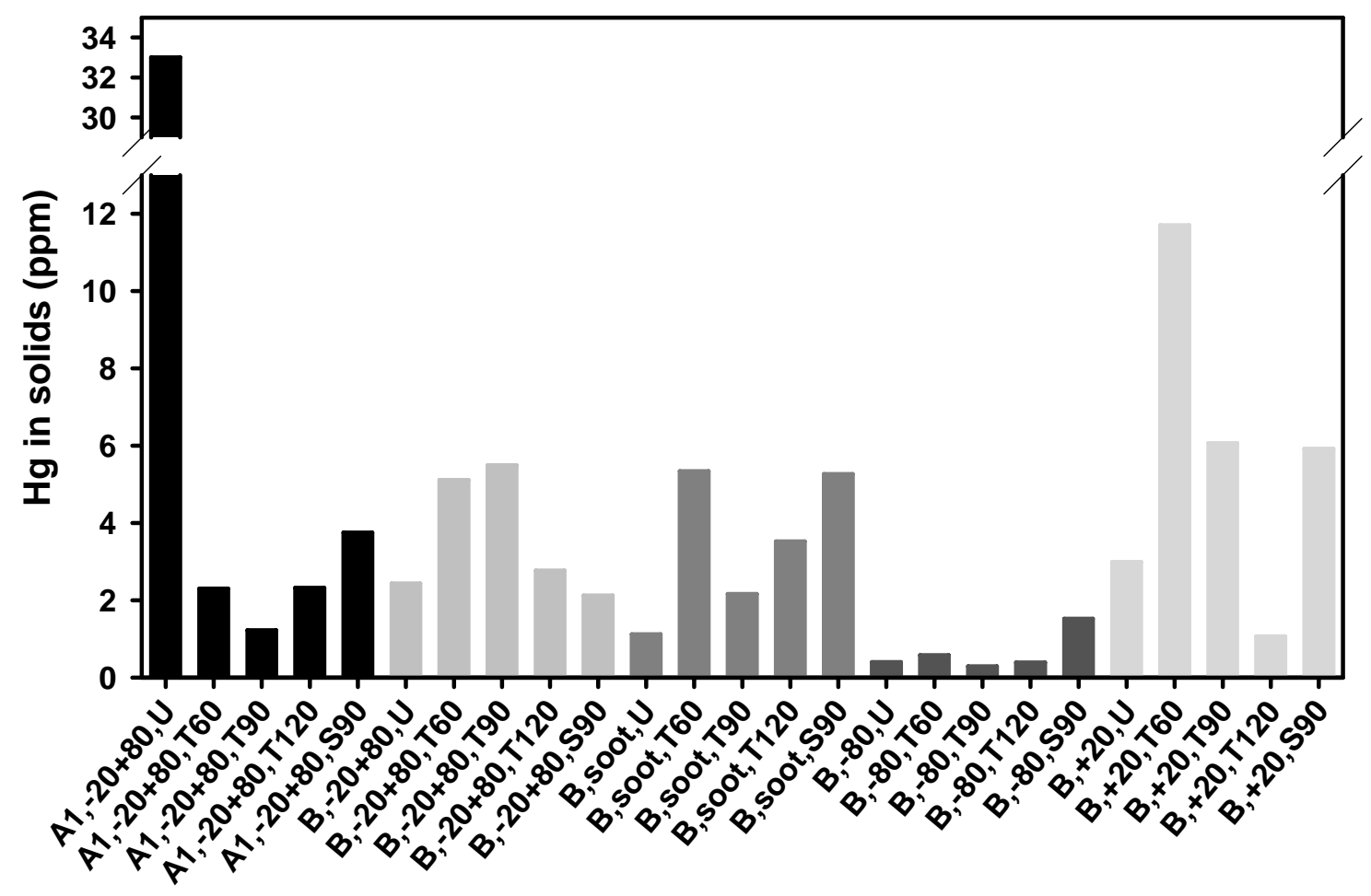

Sample I.D.

Figure 3.10. Hg adsorption capacities of untreated and activated gasifier slag samples.

3.3.2. Chemical activation. Gasifier slag carbons, $\mathrm{A} 1(-20+80)$ and $\mathrm{B}(-20+80)$, were chemically activated at the CAER and tested for Hg adsorption similarly to the EI thermally activated samples. BET SA was decreased for both samples as a result of chemical activation from 109 to 31-80 and 142 to 8-32 for A1 and B respectively. Activation of these samples completely destroyed the $\mathrm{Hg}$ adsorption capability of A1 decreasing it to less than $20 \%$ of the untreated sample. B $(-20+80)$ did not adsorb significant $\mathrm{Hg}$ untreated and continued to have little adsorption capability after chemical activation. 


\subsection{Hg binding sites by XAFS Spectrometry}

3.4.1. Mercury determinations. The size of the $\mathrm{Hg} \mathrm{L}_{\mathrm{III}}$ absorption edge observed for the three samples, $\mathrm{A} 1+\mathrm{Hg}(20+21), \mathrm{A} 2+\mathrm{Hg}(22)$, and $\mathrm{A} 1$, was quite different, and clearly reflected the differences in $\mathrm{Hg}$ concentration of the samples. The control sample exhibited no step whatsoever at 12,284 eV and therefore any mercury in this sample is below the limit of detection of the XAFS experiment (estimated to be significantly less than $1.0 \mathrm{ppm}$ ). The other two samples exhibited a very obvious absorption edge at 12,284 eV. The XANES regions for these two samples, along with that of cubic $\mathrm{HgS}$ (metacinnabar), are shown in Figure 3.11.

The spectra of the two sorbents, A1 and A2, are closely similar and appear most similar to that of $\mathrm{HgS}$ of any of the $\mathrm{Hg}$ compounds in our database (Huggins, et. al., 2003). However, there are significant differences between the spectrum of $\mathrm{HgS}$ and those of the gasifier carbons. There are slight shifts in energy of the absorption edge, the broad peaks above the edge, and the inflection points on the absorption edge. However, when the first-derivative spectra are generated (Figure 3.12) and the inflection point differences, IPD, are compared, the IPD values are found to be virtually the same, $7.6 \pm 0.2 \mathrm{eV}$, for both sorbents and for $\mathrm{HgS}$, indicating that $\mathrm{S}^{2-}$ anions most likely surround the $\mathrm{Hg}^{2+}$ cations in the sorbents.

Both similarities and differences are also seen when the EXAFS/RSF (Radial Structure Function) spectra of the two gasifier slag carbons and that of $\mathrm{HgS}$ are compared. This comparison is shown in Figure 3.13. The peak in the RSF spectrum occurs at about $2.05 \AA$ for cubic $\mathrm{HgS}$, but is displaced by about $0.15 \AA$ to shorter distance for the carbonaceous slags. Such displacements have been noted previously for S-based carbonaceous sorbents (Huggins, et. al., 2003).

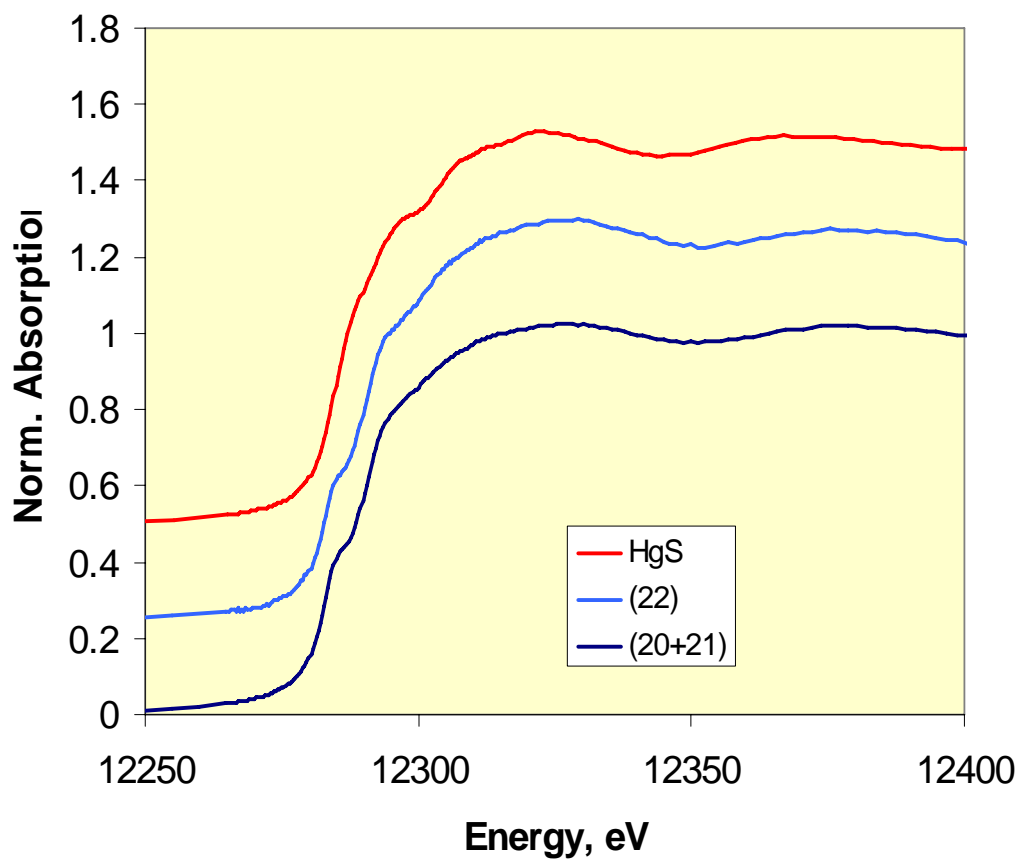

Figure 3.11. Mercury $\mathrm{L}_{\mathrm{III}}$-edge XANES spectra for sorbents and for cubic $\mathrm{HgS}$. 


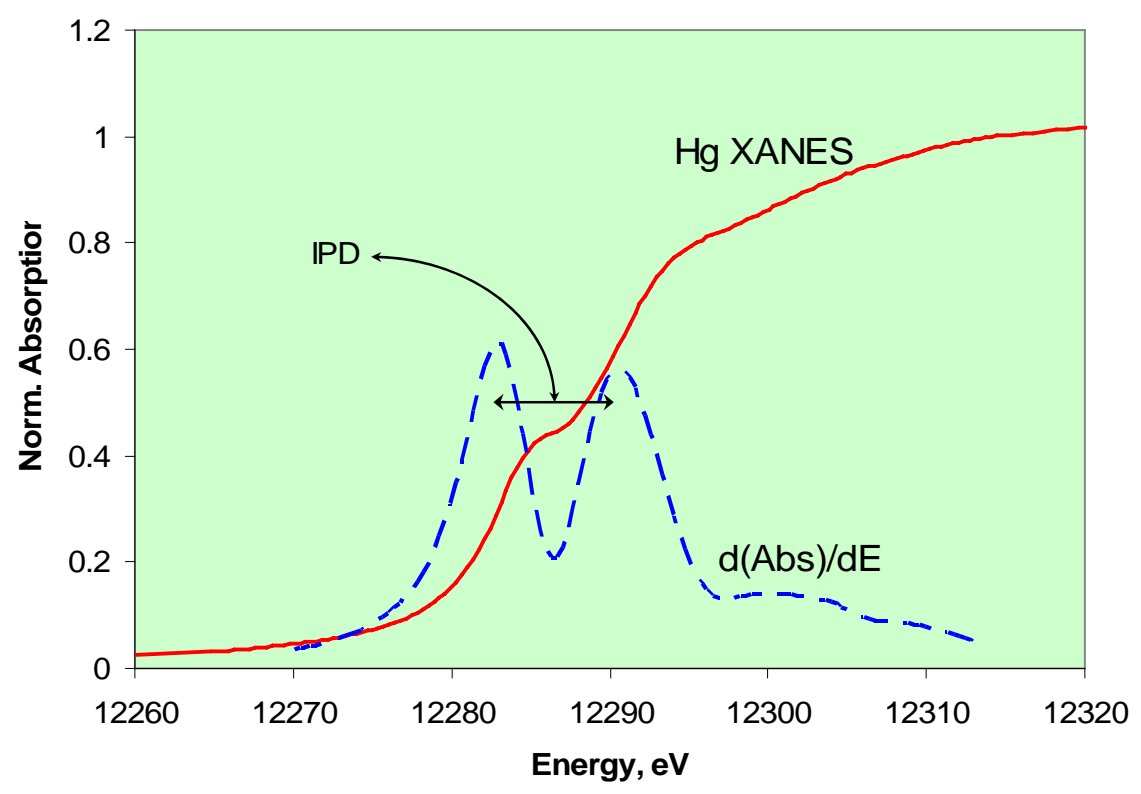

Figure 3.12. Definition and determination of the IPD parameter for Hg XANES spectra. The inflection point difference (IPD) is measured as the separation in energy of the double peak that is obtained by differentiating the Hg XANES spectrum. The data are smoothed before being differentiated, and as discussed elsewhere (Huggins, et. al., 1999), normally the measurement is made using the second derivative.

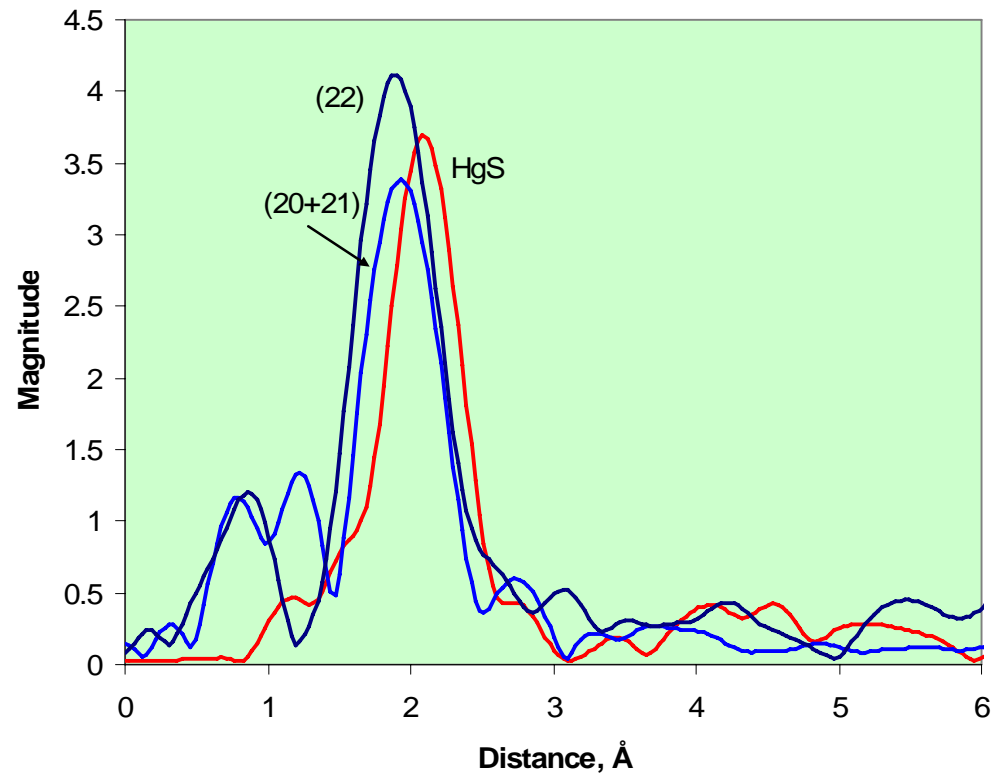

Figure 3.13. EXAFS-RSF spectra for $\mathrm{HgS}$ (red), and the two carbonaceous sorbents, one with a high loading of $\mathrm{Hg}(20+21)$ (light blue), and the other with a much lighter loading of $\mathrm{Hg}(22)$ (dark blue). Note that the peak position of the main peak is displaced for the sorbent samples compared to that for cubic HgS. The other peaks are mostly, if not entirely, artifacts. 


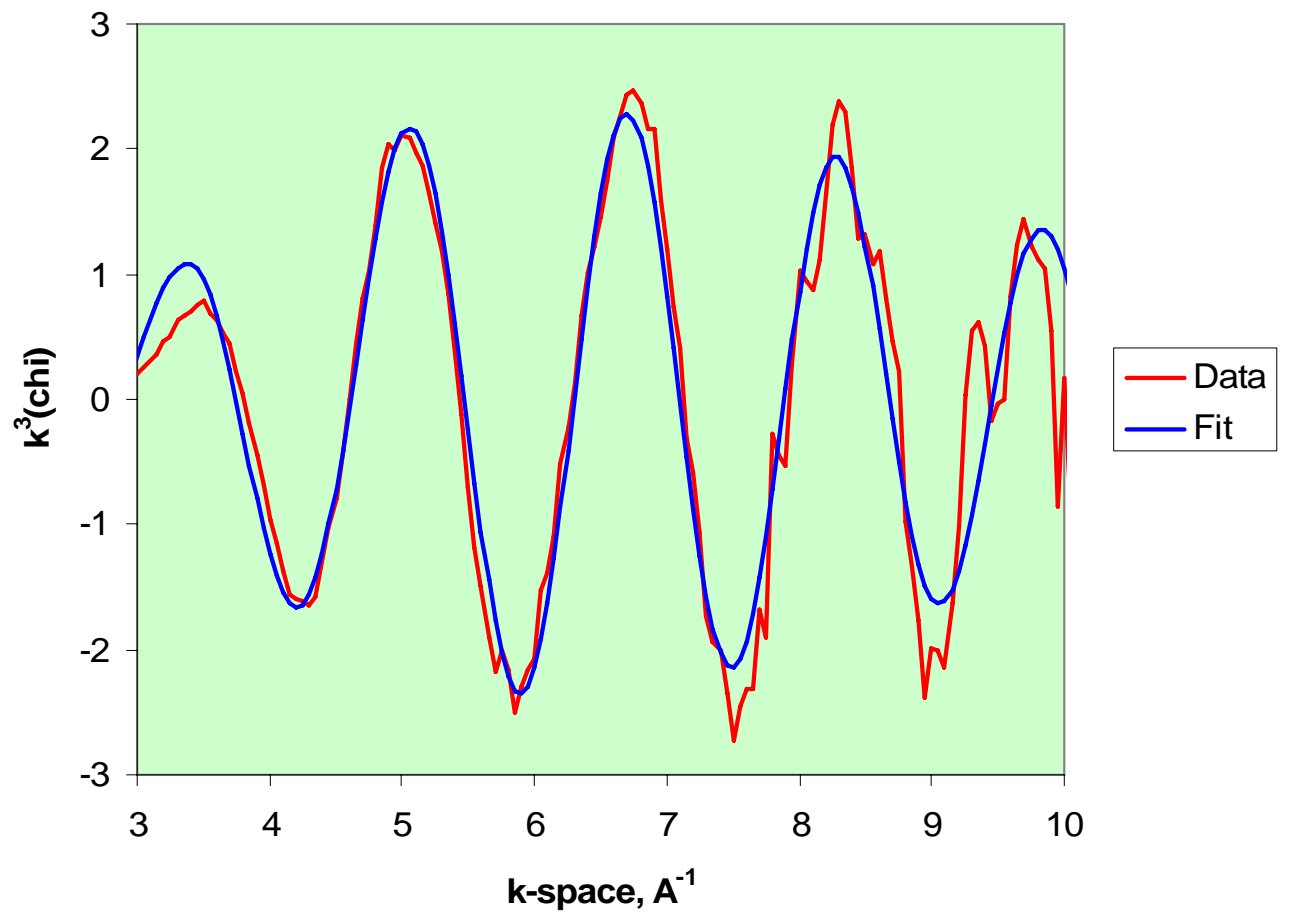

Figure 3.14. Example of least-squares fitting of the $\mathrm{k}^{3}$-weighted chi data region for $\mathrm{Hg}$ in sample $(20+21)$ based on FEFF back-scattering parameters derived from the spectrum of cubic $\mathrm{HgS}$.

Some more advanced fitting of the EXAFS-RSF data has been attempted using leastsquares fitting based on FEFF 6 simulation. Basically, this is a procedure that attempts to calculate the EXAFS region of the spectrum based on established X-ray scattering data for different combinations of elements. In this case, we calculated the FEFF X-ray scattering data for $\mathrm{HgS}$ based on its known crystal structure (space group F -4 3m, $\mathrm{a}_{0}=5.8517 \AA$ ) using the data provided by Wykcoff, 1960. The least-squares fitting was performed using a software package developed by S. Webb of SSRL. Once we had derived various fitting parameters based on the EXAFS data for $\mathrm{HgS}$, we then refined such parameters based on the spectra obtained for the two slag carbons. An example of this kind of fitting is shown in Figure 3.14 and the results are summarized in Table 3.1.

From such fitting, one may derive values for the average Hg-nearest neighbor distance ${ }^{\circledR}$, the approximate coordination number $(\mathrm{N})$, the Debye-Waller factor $\left(\sigma^{2}\right)$ for the $\mathrm{Hg}-\mathrm{X}$ bond, and the energy offset $\left(\mathrm{E}_{0}\right)$. It is clear that, based on the assumption that sulfide anions are the nearest neighbor to the Hg atoms, the distance, coordination number, and Debye-Waller factor are reduced for the $\mathrm{Hg}$ bound to the carbonaceous solid compared to the standard material. The difference of $0.12 \AA$ in bond length is a significant difference and must reflect the difference between the 3-dimensional bonding experienced by $\mathrm{Hg}$ in $\mathrm{HgS}$ and the 2-dimensional surface bonding experienced by $\mathrm{Hg}$ in the sorbents. It is well known that the same M-X bond is often significantly shorter in structures of lower coordination number. Interestingly, based on the fitting results in Table 3.1, the coordination number is clearly reduced for the $\mathrm{A} 1+\mathrm{Hg}$ sample compared to that for the other two samples. 
Table 3.1. XAFS IPD Values and Parameters from least-squares coordination shell fitting

\begin{tabular}{lllllllll}
\hline Sample & $\mathbf{I P D}, \mathbf{e V}$ & $\mathbf{F i t}^{*}$ & $\mathbf{S}_{\mathbf{0}}{ }^{2 * *}$ & $\mathbf{N}^{* *}$ & $\mathbf{E}_{\mathbf{0}}, \mathbf{e V}$ & $\mathbf{R}, \AA$ & $\mathbf{\sigma}^{\mathbf{2}}$ & $\mathbf{R}$ Factor \\
\hline $\mathbf{H g S}$, cubic & 7.6 & $\mathbf{R}$ & 0.23 & 4 & 5.0 & 2.51 & 0.013 & 0.040 \\
& & $\mathbf{k}$ & 0.21 & 4 & 5.4 & 2.51 & 0.012 & 0.095 \\
$\mathbf{( 2 0 + 2 1 )}$ & 7.6 & $\mathbf{R}$ & 0.23 & 2.1 & 3.3 & 2.39 & 0.007 & 0.016 \\
& & $\mathbf{k}$ & 0.21 & 2.2 & 3.6 & 2.39 & 0.007 & 0.079 \\
& & & & & & & & \\
$(\mathbf{2 2})$ & 7.5 & $\mathbf{R}$ & 0.23 & 3.5 & 3.0 & 2.39 & 0.010 & 0.037 \\
& & $\mathbf{k}$ & 0.21 & 3.5 & 3.6 & 2.39 & 0.009 & 0.221 \\
\hline
\end{tabular}

*Least-squares fitting performed on RSF spectrum ${ }^{\circledR}$, over range from $1.4-2.7 \AA$, or on chi spectral data $(\mathrm{k})$, over range from $3-10 \AA^{-1}$. The results are reasonably consistent between the two types of fit.

$* * \mathrm{~S}_{0}{ }^{2}$ assumed variable, $N$ assumed constant for fitting of $\mathrm{HgS}$ spectra; $\mathrm{S}_{0}{ }^{2}$ assumed constant, $\mathrm{N}$ assumed variable for fitting of $\mathrm{Hg}$ sorbents.

The $\mathrm{R}$ factor is a measure of the quality of the fit and is determined by the signal/noise ratio of the data as well as the adequacy of the fit.

3.4.2. Sulfur and Chlorine determinations. Sulfur and chlorine XANES spectra are shown in Figures 3.15 and 3.16 respectively. The sulfur XANES spectra of the two gasifier carbons, $\mathrm{A} 1+\mathrm{Hg}(20+21)$ and $\mathrm{A} 2+\mathrm{Hg}(22)$, are very different; however, the S XANES spectrum of sample $(20+21)$ is closely similar to that of the control sample. It would appear that the control sample contains slightly more elemental sulfur and thiophenic sulfur forms and slightly less sulfate than the sorbent sample $(20+21)$. The sorbent sample (22) is quite different. The sulfur is largely present as one or more metal sulfides, with only very minor amounts of thiophenic and sulfate sulfur forms present and essentially no elemental sulfur.

The three chlorine XANES spectra are different one from another. Again, the control sample and the sorbent sample $(20+21)$ appear to be most similar; however, the sharp peaks on the low-energy side of the main absorption peak occur at different positions $(-4.8 \mathrm{eV}$ and -3.6 $\mathrm{eV}$, respectively) and are of significantly different intensity. The chlorine XANES spectrum of sorbent sample (22) is significantly weaker than those of the other two samples. However, the small sharp peak at low energy for this sample occurs at about the same position as the much larger sharp peak in the spectrum of sample $(20+21)$. The presence of these narrow peaks at this low energy indicates the presence of either organochlorine or hypochlorite $\left(\mathrm{OCl}^{-}\right)$compounds in the sorbent samples (Huggins and Huffman, 1995). Sulfur and not chlorine was the focus of the EXAFS analysis as part of this project so the chlorine work was extra but clearly indicated that the control A1 (26) and the Hg loaded A1 (20+21) had similar but slightly different chlorine composition possibly indicating that chlorine played a role in $\mathrm{Hg}$ adsorption on the A1 sample. Sample A2 (22) had a weaker XANES spectrum consistent with the very low chlorine content of the sample. 


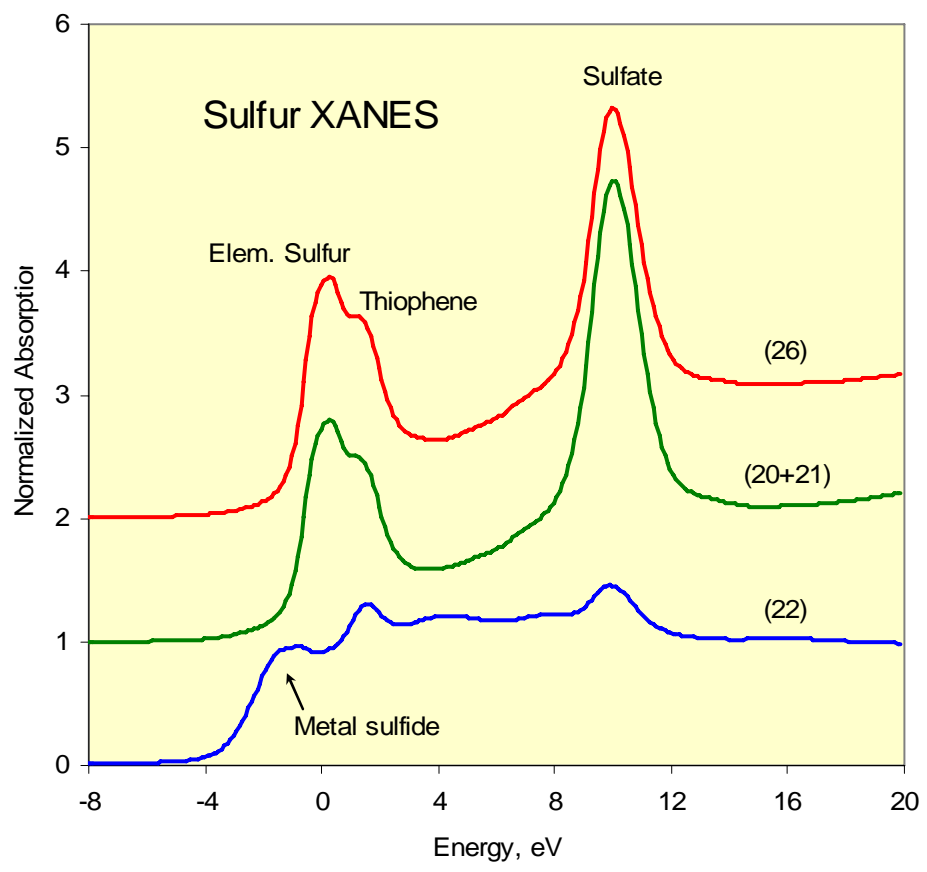

Figure 3.15. Sulfur XANES spectra for the two sorbent samples, $(20+21)$ and (22), and for the control sample, (26). The peaks are identified as arising from elemental sulfur, thiophenic sulfur, sulfate sulfur and metal sulfide. The sulfur forms in (22) largely arise from metal sulfide, which is absent from the other two samples. The zero-point of energy corresponds to $2,472 \mathrm{eV}$.

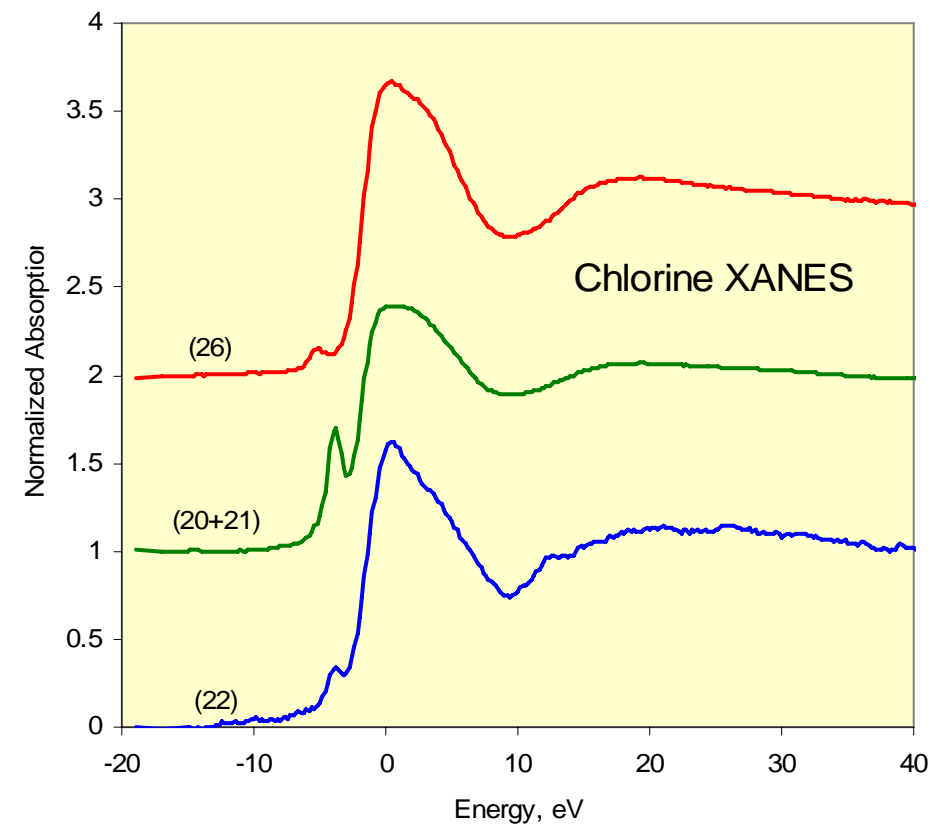

Figure 3.16. Chlorine XANES spectra for the two sorbent samples, $(20+21)$ and (22), and for the control sample, (26). Note the presence of the sharp peak on the low-energy side of the main peak, especially for sample $(20+21)$. The zero-point of energy corresponds to $2,825 \mathrm{eV}$. 


\subsection{NO$\underline{x}$ adsorption capacities for all as received gasifier slags}

All slag samples were submitted to $\mathrm{NO}_{\mathrm{X}}$ adsorption capacity testing using TGMS and were compared to a commercial activated carbon, HGR. The $\mathrm{NO}_{\mathrm{X}}$ adsorption potential of the materials was compared as the weight gained by the sample in response to exposure to an adsorption gas containing NO. HGR weight gain was $6 \%$ which can be attributed nearly completely to physically and chemically bound $\mathrm{NO}_{\mathrm{X}}$ as determined during the desorption phase of the testing. The majority of the $\mathrm{NO}_{\mathrm{X}}$ adsorbed onto the materials was chemically bound with desorption occurring only on heating. $\mathrm{NO}_{\mathrm{X}}$ was released at a maximum rate at $120^{\circ} \mathrm{C}$. Determination of the identity of the desorbed material was by simultaneous MS. The identification of $\mathrm{NO}_{2}$ as the primary desorbed gas was done by determining the mass ratio of amu 30 to 46 as detected by the MS. Amu 30 is the primary mass ion for both $\mathrm{NO}$ and $\mathrm{NO}_{2}$ but amu 46 is present only when $\mathrm{NO}_{2}$ is evolved. Previous work in this area at the CAER has shown that $\mathrm{NO}$ in the presence of $\mathrm{O}_{2}$ and activated carbon is adsorbed as $\mathrm{NO}_{2}$ and $\mathrm{NO}_{2}$ is the primary gas evolved (Rubel, et. al., 1995).

Several of the slag samples, B (-20+80), B (soot), and B (filter cake), adsorbed $40 \%$ of the $\mathrm{NO}_{\mathrm{X}}$ adsorbed by the commercial activated carbon, HGR (Figure 3.17). It was also interesting to note that the adsorption of $\mathrm{Hg}$ on sample $\mathrm{Al}(-20+80)$ increased its $\mathrm{NO}_{\mathrm{X}}$ adsorption capacity by $27 \%$. Figure 3.17 shows the weight gain by the slags which was related to the $\mathrm{NO}_{\mathrm{X}}$ adsorbed. $\mathrm{NO}_{\mathrm{X}}$ adsorption correlated with the BET SA and micropore volumes of the samples (Figures 3.18 and 3.19). This is consistent with work which has shown that $\mathrm{NO}_{\mathrm{X}}$ adsorption on activated carbons is mainly in micropores with the optimum pore size of approximately 11 angstroms (Kaneko, K. et. al, 1988). The Hg-laden A1 (-20+80) sample had an increased in SA, total pore volume and micropore volume over the A1 sample without $\mathrm{Hg}$. BET SA, total pore volume and the micropore volume increased from 109 to $199 \mathrm{~m}^{2} / \mathrm{g}, 0.068$ to $0.081 \mathrm{~cm}^{3} / \mathrm{g}$ and 0.25 to $0.34 \mathrm{~cm}^{3} / \mathrm{g}$ respectively when $\mathrm{Hg}$ was loaded onto the sample. Previous work at the CAER has suggested that partial filling of a meso and macropores with $\mathrm{Hg}$ would produce some smaller spaces (micro-pores or equivalent spaces) for $\mathrm{NO}_{\mathrm{X}}$ adsorption (Rubel et al, 2005). The increase in surface area, pore volume, and micro-pore volume observed for Hg-laden A1 support this conclusion. Additionally published work has strongly suggested that $\mathrm{Hg}$ is adsorbed as $\mathrm{Hg}^{++}$ even from simulated flue gas containing only $\mathrm{Hg}^{0}$ and that it is ionically bound to the surface of the sorbent (Huggins, et. al. 2003). Our work at the CAER agrees with chemical bonding of $\mathrm{Hg}$ on sorbents since it is only released above $300^{\circ} \mathrm{C}$ during heating (Rubel, et. al., 2003). The ionic binding of $\mathrm{Hg}$ to the surface of pores will result in changes in the characteristics of the pores which the data shows makes the pore more suitable for $\mathrm{NO}_{\mathrm{X}}$ adsorption.

3.6. NOx adsorption capacities of activated slag samples

Two slag, A1(-20+80) and B(-20+80), and their activated samples were tested for their $\mathrm{NO}_{\mathrm{X}}$ adsorption capacities (Figure 3.20). It was expected that activation would increase the $\mathrm{NO}_{\mathrm{X}}$ adsorption capacities since $\mathrm{NO}_{\mathrm{X}}$ adsorption has been shown to occur in micropores (Kaneko, et. al., 1988) but this was not the case for the gasifier slag activated samples. The likely cause for this was the increased SA and pore volume of these samples was mostly related to increases in mesopore volume and not micropores (Figures 3.5 and 3.6). No correlations were found between $\mathrm{NO}_{\mathrm{X}}$ adsorption and BET SA, and pore volumes (total, macro, meso, and micro) for the activated samples. Values for $\mathrm{r}^{2}$ were between 0.24 and 0.53 . 


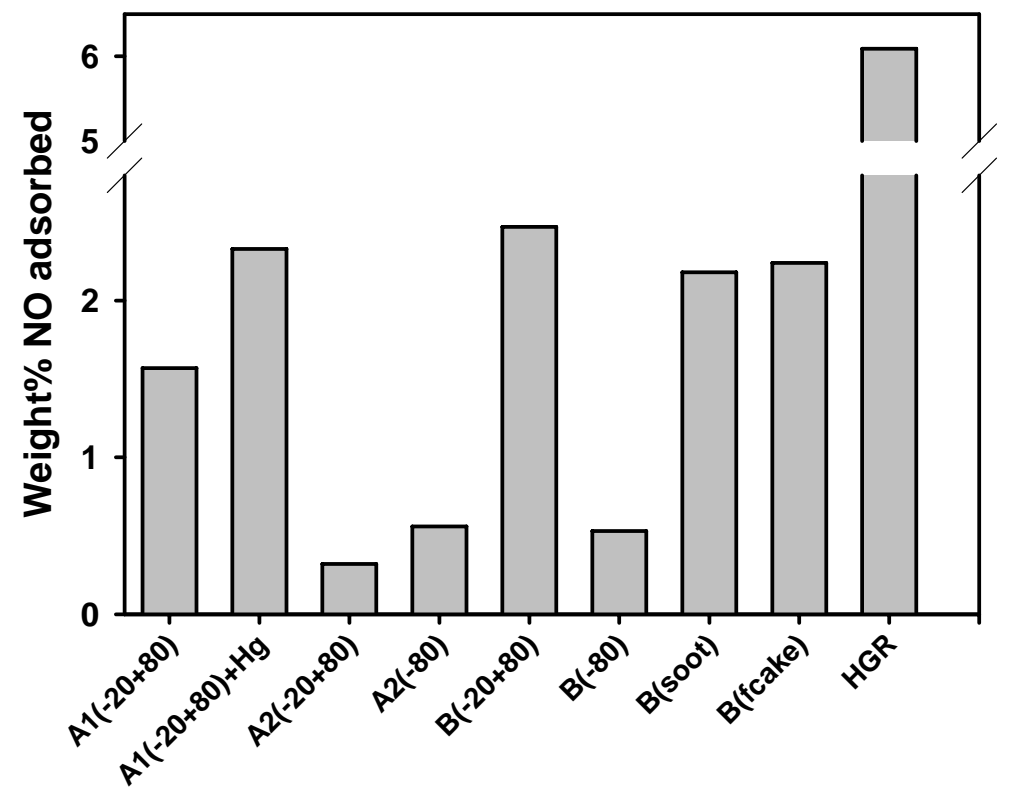

Sample

Figure 3.17. $\mathrm{NO}_{\mathrm{X}}$ adsorption capacities of gasifier slag samples.

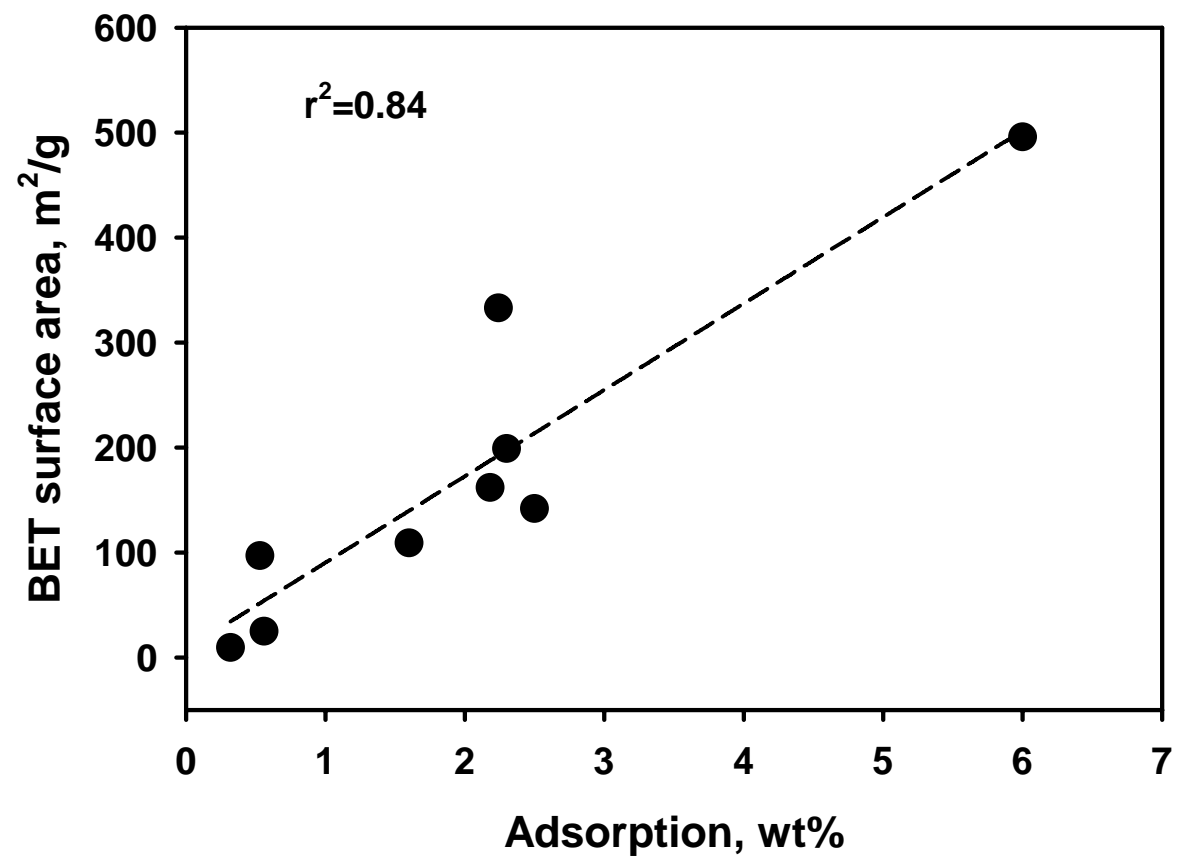

Figure 3.18. Correlation between $\mathrm{NO}_{\mathrm{x}}$ adsorption capacity and BET SA gasifer slags. 


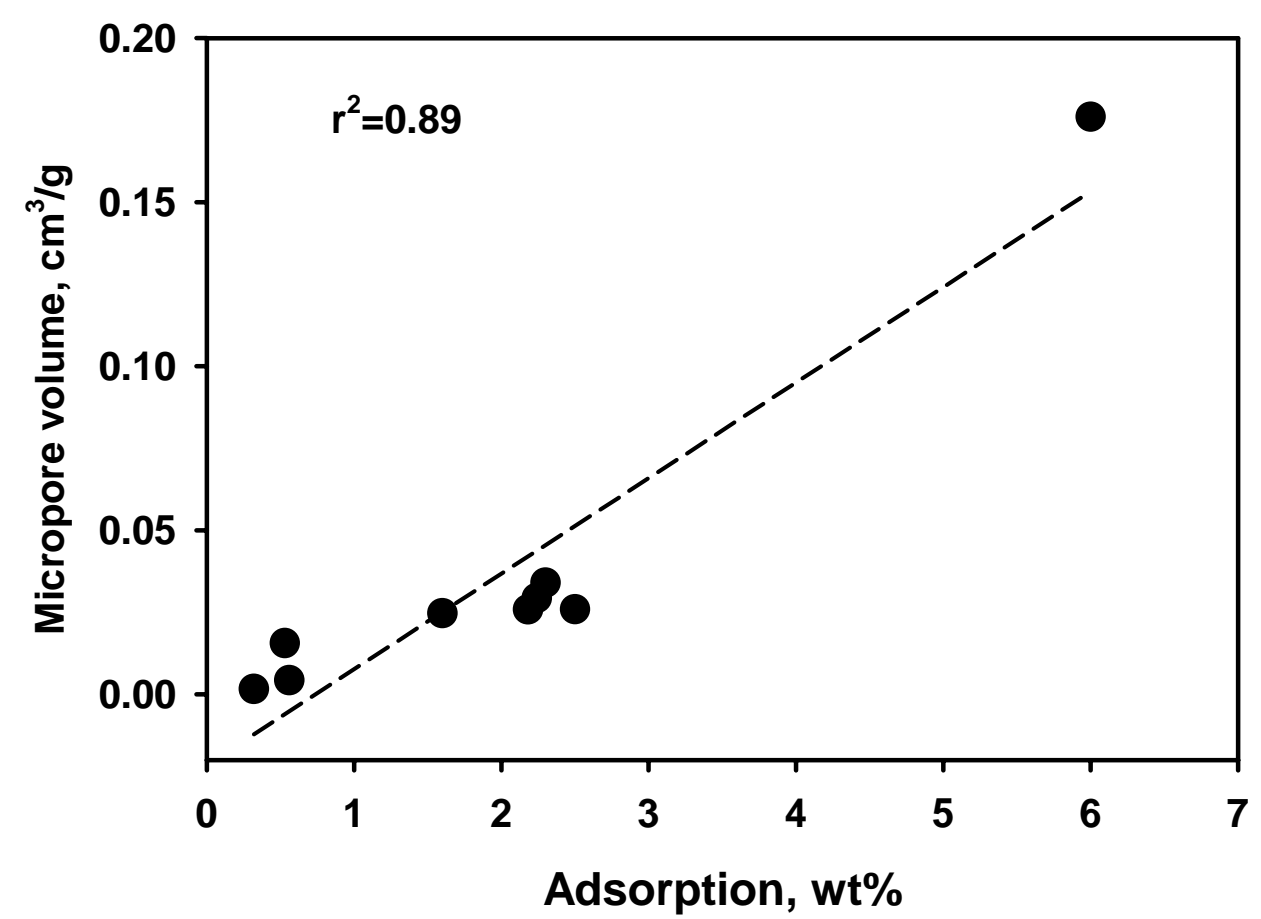

Figure 3.19. Correlation between $\mathrm{NO}_{\mathrm{X}}$ adsorption capacity and micropore volume of gasifier slags.

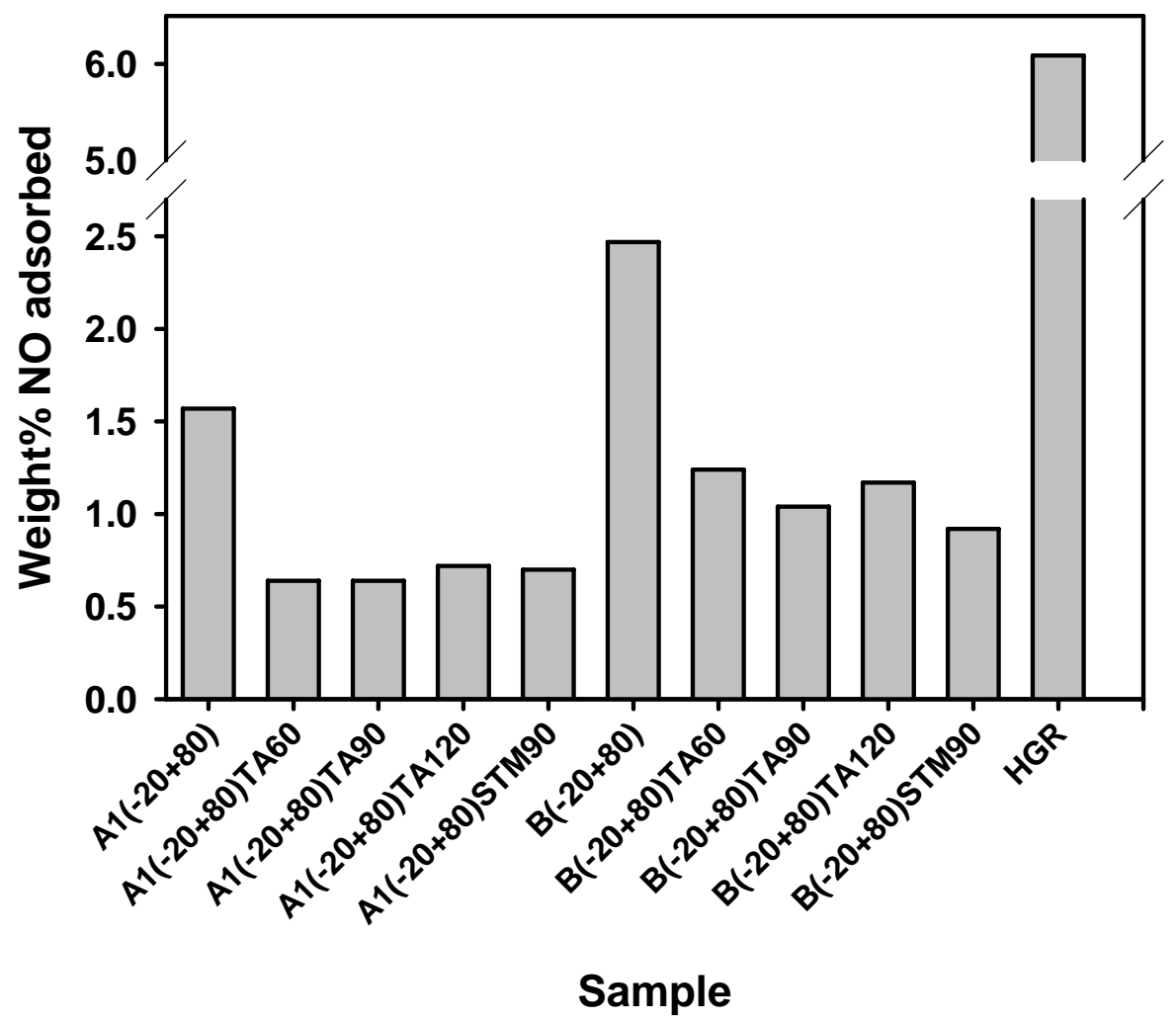

Figure 3.20. $\mathrm{NO}_{\mathrm{X}}$ adsorption on untreated and activated gasifier slag carbons. 


\subsection{Adsorption of $\mathrm{Hg}$ in a simulated flue gas}

As a final test of the use of gasifier slag carbons for $\mathrm{Hg}$ adsorption, three of the slags were tested in a simulated flue gas containing $\mathrm{NO}$ and $\mathrm{SO}_{2}$. Figure 3.21 shows a comparison of the adsorption of $\mathrm{Hg}$ onto the three slag samples (A1, A2, and B) in the simulated flue gas. All samples had the same particle size distribution. They were compared with the commercial activated carbon, HGR, developed for $\mathrm{Hg}$ adsorption. As with $\mathrm{Hg}$ adsorption on these samples in air, the A1 slag out performed the other slag carbons. However, the presence of $\mathrm{NO}$ and $\mathrm{SO}_{2}$ in the adsorption gas decreased its adsorption capacity $40 \%$. Surprisingly the HGR sample did well in air but poorly in the presence of $\mathrm{NO}$ and $\mathrm{SO}_{2}$ under the conditions studied. This sample was tested multiple times with consistent lower results.

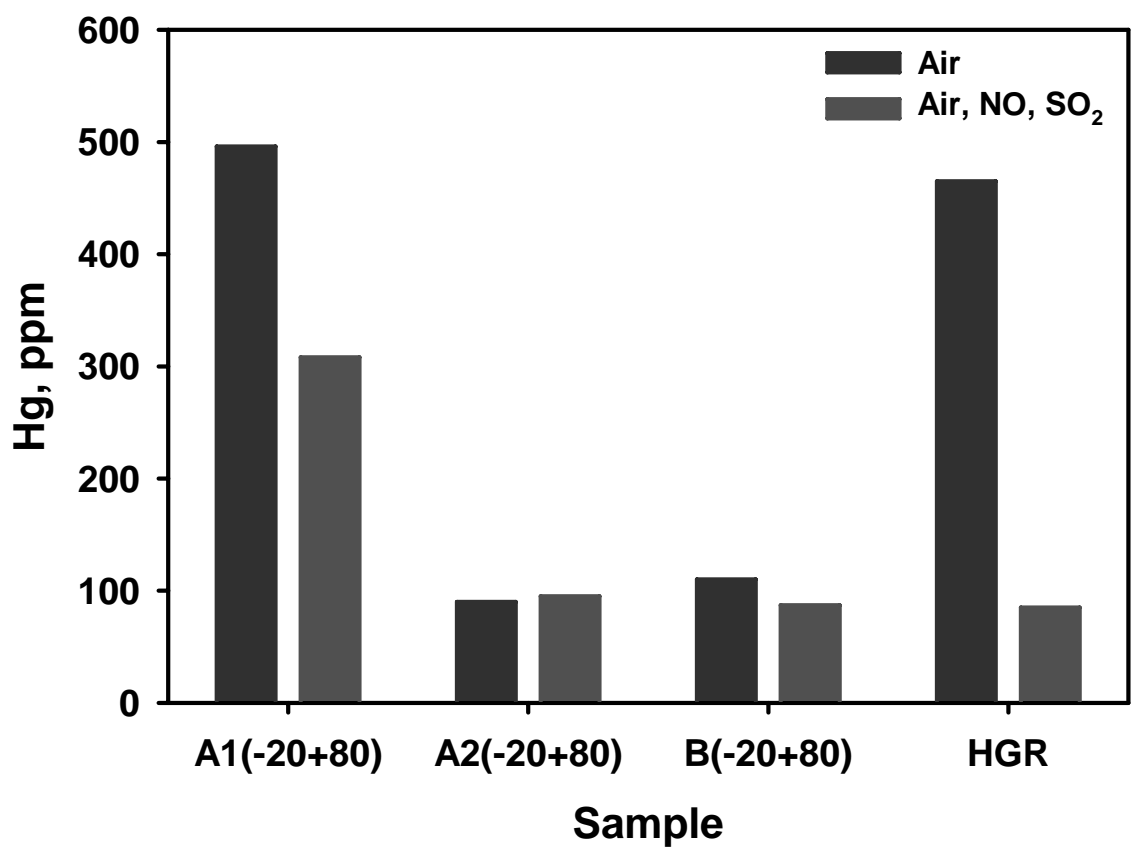

Figure 3.21. Hg adsorption on gasifier slag carbon in simulated flue gas vs. air.

\subsection{Leachability of $\mathrm{Hg}$ from $\mathrm{Hg}$-laden gasifier slag carbons}

A $50 \mathrm{~g}$ sample of A1 (-20+80) was exposed to $200 \mathrm{ug} / \mathrm{m}^{3}$ of $\mathrm{Hg}$ in air for 1 month providing a sample containing $13 \mathrm{ppm} \mathrm{Hg}$. This sample plus a virgin sample of the same slag was leached in distilled \& deionized water in the following amounts: baseline A1: $50.0 \mathrm{~g}$ slag in $228.9 \mathrm{~g}$ of distilled deionized water, liquid/solid $=4.58 ; \mathrm{Hg}$ Loaded A1: $50.0 \mathrm{~g}$ slag in $218.0 \mathrm{~g}$ of distilled deionized water, liquid/solid $=4.36$. The samples were leached for 48 hours and filtered at 0.45 microns. The $\mathrm{Hg}$ concentrations in the leachates were: distilled deionized water blank: $<$ $10 \mathrm{ng} / \mathrm{L}$ (parts per trillion); baseline A1: < $10 \mathrm{ng} / \mathrm{L}$ (ppt); Hg loaded A1: $47 \mathrm{ng} / \mathrm{L}$ and $39 \mathrm{ng} / \mathrm{L}$ in duplicate digestions. Converting to original slag: baseline slag: $<40 \mathrm{ng} \mathrm{Hg}$ released $/ \mathrm{kg}$ slag and $\mathrm{Hg}$ loaded slag: $200 \mathrm{ng} \mathrm{Hg}$ released/kg slag. At the conditions studied less than less than $4 \mathrm{e}-4 \%$ of the $\mathrm{Hg}$ captured by the slag was released.

The batch leaching test in this study would only simulate the mercury released from rainwater infiltrating through a pile of the slag. Interpolations to one month or longer would require column leaching studies. The results of this batch leaching test are consistent with other results. Mercury does not readily leach from carbon-rich materials. 


\subsection{Evaluation of Slag as a Supplementary Cementitious Material}

Pozzolanic reactivity of fly ash is assessed using ASTM C 311 . Mortar is prepared using Portland cement, $20 \%$ of which is replaced with fly ash. The material is cured wet for several months and tested for compressive strength. The strength data are then compared with a Portland cement-only mortar (control) and reported as a Strength Activity Index (SAI). The minimum SAI for a fly ash is specified in ASTM C 618. Because ground gasification slags are similar in composition to a Class $\mathrm{F}$ fly ash, the $\mathrm{C} 311$ protocols would be used to assess their reactivity. These tests were performed under another work activity, "Environmentally Safe, Large Volume Utilization Applications for Coal Gasification By-Products" (DE-FC2604NT42203), and specific results are reported under that project.

The +20 mesh (i.e. low-carbon) fractions of the A1 and B slag were given a cursory evaluation for its potential as a pozzolanic concrete admixture. The most important properties to consider are chemical composition, crystallinity, and particle size. In order to be used as a pozzolan the slag would need to be ground to a size similar to Portland cement, that is, with a median particle size of less than 15 microns. The chemical composition determined by XRD analysis of the +20 mesh fraction for each slag is shown below in Table 3.2. According to ASTM C 618, a Class F pozzolan should have a $\mathrm{SiO}_{2}+\mathrm{Al}_{2} \mathrm{O}_{3}+\mathrm{Fe}_{2} \mathrm{O}_{3}$ content of at least $70 \%$, which both of the slags surpass. However, the very high iron content of the B slag will likely decrease its pozzolanic reactivity relative to the A1 slag.

Table 3.2. Chemical Composition of the +20 Mesh Slag Fractions

\begin{tabular}{|l|c|c|}
\hline \multicolumn{1}{|c|}{ Component } & $\mathbf{A 1}$ & $\mathbf{B}$ \\
\hline $\mathbf{S i O}_{\mathbf{2}} \mathbf{( \% )}$ & 53.81 & 38.77 \\
\hline $\mathbf{A l}_{\mathbf{2}} \mathbf{O}_{\mathbf{3}} \mathbf{( \% )}$ & 19.52 & 20.54 \\
\hline $\mathbf{F e}_{\mathbf{2}} \mathbf{O}_{\mathbf{3}} \mathbf{( \% )}$ & 10.89 & 28.18 \\
\hline $\mathbf{C a O}(\mathbf{\%})$ & 3.73 & 2.06 \\
\hline $\mathbf{M g O} \mathbf{( \% )}$ & 1.06 & 0.97 \\
\hline $\mathbf{N a}_{\mathbf{2}} \mathbf{O}(\mathbf{\%})$ & 2.39 & 0.32 \\
\hline $\mathbf{K}_{\mathbf{2}} \mathbf{O}(\mathbf{\%})$ & 2.34 & 2.60 \\
\hline $\mathbf{P}_{\mathbf{2}} \mathbf{O}_{\mathbf{5}}(\mathbf{\%})$ & 0.66 & 0.10 \\
\hline $\mathbf{T i O}_{\mathbf{2}} \mathbf{( \% )}$ & 0.99 & 1.35 \\
\hline $\mathbf{S O}_{\mathbf{3}} \mathbf{( \% )}$ & 0.59 & 1.50 \\
\hline
\end{tabular}

In a commercial pozzolan such as Class $\mathrm{F}$ coal fly ash, the reactivity is highly dependent on the degree of crystallinity: in general, the more crystalline material (i.e. quartz) that is present, the lower the reactivity. Conversely, a higher content of amorphous glassy material generally causes a higher reactivity. Therefore, $\mathrm{x}$-ray diffraction (XRD) analysis was conducted on the +20 mesh slag fractions to qualitatively assess the glass content. The XRD spectrum for the $\mathrm{B}$ slag is shown in Figure 3.22, and is also representative of the A1 slag. The lack of any significant crystalline diffraction lines indicates that the material is very high in glass content and thus should impart a high degree of pozzolanic reactivity (after the material is ground). 


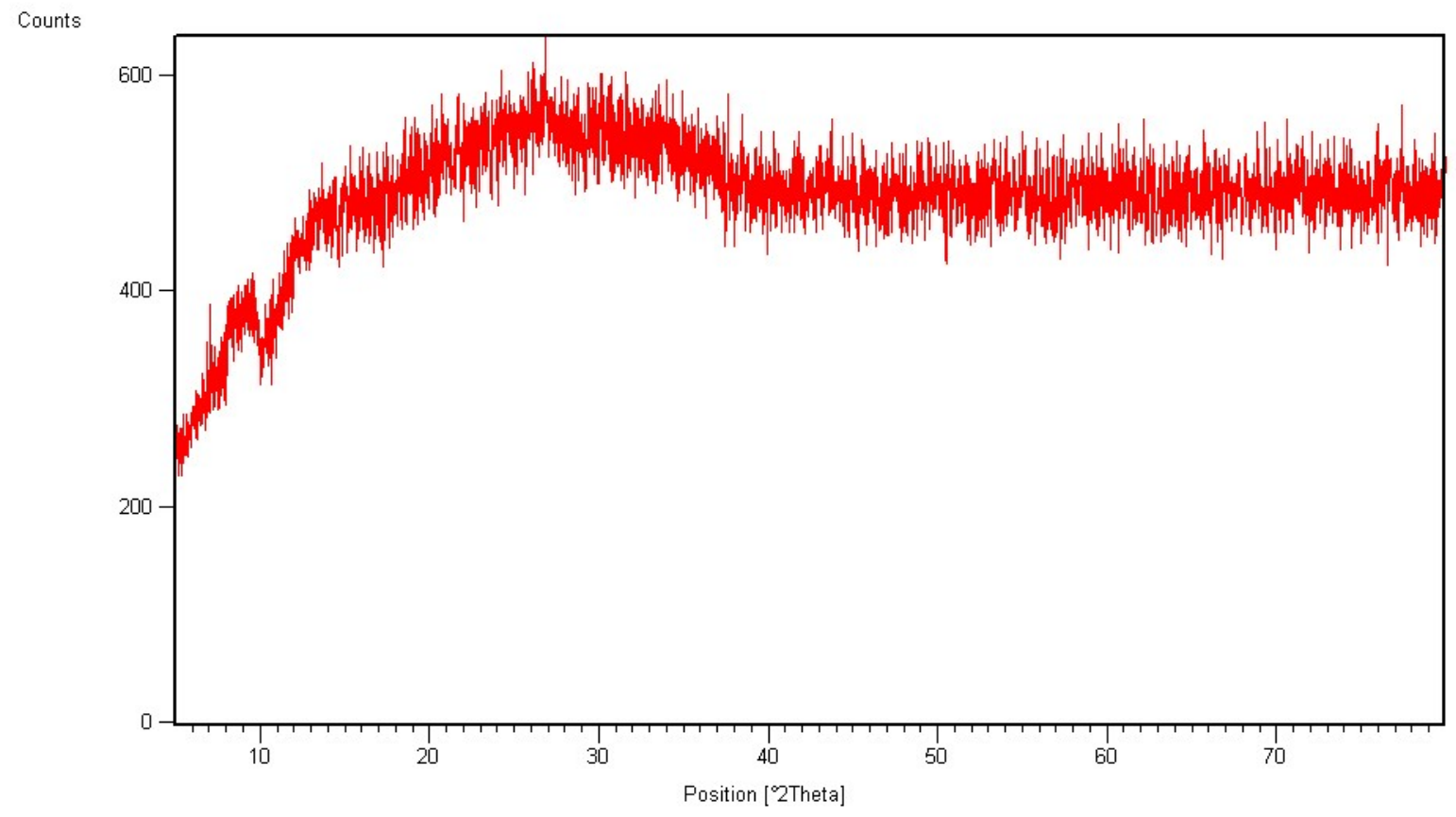

Figure 3.22. XRD spectrum of B slag.

The slags could be used in concrete in two forms: as a Supplementary Cementitious Material (SCM; described above) in a ground form, and as a coarse aggregate component. Testing of the slag as an SCM in concrete would be accomplished using similar procedures as for mortar described above. As a coarse aggregate, the gradation of the slag would be determined and blended with other aggregates to optimize workability. Testing would then be performed to determine if the slag has sufficient mechanical strength in the concrete. Another issue that would need to be addressed is the potential for the slag to cause staining of the concrete due to the presence of iron sulfide in the slag. A test slab would be prepared, cured outdoors and monitored for staining.

3.10. Use of gasifier slag carbon as fillers

The goal of using fillers in polymer matrices is two fold. First, the filler is added with the intent of altering the mechanical and material properties of the polymer. Second, a polymer that contains filler, which is typically a low cost material, is more cost effective than a polymer without filler. Fillers are usually inexpensive materials that are readily available in large quantities (talc, ash, carbon black, etc) depending on the desired enhancement. Fillers intended as material reduction additives (low cost bulk fillers such as talc, calcium carbonate or clays) are typically added in loadings as high as $40 \%$, while fillers targeting enhancements in electrical or thermal properties (carbon blacks) may be added in loadings approaching $20 \%$. Alternatively, fillers that are added to polymers for the purpose of improving mechanical or material properties, such as glass or carbon fibers, are generally added in lesser amounts due to their greater expense. These composite materials are useful for a range of applications; for example, composite materials that are electrically conductive are used as carrier tapes for the electronics industry. Similarly, carbon fiber reinforced polymers are frequently used in sporting goods and other high performance applications. Altering the thermal conductivity of a polymer enables its use as a heat resistant material in consumer electronics housings. 
In the work reported here, molding grade polypropylene and thermoplastic elastomer (TPE-A) were used as the host matrix. These two materials were chosen as representative of polymer systems which are typically used in filled form- polyolefins and elastomers, respectively. During the test for surface resistivity each material tested was insulating except for the TPE-A containing $40 \mathrm{wt} \%$ slag, which achieved conductivity suitable for static dissipation applications (Figure 3.23).

Adding Slag A3 to polypropylene decreased the peak tensile stress as the concentration of the slag increased. (Figure 3.24a) However, the virgin polypropylene (0 wt $\%)$ yielded at a much lower stress (striped bar in Fig. 3.24a); whereas, the composite materials containing Slag A3 yielded very little, if at all. Alternatively, the elastic modulus of the polypropylene steadily increased with increasing concentration of Slag A3. (Figure 3.24b) These results suggested that the Slag A3 has very little reinforcing effect on polypropylene.

\section{Surface Resis tivity of Char A3 Composite Films}

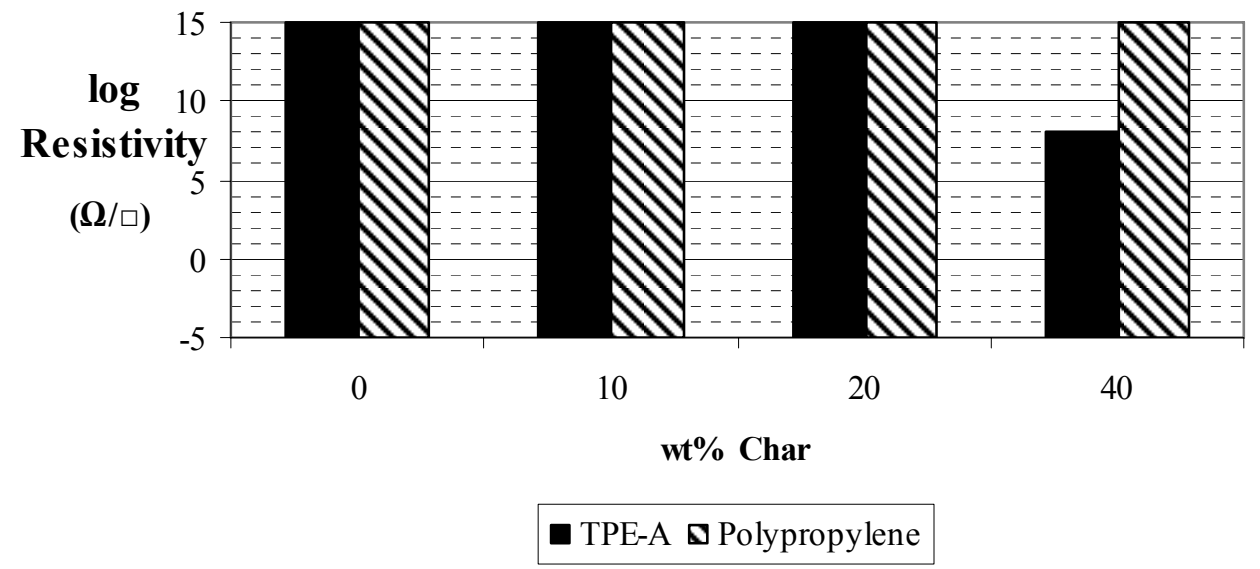

Figure 3.23. Each material was insulating except for the $40 \mathrm{wt} \% \mathrm{~A} 3 \mathrm{in} \mathrm{TPE}-\mathrm{A}$.

Tensile Strength

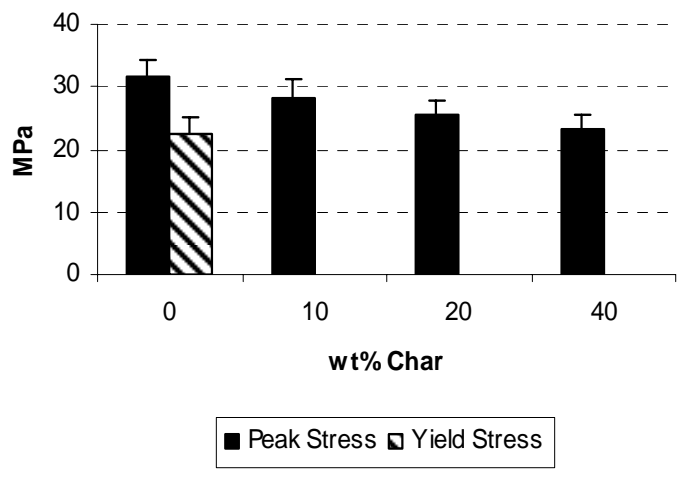

(a)
Elastic Modulus

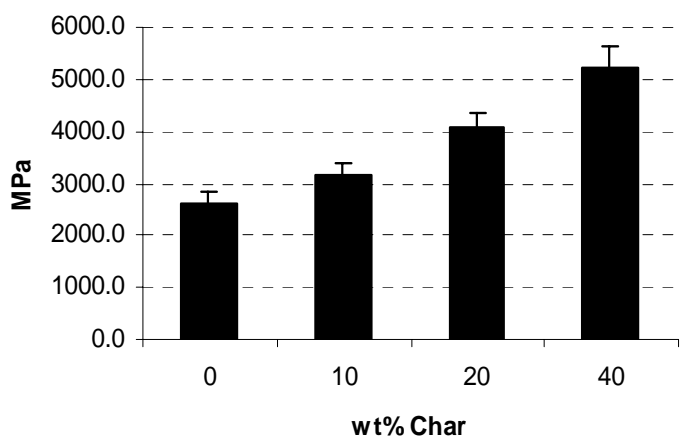

(b)

Figure 3.24. The strength and modulus for polypropylene with Slag A3 were measured with a simple tensile test. 
Similar results were witnessed for TPE-A reinforced with Slag A3. The peak stress decreased sharply with the addition of $10 \mathrm{wt} \%$ Slag A3 (Figure 3.25a) while there was little difference in the peak stresses of TPE-A containing 10, 20, or $40 \mathrm{wt} \%$ Slag A3. Much like polypropylene, the control material ( $0 \mathrm{wt} \%$ TPE-A) yielded below the peak stress; whereas, the materials containing Slag A3 yielded very little. The elastic modulus increased with increasing concentration of Slag A3 additive. (Figure 3.25b) The increasing elastic modulus coupled with decreasing peak stress suggested that the Slag A3 did little to reinforce TPE-A.

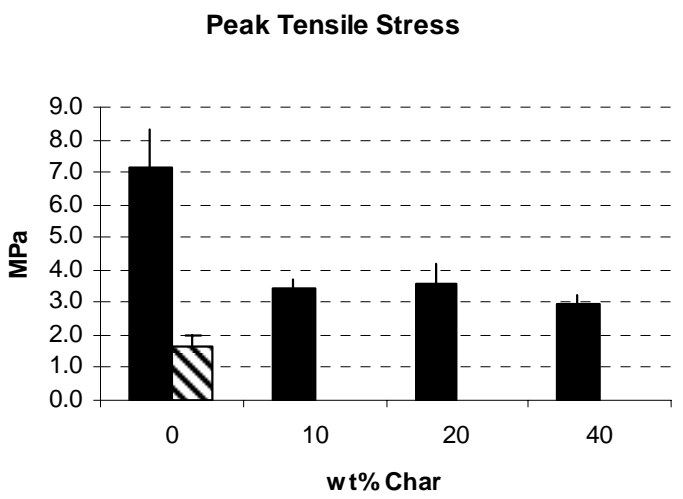

(a)

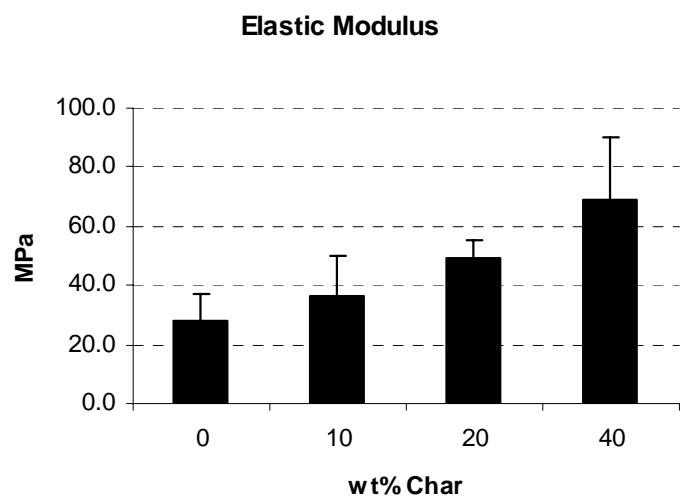

(b)

Figure 3.25. The measured strength and modulus values for TPE-A containing Slag A3 revealed that Slag A3 did little to reinforce the TPE-A matrix.

The addition of Slag A3 to polypropylene slightly increased the thermal diffusivity; furthermore, this increase was dependent on Slag A3 concentration (i.e. the highest diffusivity resulted from the most concentrated sample) (Figure 3.26). The diffusivity decreased as the sample temperature increased, which is normal for polymer systems. For TPE-A, the thermal diffusivity was also altered with the addition of Slag A3 (Figure 3.27). The sample with the highest concentration of Slag A3 (40 wt\%) exhibited the highest level of thermal diffusivity, nearly double all others. This sample was also the most affected by the change in sample temperature (diffusivity markedly decreased with increasing sample temperature). The thermal diffusivity of the less concentrated samples (10 wt $\%$ and $20 \mathrm{wt} \%)$ and the control $(0 \mathrm{wt} \%)$ decreased only slightly at higher sample temperatures.

Comparing these results with those of other composite materials shows that the addition of Slag A3 does not achieve the same mechanical reinforcement as typically seen for glass or carbon fibers. However, it does behave similarly to carbon black or carbonate fillers. The addition of carbon fibers (as well as multiwall carbon nanotubes) to TPE-A increased the tensile strength of the polymer; whereas, the addition of Slag A3 to TPE-A decreased the tensile strength of the polymer. This is not surprising due to the roughly spherical shape and low aspect ratio of the char filler, offering no real expectation for mechanical strength enhancement. Alternatively, the change in electrical conductivity for TPE-A with $40 \mathrm{wt} \%$ Slag A3 was comparable to adding $5 \mathrm{wt} \%$ MWNT (a more expensive filler material) to TPE-A and slightly better than was achieved for carbon fibers and carbon black. However, even at loadings of 40 $\mathrm{wt} \%$, no enhancement of electrical conductivity was observed for the addition of A3 to polypropylene, likely a result of poor dispersion and particle agglomeration. To overcome this effect in polypropylene would require surface modification of the char or use of a wetting agent, 
not atypical of fillers used in unsubstituted polyolefin systems. The effect of Slag A3 on the thermal conductivity of both polymers is comparable to the enhancements seen with the addition of typical graphitic carbon fillers in polymer systems, but at nearly twice a typical loading. While the electrical and thermal conductivity enhancements are desirable, they resulted in undesirable changes in polymer color (darkening to a muddy gray color) and a rougher texture.

\section{Thermal Diffusivity of Char A3 in Polypropylene}

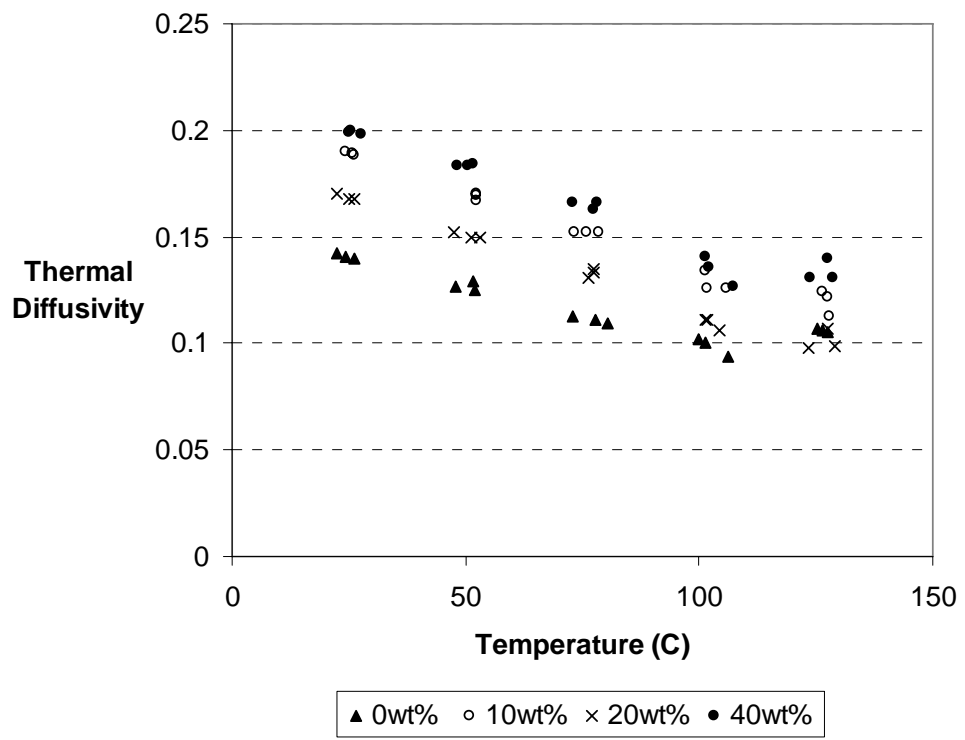

Figure 3.26. The thermal diffusivity increased with the addition of Slag A3 but decreased for all materials with increasing sample temperature.

Thermal Diffusivity of Char A3 in TPE-A

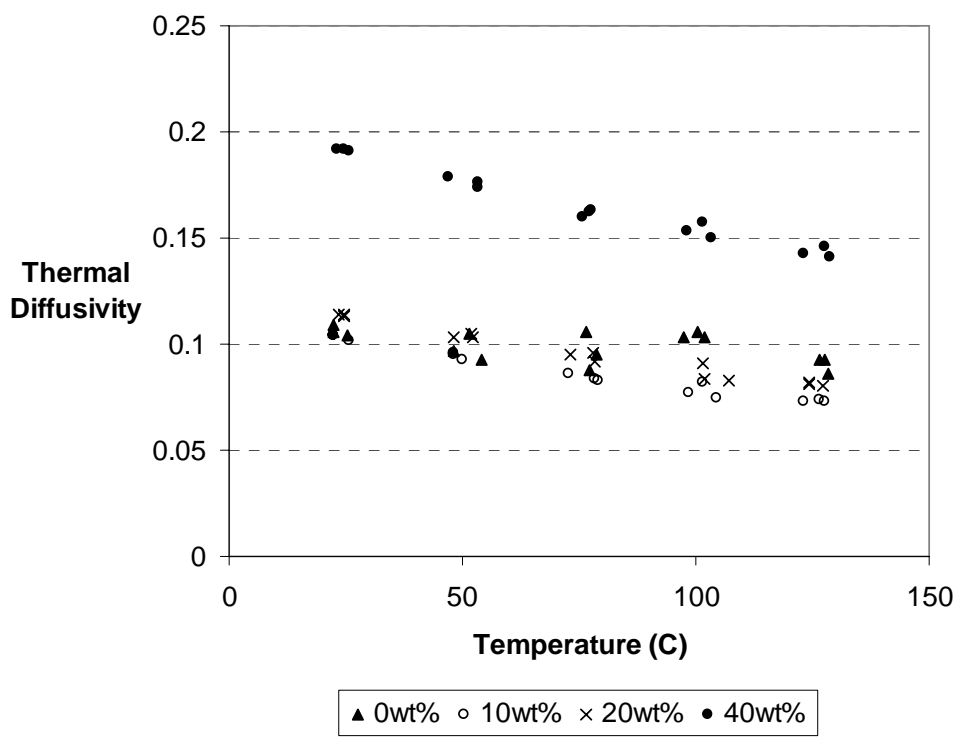

Figure 3.27. The thermal diffusivity of TPE-A remained relatively unchanged with the addition of Slag A3. The exception was the 40wt\% Slag A3 sample, which showed dramatic increases in thermal diffusivity. 


\section{Conclusions}

Two gasifier slag carbons, A1 and A3, had exceptional Hg capture capacities. The data indicated that good $\mathrm{Hg}$ adsorption capacity for these carbons is complex. Some of the chemical characteristics of these gasifer slag carbons which our work and others have suggested as important in $\mathrm{Hg}$ adsorption on carbons are the carbon and sulfur content, the high oxygen content, the presence of a halogen or $\mathrm{SO}_{4}{ }^{-2}$ or both. The carbon contents of A1 and A3 were 46 and $77 \%$ respectively and yet had nearly the same $\mathrm{Hg}$ adsorption capacity. This suggests that carbon content is not the sole determinant of $\mathrm{Hg}$ capture potential. $\mathrm{High} \mathrm{Cl}^{-}, \mathrm{F}^{-}, \mathrm{Br}^{-}$, and oxygen in these sample may be important in $\mathrm{Hg}$ capture since they have been implicated in the oxidation of $\mathrm{Hg}^{0}$ to $\mathrm{Hg}^{+2}$ which is easier to remove from gas streams. $\mathrm{PO}^{-2}$ was also high in both good $\mathrm{Hg}$ sorbents since it has not been implicated in $\mathrm{Hg}$ adsorption previously in the area of $\mathrm{Hg}$ adsorption, more work needs to be done to prove its relativity to the capture of $\mathrm{Hg}$. Impregnation of a sorbent with $\mathrm{PO}^{-2}$ and subsequent $\mathrm{Hg}$ adsorption testing would prove useful in determining $\mathrm{PO}^{-2}$ significance in $\mathrm{Hg}$ capture by carboneous materials.

The surface areas of both good Hg capturing carbons, A1 and A3, were over $100 \mathrm{~m}^{2} / \mathrm{g}$. However, the $\mathrm{Hg}$ capture capacities of A1 and A3 were higher than a specialty carbon developed specifically for $\mathrm{Hg}$ adsorption. The surface area of this specialty carbon was $570 \mathrm{~m}^{2} / \mathrm{g}$. This suggests high surface area may also not be the determining factor in good $\mathrm{Hg}$ adsorption. Similarly carbon B had higher surface area than A1 and A3 and did not adsorb Hg. However, some surface area must be required since sample A2 with a surface area less than $25 \mathrm{~m}^{2} / \mathrm{g}$ did not absorb Hg. As Figure 3.4 shows the pore volumes of A1 and A3 were substantially greater the $\mathrm{A} 2$ and $\mathrm{B}$. The greater pore volume was attributed to meso and macropores. Our previous work suggested that these pores were important in $\mathrm{Hg}$ adsorption (Rubel, et. al., 2005).

The Energy Institute (EI) at Pennsylvania State University activated thermally with steam slag samples A1 (-20+80), B (-20+80), B (soot), B (-80), and B (+20). The A1 slag which has high $\mathrm{Hg}$ adsorption capability lost $94 \%$ of its $\mathrm{Hg}$ adsorption capacity on activation. This again suggested that factors other than higher surface area and pore volume were important in $\mathrm{Hg}$ adsorption for A1. Mineral content of the slags may play an important role in $\mathrm{Hg}$ adsorption. Except for the B (-80) samples, the other B activated carbons had increased $\mathrm{Hg}$ adsorption at the 60 and $90 \mathrm{~min}$ thermal activation times. Steam activation at $90 \mathrm{~min}$ also improved $\mathrm{Hg}$ adsorption in all B slag carbons except for B (-20+80). However, the increased $\mathrm{Hg}$ adsorption was still much lower than the untreated sample A1. There were no correlations between BET $\mathrm{SA}$ or pore volumes and $\mathrm{Hg}$ adsorption capacities indicating that some SA and pore volume were required but were not the determining factors in $\mathrm{Hg}$ adsorption.

Mercury, sulfur and chlorine XAFS spectra were collected for two slag carbon samples of differing $\mathrm{Hg}$ contents, $\mathrm{A} 1$ and $\mathrm{A} 2$, and a control sample (A1 with no $\mathrm{Hg}$ ). Analysis of the $\mathrm{Hg}$ XAFS data suggested that the $\mathrm{Hg}$ is bound predominantly to $\mathrm{S}$; however, it should be noted that $\mathrm{Cl}$ is not eliminated by the data because of its proximity to sulfur in the periodic table and its similar X-ray scattering properties. In addition, the data indicated that the $\mathrm{Hg}$ coordination and bond length to sulfur were significantly reduced compared to that in cubic HgS. However, this may be a result of the mercury being bound at the surface of the sorbent rather than in a welldefined crystal structure. Sulfur and chlorine XANES spectra indicate that the sulfur and chlorine speciation between the two gasifier carbon samples were very different. There was also a slight change between chlorine in the A1 control and the $\mathrm{Hg}$ loaded A1 which suggests that chlorine may play a role in $\mathrm{Hg}$ adsorption on this slag carbon. In the A2 sample, sulfur is present mostly as metal sulfides, whereas the A1 carbon contained elemental sulfur, thiophene and sulfate forms as major forms and very little, if any, sulfur as metal sulfide. The sulfur in thiophene is not very reactive as a function of its position in the aromatic ring. Thiophene is 
present in the control A1 as well as in A1 loaded with $\mathrm{Hg}$ at similar concentrations; therefore it is doubtful that it is a major participant in $\mathrm{Hg}$ storage. Analysis of the chlorine XANES data indicated the possible presence of organochlorine compounds.

Several of the slag samples, B (-20+80), B (soot), and B (filter cake), adsorbed $40 \%$ of the $\mathrm{NO}_{\mathrm{X}}$ adsorbed by the commercial activated carbon, HGR. The adsorption of $\mathrm{Hg}$ on sample A1 $(-20+80)$ increased its $\mathrm{NO}_{\mathrm{X}}$ adsorption capacity by $27 \%$. Therefore, the use of the good $\mathrm{Hg}$ adsorbing slags for $\mathrm{Hg}$ control could have an added benefit of further reducing $\mathrm{NO}_{\mathrm{X}}$ emissions of the primary $\mathrm{NO}_{\mathrm{X}}$ emissions control technology being used by a power plant.

$\mathrm{NO}_{\mathrm{X}}$ adsorption on the slag carbons correlated with the BET SA and micropore volumes of the samples. This is consistent with work with has shown that $\mathrm{NO}_{\mathrm{X}}$ adsorption on activated carbons is mainly in micropores with the optimum pore size of approximately 11 angstroms (Kaneko, et. al., 1988). The Hg-laden A1 (-20+80) sample had an increased in SA, total pore volume and micropore volume over the A1 sample without $\mathrm{Hg}$. BET SA, total pore volume and the micropore volume increased from 109 to $199 \mathrm{~m}^{2} / \mathrm{g}, 0.068$ to $0.081 \mathrm{~cm}^{3} / \mathrm{g}$ and 0.25 to 0.34 $\mathrm{cm}^{3} / \mathrm{g}$ respectively when $\mathrm{Hg}$ was loaded onto the sample. Previous work at the CAER has suggested that partial filling of meso and macropores with $\mathrm{Hg}$ would produce some smaller spaces (micropores or equivalent spaces) for $\mathrm{NO}_{\mathrm{X}}$ adsorption (Rubel, et. al., 2005). The increase in surface area, pore volume, and micro-pore volume observed for Hg-laden A1 support this conclusion. Additionally published work has strongly suggested that $\mathrm{Hg}$ is adsorbed as $\mathrm{Hg}^{++}$ even from simulated flue gas containing only $\mathrm{Hg}^{0}$ and that it is ionically bound to the surface of the sorbent (Huggins, et. al., 2003). Our work at the CAER agrees with chemical bonding of Hg on sorbents since it is only released above $300^{\circ} \mathrm{C}$ during heating (Rubel, et. al., 2003). The ionic binding of $\mathrm{Hg}$ to the surface of pores will result in changes in the characteristics of the pores which the data shows makes the pore more suitable for $\mathrm{NO}_{\mathrm{X}}$ adsorption.

The activated samples of two gasifier slags, A1(-20+80) and B(-20+80), were also tested for their $\mathrm{NO}_{\mathrm{X}}$ adsorption capacities. It was expected that activation would increase the $\mathrm{NO}_{\mathrm{X}}$ adsorption capacities but this was not the case. Most likely this was because the increase in the SA and pore volume of these samples was mostly related to increases in mesopore volume and not micropores. This was supported by the fact that no correlations were found between $\mathrm{NO}_{\mathrm{X}}$ adsorption and BET SA, and pore volumes for the activated samples.

As a final test of the use of gasifier slag carbons for $\mathrm{Hg}$ adsorption, three of the slags, $\mathrm{A} 1$, $\mathrm{A} 2$, and $\mathrm{B}$, were tested in a simulated flue gas containing $\mathrm{NO}$ and $\mathrm{SO}_{2}$. All were compared at the same particle size distribution. As with $\mathrm{Hg}$ adsorption on these samples in air, the A1 slag out performed the other slag carbons. However, the presence of $\mathrm{NO}$ and $\mathrm{SO}_{2}$ in the adsorption gas decrease its adsorption capacity $40 \%$. The results are encouraging for the use of some gasifier carbons in the control of $\mathrm{Hg}$ emissions from electric power plants but they do indicate more work is needed to better define the interactions between $\mathrm{Hg}$ adsorption and the presence of other flue gas components.

The leaching testing of $\mathrm{Hg}$ loaded and virgin slag carbon indicated that the $\mathrm{Hg}$ captured by the slag was very stable and would not be released to the ground water on disposal. At the conditions studied less than less than $4 \mathrm{e}-4 \%$ of the $\mathrm{Hg}$ captured by the slag was released.

A cursory evaluation of the +20 mesh fraction of gasifier slag A1 and B indicated a potential for use as a pozzolanic concrete admixture and should be tested in future work.

The study of the potential use of the gasifier slag carbons indicated no obvious benefit to adding slag carbon A3 to a polymer system for the purposes of mechanical reinforcement. However, larger amounts of gasifer slag A3 positively affected the thermal diffusivity of both polypropylene and TPE-A; whereas, a large amount of A3 only made TPE-A electrically 
conductive. The addition of A3 barely affected the performance of the two polymers and, therefore, should not garner much attention as a filler material. 


\section{References}

Chang, R., 1998. "Preparation and evaluation of coal-derived activated carbons for removal of mercury vapor from simulated coal combustion flue gases," Energy and Fuels 12(6), 1061-1070

Bolger, Paul T. and Szlag, David C., 2002. "An Electrochemical System for Removing and Recovering Elemental Mercury from a Gas Stream," Environ. Sci. Technol. 36, 4430-4435

Brown, Jr., G. E., Calas, G., Waychunas, G. A., and Petiau, J., 1988. "X-ray absorption spectroscopy: applications in mineralogy and geochemistry," in: F.C. Hawthorne (ed.), Spectroscopic Methods in Mineralogy and Geology, Washington, DC, Chapter 11, 431-512.

Brunauer, S., Emmett, P., and Teller, E., 1938. "Adsorption of gases in multimolecular layers," Journal of the American Chemical Society, 60(2),309-319

Clarke, L.B. and Sloss, L.L., 1992. "Trace elements - Emissions from coal combustion and gasification,” IEA Coal Res. Rpt., IEACR/49

Cramer, S. P.,Tench, O., Yocum, N., and George, G. N., 1988. "A 13-element germanium detector for fluorescent EXAFS,” Nucl Instrum. Meth. A266,586-591

Dreizler, R.M., Gross, E.K., 1998. Density Functional Theory, Springer-Verlag, New York

Eisenberger, P. and Kincaid, B. M., 1978. "EXAFS: new horizons in structure determinations," Science 200, 1441-1447

Ghorishi, S. Behrooz, Keeney, Robert M., Serre, Shannon D., Gullett, Brian K., and Jozewicz, Wokciech S., 2002. "Development of a Cl-Impregnated Activated Carbon for Entrained-Flow Capture of Elemental Mercury," Environ. Sci. Technol. 36, 4454-4459

Hower, J.C., Trimble, A.S., Eble, C.F., Palmer, C., and Kolker, A., 1999. "Characterization of Fly Ash from Low-sulfur and High-sulfur Coal Sources: Partitioning of carbon and trace elements with particle size," Energy Sources 21, 511-525

Hower, J.C., Maroto-Valer, M.M., Taulbee, D.N., Sakulpitkakphon, T., 2000. "Mercury Capture by Distinct Fly Ash Carbon Forms," Energy \& Fuels 14, 224-226

Huggins, F. E., and Huffman, G. P., 1995. "Chlorine in coal: an XAFS spectroscopic investigation," Fuel 74, 556-569

Huggins, F. E., Huffman, G. P., Dunham, G. E. and Senior, C. L., 1999. "XAFS examination of mercury sorption on three activated carbons," Energy \& Fuels 13, 114-121

Huggins, F. E., Yap, N. and Huffman, G. P.,1999. "XAFS Investigation of mercury sorption on carbon-based and other sorbent materials," Jpn. J. Appl. Phys. (Proc. Internat. Conf. SRMS-2, Kobe, Japan) 38 (Suppl. 38-1), 588-591

Huggins, Frank E.,Yap, Nora, and Huffman, Gerald P., 1999. "XAFS Investigation of Hg Sorption on Fly-Ash," International Ash Utilization Symposium Proceedings 
Huggins, F. E.,Yap, N., Huffman, G. P. and Senior, C. L., 2003. "XAFS characterization of mercury captured from combustion gases on sorbents at low-temperature," Fuel Proc. Technol., 82(2-3), 167-196

Hwang, Jiann-Yang and Li, Zhenglong, 2000. "Control of Mercury Emissions using Unburned Carbon from Combustion By-products," US Patent 6,027,551

Kaneko, K., Nakahigashi, Y., Nagata, K., 1988. "Microporosity and adsorption characteristics against $\mathrm{NO}, \mathrm{SO}_{2}$, and $\mathrm{NH}_{3}$ of pitch-based activated carbon fibers," Carbon 26(3):327-332

Keeler, Gerald J. and Landis, Matthew S. 1994, "Standard Operating Procedure for Analysis of Mercury in Precipitation," Version 2.0 University of Michigan, Air Quality Laboratory, Ann Arbor

Koningsberger, D. C. and Prins, R., eds., 1988. X-ray absorption - principles, applications, techniques of EXAFS, SEXAFS, and XANES, Wiley, New York, NY

Kosson, D.S., H.A. van der Sloot, F. Sanchez, and A.C. Garrabrants, 2002. "An Integrated Framework for Evaluating Leaching in Waste Management and Utilization of Secondary Materials," Environmental Engineering Science, 19(3), 159-204

Lee, P. A., Citrin, P. H., Eisenberger, P., and Kincaid, B. M., 1981. "Extended x-ray absorption fine structure - its strengths and limitations as a structural tool," Rev. Mod. Phys., 53, 769-808

Lui, Wei, Vidic, Radisav D. and Brown, Thomas D., 2000. "Impact of Flue Gas Conditions on Mercury Uptake by Sulfur-Impregnated Activated Carbon," Environ. Sci. Technol. 34, 154-159

Lytle, F. W., Greegor, R. B., Sandstrom, D. R., Marques, E. C., Wong, J., Spiro, C. L., Huffman, G. P., and Huggins, F. E., 1984. "Measurements of soft X-ray absorption spectra with a fluorescent ion chamber detector," Nucl. Instrum. Meth., 226, 542-548

Mardon, S.M., and Hower, J.C., 2002. "Impact of coal properties on coal combustion by-product quality: Examples from a Kentucky power plant," International Pittsburgh Coal Conference, $19^{\text {th }}$, 23-27, Pittsburgh, paper 47-4

Rubel, A. M., Stencel, J. M., Ahmed, S. N., 1993. "Activated carbon for selective removal of nitrogen oxide from combustion flue gas," ACS, Division of Fuel Chemistry, Preprints 38(2) 726-33

Rubel, A. M., Stewart, M. L., Stencel, J. M., 1995. "Activated carbon for control of nitrogen oxide emissions," Journal of Materials Research 10(3) 562-57

Rubel, A.M., Stencel, J.M., 1997. "The effect of low-concentration $\mathrm{SO}_{2}$ on the adsorption of NO from gas over activated carbon," Fuel 76(6) 521-526

Rubel, A., Andrews, R., Gonzalez, R., Groppo, J., Robl, T, 2005. "Adsorption of Hg and NOX on coal by-products," Fuel, 84(7-8) 911-916 
Rubel, A.M., Hower, J.C., Mardon, S.M., and Perone, R., 2003. Proceeding to the 2003 International Ash Utilization Symposium, paper no. 100, Lexington, KY

Rubel, Aurora, Andrews, Rodney, Gonzalez, Rolando, Groppo, Jack, and Robl, Thomas. “Adsorption of $\mathrm{Hg}$ and $\mathrm{NO}_{\mathrm{X}}$ on coal by-products", Fuel, 2005, 84(7-8):911.

Sakulpitakphon, T., Hower, J.C., Trimble, A.S., Schram, W.H., and Thomas, G.A., 2000. "Mercury capture by fly ash: Study of the combustion of a high-mercury coal at a utility boiler," Energy \& Fuels, 14, 727-733

Serre, S.D. and Silcox, G.D., 2000. "Adsorption of elemental mercury on the residual carbon in coal fly ash," Ind. Eng. Chem. Res. 39, 1723-1730

Stern,E. A and Heald, S. M., 1979. "X-ray filter assembly for fluorescence measurements of xray absorption fine structure," Rev. Sci. Instrum., 50, 1579-1582

White, D.M., Kelly, W.E., Stucky, M.J., Swift, J.L., Palazzolo, M.A., "Field test of carbon injection for Hg control: Camden County municipal waste combustion," ORD rpt, US EPA, Washington, DC, EPA-600/R-93-181 (PB94-101540)

Wyckoff, R. W. G., 1960. Crystal Structures, Vol. 1, (2 ${ }^{\text {nd }}$ ed.) Wiley Interscience, New York. 
FINAL TECHNICAL PROGRESS REPORT

SUBMITTED TO

THE UNIVERSITY OF KENTUCKY

\section{ADVANCED GASIFICATION BY-PRODUCT UTILIZATION}

Reporting Period Start Date: September 1, 2003

Reporting Period End Date: August 31, 2006

Principal Authors: $\quad$ Dr. Harold H. Schobert

Dr. M. Mercedes Maroto-Valer

Ms. Brandie M. Markley

Dr. Zhe Lu

Report Issued Date: September, 2006

Subcontract No: 4-67392-04-201

DOE Award No: DE-FG26-03NT41795

Submitting Organization: The Energy Institute

The Pennsylvania State University

204 Research East Building

University Park, PA 16802-2398

Phone: (814) 863-1337 


\section{Disclaimer}

This report was prepared as an account of work sponsored by an agency of the United States Government. Neither the United States Government nor any agency thereof, nor any of their employees, makes any warranty, express or implied, or assumes any legal liability or responsibility for the accuracy, completeness, or usefulness of any information, apparatus, product, or process disclosed, or represents that its use would not infringe privately owned rights. Reference herein to any specific commercial product, process, or service by trade name, trademark, manufacturer, or otherwise does not necessarily constitute or imply its endorsement, recommendation, or favoring by the United States Government or any agency thereof. The views and opinions of authors expressed herein do not necessarily state or reflect those of the United States Government or any agency thereof. 


\section{Executive Summary}

With the recent passing of new legislation designed to permanently cap and reduce mercury emissions from coal-fired utilities, it is more important than ever to develop and improve upon methods of controlling mercury emissions. One promising technique is carbon sorbent injection into the flue gas of the coal-fired power plant. Currently, this technology is very expensive as costly commercially activated carbons are used as sorbents. There is also a significant lack of understanding of the interaction between mercury vapor and the carbon sorbent, which adds to the difficulty of predicting the amount of sorbent needed for specific plant configurations.

Due to its inherent porosity and adsorption properties as well as on-site availability, fly ash carbons from coal-fired combustors or gasifiers are potential mercury sorbent candidates. Furthermore, because of the increasing restricted use of landfilling, the coal industry is very interested in finding uses for these high carbon chars as an alternative to the current disposal practice. Accordingly, the work in this study focuses on expanding the characterization of three gasification char samples provided by the Center for Applied Energy Research (CAER) at the University of Kentucky, as well as thermally activating the samples and providing to the CAER activated samples for testing of their ability to uptake mercury vapor.

One of the char samples (A1) in this study was characterized prior to activation by using LOI (loss-on-ignition), proximate, ultimate, and petrographic analyses, as well as by determining the inherent mercury content of the sample as well as performing a major and minor ash chemistry analysis using ICP spectrophotometry as well as an XPS surface chemistry analysis. The porous properties of the sample, such as surface area and total pore volume, were characterized by $\mathrm{N}_{2}$ adsorption isotherms at $77 \mathrm{~K}$. The LOI value of the sample was $58.71 \%$. The proximate analyses showed that the sample had a very low moisture content of $1.90 \%$. The ultimate analyses show that the gasification char consisted mainly of carbon with very little hydrogen, nitrogen, sulfur and oxygen. The results of petrographic analysis indicated that the sample was mainly anisotropic $(60.2$ vol.\%) and was derived from different ranks of coal, including high volatile and medium volatile bituminous coals. A considerable amount of petroleum coke $(6.3 \%)$ was also observed in the slag specimen.

The amount of inherent mercury in the sample was measured at $0.04 \mathrm{ppm}$, which is relatively low compared to other char samples. The ICP ash chemistry analysis indicated very low concentrations of $\mathrm{BaO}, \mathrm{K}_{2} \mathrm{O}, \mathrm{MgO}, \mathrm{MnO}, \mathrm{Na}_{2} \mathrm{O}, \mathrm{P}_{2} \mathrm{O}_{5}, \mathrm{SrO}$, and $\mathrm{TiO}_{2}$. The sample had a relatively low concentration of $\mathrm{CaO}$. The $\mathrm{SiO}_{2}$ and $\mathrm{Al}_{2} \mathrm{O}_{3}$ concentrations measured were comparatively moderate and the amount of $\mathrm{Fe}_{2} \mathrm{O}_{3}$ determined in the sample was relatively high. Results of the XPS surface chemistry analysis show that Ti, $\mathrm{Na}, \mathrm{K}, \mathrm{Mg}, \mathrm{Ba}$, and $\mathrm{Co}$ were not found in any measurable surface concentrations. The concentration of carbon on the surface was moderate at 71.5 atomic $\%$ and the nitrogen concentration was relatively high at 0.7 atomic \%. The amount of calcium and phosphorus detected on the surface were comparatively low and oxygen, sulfur, fluorine, 
chlorine, and aluminum had relatively high values on the surface. Surface iron in the sample was comparatively high at 1.7 atomic $\%$.

The sample was tested for its ability to adsorb mercury vapor using a mercury generation/capture rig. The time to breakthrough did not occur until after 45.8 hours and the mass loading of mercury at the time of breakthrough was measured at $7.30 \mathrm{mg} \mathrm{Hg} / \mathrm{g}$ sorbent. Overall, the char sample in this study exhibited an exceptional mercury uptake capacity. In addition, the potential use of gasification chars as precursor for activated carbon was investigated. The sample was thermally activated at a steam flow rate of 2.5 $-3.0 \mathrm{~mL} / \mathrm{min}$ for 60,120 , and 180 minutes. After steam activation, the surface area was increased to $427 \mathrm{~m}^{2} / \mathrm{g}$ compared to $153 \mathrm{~m}^{2} / \mathrm{g}$ for the parent sample. The porosity of the resultant activated carbons was related to the activation conditions used. It was observed that the surface area of activated slag sample increased with activation time, and reached a maximum value at 120 minutes. Increasing the activation time over 120 minutes resulted in a decrease of the surface area.

Follow-up activation tests were performed on the gasification char (A1) as well as two additional samples, B1 and B2. The B1 sample was separated by particle size into three samples, B1(+20), B1(-20+80), and B1(-80), to examine the effect of activation on different particle sizes. The samples were activated at different times $(60,90$, and 120 minutes) at two different steam flow rates (1.22 and $2.44 \mathrm{~mL} / \mathrm{min})$. The samples were then characterized using optical microscopy, thermogravimetric analysis, scanning electron microscopy, and x-ray diffraction to examine the effect of the activation process on the particles.

In general, the activation process reduced the carbon yield in the sample and a shift from more graphitized carbon species to spinels and magnetite was observed. The sample particles were more likely to agglomerate with increased activation time and an increased steam flow rate. There was an increase in the size and shape of the pore spaces in the carbon structures as activation time clearly resulted in more connected porosity, although this may or may not cause an increase in measured surface area.

Green carbon pellet work was conducted on the three different samples using a wellestablished method. Possibly due to the extent of oxidation in the samples, solid and compact pellets could not be formed. The mixing of the carbon species and coal-tar pitch was not effective in wetting the samples with the pitch. 
TABLE OF CONTENTS

Page

Disclaimer

2

Executive Summary 3

Table of Contents $\quad 5$

$\begin{array}{ll}\text { List of Figures } & 7\end{array}$

List of Tables 9

$\begin{array}{ll}\text { 1. Introduction } & 10\end{array}$

2. Experimental Methods $\quad 12$

2.1. Sample Characterization $\quad 12$

2.1.1. Loss-on-ignition (LOI) analysis $\quad 12$

2.1.2. Proximate analysis 12

$\begin{array}{ll}\text { 2.1.3. Ultimate analysis } & 13\end{array}$

2.1.4. Petrographic analysis $\quad 13$

$\begin{array}{ll}\text { 2.1.5. Porosity characterization } & 13\end{array}$

$\begin{array}{ll}\text { 2.1.6. Inherent mercury content } & 14\end{array}$

2.1.7. Major and minor elemental ash chemistry 14

2.1.8. XPS surface chemistry $\quad 15$

2.2. Mercury Capture Studies 15

2.3. Initial Preparation of Activated Carbons 16

2.4. Characterization of Activated Carbons 17

$\begin{array}{ll}\text { 2.5. Follow-up Thermal Activation Testing } & 17\end{array}$

2.6. Characterization of Thermally Activated Samples 19

2.6.1. Thermal gravimetric analysis (TGA) 19

2.6.2. Optical microscopy (OM) 20

2.6.3. Scanning electron microscopy (SEM) 20

$\begin{array}{ll}\text { 2.6.4. X-ray diffraction (XRD) } & 20\end{array}$

2.7. Green Carbon Pellet Studies 20

3. Results and Discussion $\quad 21$

3.1. Sample Characterization $\quad 21$

3.1.1. Proximate and LOI analysis $\quad 21$

3.1.2. Ultimate analyses $\quad 21$

3.1.3. Petrographic analysis $\quad 22$

$\begin{array}{ll}3.1 .4 . \text { Inherent mercury content } & 24\end{array}$

3.1.5. Major and minor elemental ash chemistry 24

3.1.6. XPS surface chemistry 25

3.2. Mercury Capture Studies 25

3.3. Characterization of Initial Activated Carbons 25 
3.4. Characterization of Follow-up Thermally Activated Samples 28

3.4.1. Thermal gravimetric analysis (TGA) 28

3.4.2. Optical microscopy (OM) 31

3.4.3. Scanning electron microscopy (SEM) $\quad 37$

3.4.4. X-ray diffraction (XRD) $\quad 42$

3.5. Green Carbon Pellet Work 46

$\begin{array}{ll}\text { 4. Conclusions } & 46\end{array}$

5. References 49 


\section{LIST OF FIGURES}

\begin{tabular}{|c|c|c|}
\hline & Title & Page \\
\hline Figure 2.1 & A schematic of the mercury generation/capture rig. & 16 \\
\hline Figure 2.2 & Thermal activation system schematic. & 19 \\
\hline Figure 3.1 & Part of a more porous char particle that displays anisotropic textures. & 23 \\
\hline Figure 3.2 & $\begin{array}{l}\text { Low porosity char particle containing some isotropic inertinite and } \\
\text { displaying isochromatic textures. }\end{array}$ & 23 \\
\hline Figure 3.3 & $\begin{array}{l}\text { Comparison between coal-derived char particle (isotropic, blue/purple } \\
\text { and porous) with a petroleum coke particle (highly birefringent). }\end{array}$ & 24 \\
\hline Figure 3.4 & $\begin{array}{l}\mathrm{N}_{2} \text { adsorption isotherms at } 77 \mathrm{~K} \text { for the parent char sample (A1) and } \\
\text { its steam-activated counterparts at } 850^{\circ} \mathrm{C} \text {. }\end{array}$ & 26 \\
\hline Figure 3.5 & TGA profile of sample A1 $(-20+80)$ and its activated counterparts. & 28 \\
\hline Figure 3.6 & B1(+20) and its activated count & 29 \\
\hline Figure 3.7 & B1 $(-20+80)$ and its activated counterparts. & 30 \\
\hline Figure 3.8 & TGA profile of sample B1 (-80) and its activated counterparts. & 30 \\
\hline Figure 3.9 & TGA profile of sample B2 and its activa & 31 \\
\hline Figure 3.10 & es found B1(+ & 33 \\
\hline Figure 3.11 & $\begin{array}{l}\text { Plate II - T } \\
\text { cenosphere }\end{array}$ & 34 \\
\hline Figure 3.12 & $\begin{array}{l}\text { Plate I } \\
\text { cenosp }\end{array}$ & 35 \\
\hline Figure 3.13 & tures on the reactivity of & 36 \\
\hline Figure 3.14 & $\begin{array}{l}\text { or to activation at } 120 \\
\text { n. }\end{array}$ & 38 \\
\hline Figu & $\mathrm{d}$ for 90 minutes at 120 & 38 \\
\hline Figure 3.16 & ivation at 120 times (left) & 38 \\
\hline Figure 3.17 & $\begin{array}{l}\text { ated for } 90 \text { minutes at } 120 \text { times } \\
\text { ation. }\end{array}$ & 39 \\
\hline 3.18 & $\begin{array}{l}\text { SEM images of B1(-20+80) sample prior to activation at } 120 \text { times } \\
\text { (left) and } 6,000 \text { times (right) magnification. }\end{array}$ & 39 \\
\hline Figure 3.19 & $\begin{array}{l}\text { SEM images of B1(-20+80) sample activated for } 90 \text { minutes at } 120 \\
\text { times (left) and 1,000 times (right) magnification. }\end{array}$ & 40 \\
\hline Figure 3.20 & $\begin{array}{l}\text { SEM images of B1(-80) sample prior to activation (left) and activated } \\
\text { for } 90 \text { minutes (right) at } 120 \text { times magnification. }\end{array}$ & 40 \\
\hline Figure 3.21 & $\begin{array}{l}\text { SEM images of B1(-80) sample after } 120 \text { minutes of activation at } 120 \\
\text { times (left) and } 600 \text { times (right) magnification. }\end{array}$ & 41 \\
\hline Figure 3.22 & $\begin{array}{l}\text { SEM images of the B2 sample prior to activation at } 120 \text { times (left) } \\
\text { and 1,000 times (right) magnification. }\end{array}$ & 41 \\
\hline ire 3.23 & $\begin{array}{l}\text { SEM images of the B2 sample activated for } 60 \text { minutes at } 120 \text { times } \\
\text { (left) and 1,000 times (right) magnification. }\end{array}$ & 42 \\
\hline Figure 3.24 & $\begin{array}{l}\text { SEM images of a spherical particle in the B2 sample activated for } 60 \\
\text { minutes at } 900 \text { times (left) and 3,000 times (right) magnification. }\end{array}$ & 42 \\
\hline
\end{tabular}




\begin{tabular}{|l|l|c|}
\hline Figure 3.25 & XRD profiles for A1(-20+80). & 43 \\
\hline Figure 3.26 & XRD profiles for B1(+20). & 44 \\
\hline Figure 3.27 & XRD profiles for B1(-20+80). & 44 \\
\hline Figure 3.28 & XRD profiles for B1(-80). & 45 \\
\hline Figure 3.29 & XRD profiles for B2. & 45 \\
\hline Figure 3.30 & Digital photograph of a failed pellet. & 46 \\
\hline Figure 3.31 & SEM images of failed green carbon pellet particles. & 46 \\
\hline
\end{tabular}




\section{LIST OF TABLES}

\begin{tabular}{|l|l|c|}
\hline & Title & Page \\
\hline Table 2.1 & Thermal activation test matrix. & 18 \\
\hline Table 3.1 & Proximate and LOI analysis of the parent char sample. & 21 \\
\hline Table 3.2 & Ultimate analysis of the parent char sample (A1). & 21 \\
\hline Table 3.3 & $\begin{array}{l}\text { Point count analysis of anisotropic versus isotropic carbon for the } \\
\text { parent char sample. }\end{array}$ & 22 \\
\hline Table 3.4 & $\begin{array}{l}\text { ICP spectrochemical analyis reported in oxide percentages by } \\
\text { weight (as-received). }\end{array}$ & 24 \\
\hline Table 3.5 & Concentration of elements detected on powders (relative atomic \%). & 25 \\
\hline Table 3.6 & Mercury capture analysis & 24 \\
\hline Table 3.7 & $\begin{array}{l}\text { Surface areas, pore volumes and average pore width of the parent } \\
\text { char sample (A1) and its steam activated counterparts at } 850^{\circ} \mathrm{C} .\end{array}$ & 27 \\
\hline
\end{tabular}




\section{Introduction}

Coal is the most abundant fossil fuel resource in the US, and therefore it will play an even increasing role as a source of energy in the 21 st century. Coal gasification offers one of the most versatile and cleanest ways to convert the energy content of coal into electricity, hydrogen, and other energy forms. Coal gasification electric power plants are now operating commercially in the United States and in other nations, and many experts predict that coal gasification will be at the heart of the future generations of clean coal technology plants for several decades into the future. The U.S. Department of Energy's Office of Fossil Energy has turned its attention to future gasification concepts that offer significant improvements in efficiency, fuel flexibility and economics. ${ }^{1}$ The potential use of the chars produced from gasification has to be addressed.

On March 15, 2005, the U.S. Environmental Protection Agency (EPA) issued the Clean Air Mercury Rule designed to cap and reduce mercury emissions from coal-fired power plants. When the rule is fully implemented as expected in 2018, a reduction of nearly $70 \%$ is expected in annual utility mercury emissions. ${ }^{2}$

One promising way to control these emissions for compliance is by carbon sorbent injection into the flue gas of the coal-fired utilities. The annual cost of mercury control for this technology utilizing commercial activated carbons is estimated to be in the billions of dollars. Mercury concentrations in flue gas are very low (on the order of $1 \mathrm{ppb}$ by volume), flue gas is extremely complex in nature, the residence time of the carbon sorbent in the flue gas is very brief (only around $6 \mathrm{~s}$ ), and carbon sorbent selectivity for mercury is relatively poor leading to an overall need for carbon-to-mercury ratios from $1000: 1$ to $100,000: 1$ for various commercially available activated carbon sorbents. ${ }^{3}$ Moreover, there is a lack of understanding of the interaction between mercury and the carbon sorbent, also making it very difficult to predict the amount of sorbent needed for a specific plant configuration. Due to their inherent porosity and adsorption properties as well as on-site availability, fly ash carbons from coal-fired combustors or gasifiers are potential mercury sorbent candidates. ${ }^{4}$ Furthermore, because of the increasing restricted use of landfilling, the coal industry is very interested in finding uses for these high carbon fly ashes as an alternative to the current disposal practice.

Gasification chars are a potential precursor for the production of adsorbent carbons, as described here. The present global consumption of activated carbons is over 350,000 tons and it is estimated to rise 7\% annually. ${ }^{5}$ The main reason for this expanding market is the ubiquitous use of activated carbons as adsorbent materials in a broad range of increasing household, medical, industrial, military and scientific applications. Therefore, due to the expanding market for activated carbons, especially in applications related to environmental protection, such as air and water purification, new precursors are being sought. The conventional production of activated carbons consists of a two-step process that includes a devolatilization of the raw materials, followed by an activation step. In contrast, gasification chars only require a one-step activation process, since they have already gone through a devolatilization process while in the gasifier, and therefore only requires to be activated. Furthermore, the average price for a ton of activated carbon 
ranges from $\$ 500$ up to $\$ 4000^{5}$, which implies a potential 50-400 fold increase compared to the price of the ash (currently $\$ 10-20 /$ ton).

Accordingly, the work in this study focuses on expanding the characterization of three gasification char samples provided by the Center for Applied Energy Research (CAER) at the University of Kentucky, as well as thermally activating the samples and providing activated chars to the CAER for testing of their ability to uptake mercury vapor. 


\section{Experimental Methods}

\subsection{Sample Characterization}

The characterization conducted in this study involved a variety of methods that are discussed in the subsections below.

\subsubsection{LOI analysis}

The LOI content was determined according to the ASTM C 311 procedure. The analysis was conducted in duplicate. For samples commonly derived from Eastern U.S. coals, the LOI value essentially equates to carbon content.

According to ASTM C 311, a weighed sample was dried to constant weight in an oven at 105 to $110^{\circ} \mathrm{C}$ to remove the moisture in the sample. Then, the moisture-free sample was ignited to constant mass in an uncovered porcelain crucible in a muffle furnace at a controlled temperature $\left(750 \pm 50^{\circ} \mathrm{C}\right)$. After a minimum of 15 minutes heating, the percentage of LOI was calculated to the nearest 0.1, according to Equation 2.1.

Loss on ignition, $\%=(\mathrm{A} / \mathrm{B}) * 100$

$\mathrm{A}=$ loss in mass between 105 and $750^{\circ} \mathrm{C}$;

$\mathrm{B}=$ mass of moisture-free sample used.

\subsubsection{Proximate analysis}

The proximate analysis was determined using a LECO MAC-400 proximate analyzer, including moisture, volatiles, ash and fixed carbon. The value was determined by weighing the sample in a temperature- and atmosphere-controlled environment.

Before an analysis was begun, an empty crucible was placed on the turntable. The furnace was purged with nitrogen and the furnace temperature was raised to $106^{\circ} \mathrm{C}$. The sample was continuously and automatically rotated and weighed inside the furnace. Weight loss during this period could be attributed to loss of moisture in the sample. The empty reference crucible was also being weighed, and change in the weight of this crucible was used to correct the result obtained from the sample. When the corrected sample weight stopped changing, the moisture analysis was ended and moisture result was computed for the sample. Cover was placed on the crucible and the temperature was then increased to $950^{\circ} \mathrm{C}$ in a nitrogen atmosphere to drive off the volatile matter in the sample. The weighing procedure was identical to that during the moisture analysis.

When the temperature was reduced to $600^{\circ} \mathrm{C}$, the crucible cover was removed. After the temperature was increased to $750^{\circ} \mathrm{C}$, and the atmosphere was changed to oxygen, the carbon in the sample began to combust. The sample was continuously weighed as it was in the moisture analysis. When the corrected weight stabilized, the weight loss was attributed to fixed carbon, and the remaining weight was considered ash. Based on these weights, the sample's ash and fixed carbon contents were then computed. 


\subsubsection{Ultimate analyses}

The carbon, hydrogen, and nitrogen contents in the sample were measured using a LECO CHN-600 elemental analyzer, in which the determination of carbon, hydrogen, and nitrogen was made by burning a known weight of sample in pure oxygen at $\sim 950^{\circ} \mathrm{C}$ after grinding to 60 mesh $(\sim 250 \mu \mathrm{m})$ in accordance with ASTM Method D 2013. Carbon dioxide, water vapor, oxides of nitrogen, elemental nitrogen, and oxides of sulfur were possible products of combustion. Oxides of sulfur were removed with calcium oxide in the secondary combustion zone so that water vapor cannot combine to form sulfuric acid. The remaining gases of combustion were collected in a ballast volume.

For the nitrogen determination, a $10 \mathrm{cc}$ aliquot was taken. The aliquot was carried by helium into a reagent train for the removal of $\mathrm{O}_{2}, \mathrm{CO}_{2}, \mathrm{H}_{2} \mathrm{O}$ and the reduction of $\mathrm{NO}_{\mathrm{x}}$ to $\mathrm{N}_{2}$. The thermal conductivity cell was used for measuring the remaining elemental nitrogen. At the same time as the nitrogen measurement, the carbon and hydrogen infrared cells measured $\mathrm{CO}_{2}$ and $\mathrm{H}_{2} \mathrm{O}$ levels. The results for the three elements were displayed in weight percent. This method gives the total percentages of carbon, hydrogen, and nitrogen in the organic sample as analyzed, and includes the carbon in carbonates and the hydrogen in the moisture and in the water of hydration of silicates. If the moisture content is known, results can be calculated on a dry basis.

The sulfur content was determined using a LECO SC-32 and SC-132, which are microprocessor-based instruments. Sample was combusted in an oxygen atmosphere where the sulfur oxidized to $\mathrm{SO}_{2}$. After moisture and dust were removed, $\mathrm{SO}_{2}$ gas was then measured by a solid-state infrared detector. It detected total sulfur, as sulfur dioxide, continuously.

\subsubsection{Petrographic analysis}

The petrographic composition analysis was conducted using a Zeiss Universal research microscope at $800 \mathrm{X}$ magnification in reflected, white-light illumination and oil immersion. The carbon particles were grouped as isotropic and anisotropic carbon.

Before conducting the analyses, the samples were prepared by mixing them with a coldsetting epoxy resin. The specimens were then impregnated in vacuum and centrifuged to produce a density/particle-size graded sample upon hardening of the epoxy. Petrographic mounts were prepared by cutting the impregnated samples in half to expose the densitygraded particles, mixed with epoxy, and polished for microscopic examination. Samples were ground and polished using a succession of grit papers (400 and 600 silicon carbide) and alumina polishing compounds $(0.3 \mu \mathrm{m}$ on a medium nap and $0.05 \mu \mathrm{m}$ on silk).

\subsubsection{Porosity characterization}

The porosity structure was characterized by conducting $\mathrm{N}_{2}$ adsorption isotherms at $77 \mathrm{~K}$ using a Quantachrome adsorption apparatus, Autosorb-1 Model ASIT. The BET surface area was calculated from the BET equation, using the adsorption points at the relative pressures $\left(\mathrm{P} / \mathrm{P}_{0}\right) \quad 0.05-0.35$. The values reported were corrected to a mineral-free basis. The total pore volume, $\mathrm{V}_{\text {Tот }}$, was calculated using the bulk density of nitrogen at $77 \mathrm{~K}$ from the amount of vapor adsorbed at the relative pressure of 0.95 . The average pore 
diameter was calculated from the pore volume assuming the pore was cylindrical, and then, the average pore diameter was expressed as Equation 2.2.

$$
\overline{D_{n}}=\frac{4 V_{p}}{S}
$$

where

$\overline{D_{n}}=$ average pore diameter;

$\mathrm{V}_{\mathrm{p}}=$ total pore volume;

$\mathrm{S}=$ BET surface area.

The mesopore (pores 2-50 $\mathrm{nm}$ in width) and micropore (pores $<2 \mathrm{~nm}$ in width) volumes were calculated using the BJH (Barrett-Joyner-Halenda) and H-K (Horvath-Kawazoe) equations, respectively ${ }^{6}$, and the pore volumes were derived from the $\alpha_{s}$ method using a nongraphitized carbon black sample as a standard. ${ }^{7}$

\subsubsection{Inherent mercury content}

Prior to any mercury capture tests, the inherent mercury content of the sample was determined by using a cold vapor atomic absorption (CVAA) spectrophotometer according to EPA method 7470. The inherent mercury content of the fly ash is the amount of mercury contained within the sample as it was received. It indicates the magnitude of mercury that was captured naturally by the ash sample during its formation as well as any mercury from the parent coal that was not liberated during combustion or gasification.

\subsubsection{Major and minor elemental ash chemistry}

The major and minor elemental ash chemistry analysis was determined by using a Leeman Labs PS3000UV inductively coupled plasma spectrometer (ICP). Prior to analysis, the samples were ashed at $900^{\circ} \mathrm{C}$ for at least three hours. The ash was then finely ground (to smaller than $100 \mathrm{mesh}$ ) and dissolved by a lithium metaborate fusion procedure. This technique involves adding 0.1 to $0.2 \mathrm{~g}$ of the finely crushed sample to pre-weighed vials containing $1.0 \mathrm{~g}$ of lithium metaborate. The vials were gently handshaken to thoroughly mix the two components, which were then dumped into graphitic crucibles. The crucibles were then inserted into an oven pre-heated to $900^{\circ} \mathrm{C}$ and the temperature was increased to $1000^{\circ} \mathrm{C}$ for ten minutes. In the meantime, $100 \mathrm{ml}$ of a $5 \%$ nitric acid solution was pipetted into watch glass-covered Teflon beakers and a magnetic stirring bar was added to each. After ten minutes had passed and the temperature was allowed to fall to $900^{\circ} \mathrm{C}$, the beakers were placed on magnetic stirrers, the crucibles were removed from the furnace and were swirled to pick up any uncoalesced particles, the contents were dumped into the stirring beakers. The beakers were stirred for at least 15 minutes then their contents were transferred to polyethylene bottles for storage.

Once in solution, the samples were injected into the ICP analyzer. U.S.G.S. and other well characterized rock samples were analyzed simultaneously and used to calibrate the instrument. The results were obtained on an ash-basis then were converted back to the as-received basis. The major and minor elements are reported on a percent oxide (by 
weight) basis. This does not mean that pure oxides are necessarily present in the original sample, but may be present in combination with other elements.

\subsubsection{XPS surface chemistry}

Investigation into the surface chemistry of this sample was carried out by an XPS analysis using a Kratos Analytical Axis Ultra instrument and a monochromatic aluminum $(1486.6 \mathrm{eV}) \mathrm{x}$-ray source. The sample was prepared by lightly pressing the powder onto a $3 \mathrm{M}$ double-sided adhesive tape with a mortar and pestle. After loose powder was blown free from the sample with dry $\mathrm{N}_{2}$, it was transferred to a sample plate for analysis. Uniform coverage of the double-sided tape was verified using a stereo microscope.

The XPS quantification was performed by applying the appropriate relative sensitivity factors (RSFs) for the Kratos instrument to the integrated peak areas using linear background subtraction per ASTM method E 995. The RSFs take into consideration the $\mathrm{X}$-ray cross section and average mean free path of the photoelectrons. The instrumental transmission function of the spectrometer was measured for each of the separate pass energies and applied to the samples to ensure reproducible results per ASTM E 2108-00. The approximate sampling depth under these conditions is $80 \AA$. A survey scan was initially recorded for the sample to identify elements present and overall composition. The relative composition of the surface was quantified from the high sensitivity scans and the chemical state of the carbon species was determined from the charge corrected hiresolution scan of the $\mathrm{C} 1 \mathrm{~s}$.

\subsection{Mercury Capture Studies}

A mercury generation/capture rig at the University of Nottingham was utilized to test the samples in this study for their mercury uptake capacities using a Perkin Elmer PE-2380 Atomic Adsorption Spectrophotometer (AAS) operated in cold vapor mode. ${ }^{8}$ As portrayed by Figure 2.1, the schematic of the system, mercury vapor is generated by passing nitrogen through a gas bubbler containing liquid mercury (MVG1 and MVG2), where the $\mathrm{N}_{2}$ stream is fully saturated with mercury vapor. The saturated mercury vapor passes through the sample tube filled with sorbent that is maintained at $35^{\circ} \mathrm{C}$. The output stream of the sample tube is measured by the AAS and the point at which mercury vapor is present at the outlet of the sample tube is known as the breakthrough point. The time of breakthrough occurs the instant the sorbent in the sample tube ceases to capture all of the mercury vapor fed to it.

This system has been designed to ensure a continuous flow of nitrogen through the mercury vapor generators and the AAS, which minimizes fluctuations in the concentration of mercury generated. The system operates in a loop that allows the AAS to measure three different gas streams throughout the analysis as follows: (i) "zero" air from V1; (ii) mercury vapor from MVG1 and V2, which by-passes the sorbent tube; and (iii) mercury vapor from MVG2 and V3 via the sorbent tube. ${ }^{8}$ The outputs of the loop, which are continuously monitored and recorded, are the zero gas level, the baseline mercury concentration generated, and the concentration of mercury leaving the sample tube. ${ }^{9}$ 


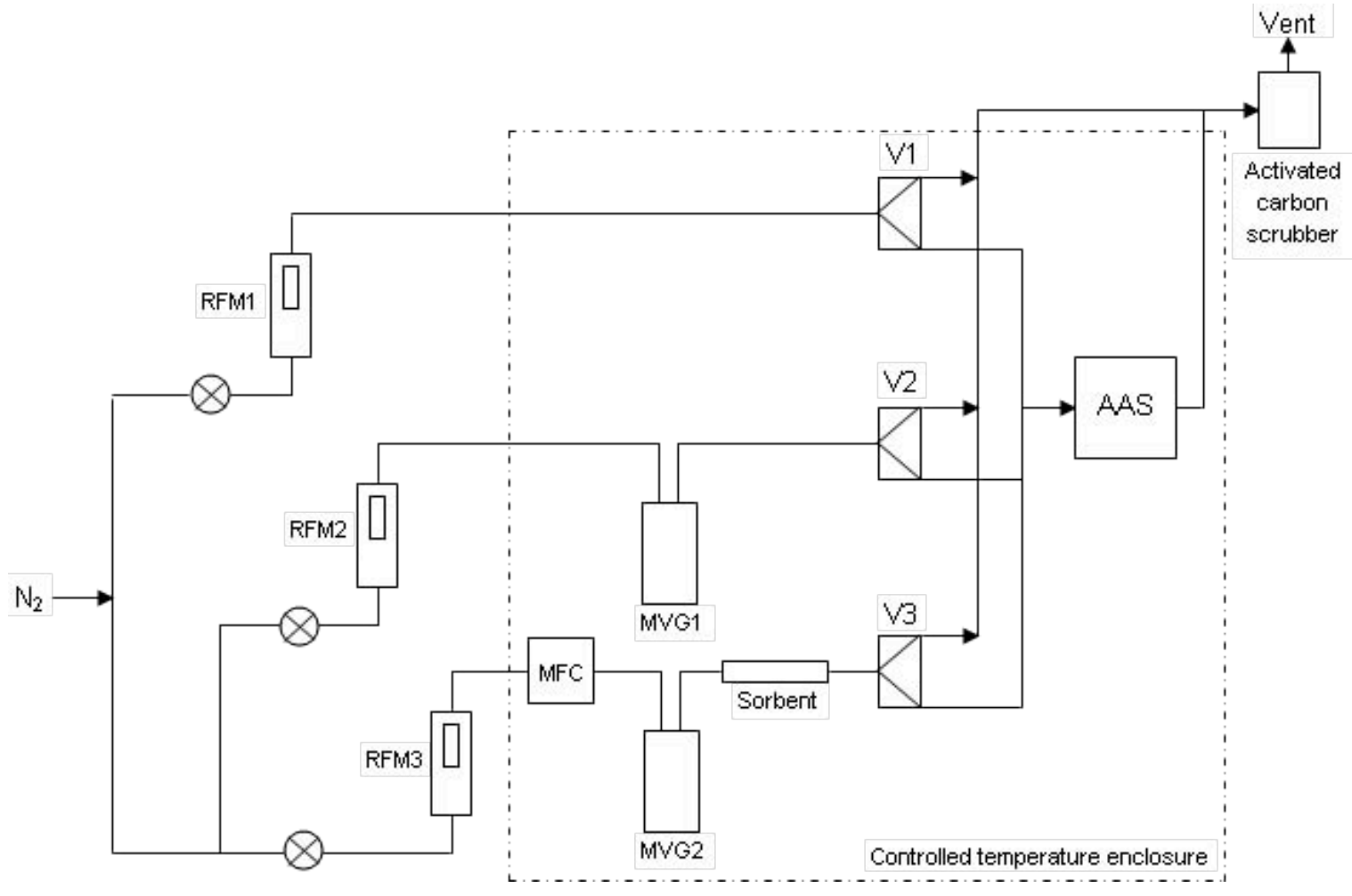

Key: $\quad$ V1 - V3 are solenoid-operated two-way valves.

MFC is a mass flow controller.

RFM1 - RFM3 are variable-area, rotameter flow meters.

MVG1 and MVG2 are identical mercury vapor generator vessels.

Figure 2.1: A schematic of the mercury generation/capture rig.

The exact concentration of mercury in the carrier nitrogen flow is calculated by Equation 2.3 , which relates mercury vapor partial pressure to temperature ${ }^{8}$. Q is the nitrogen gas flow rate $(\mathrm{ml} / \mathrm{min})$ and $C_{\text {test }}$ is the calculated mercury vapor concentration $(\mu \mathrm{g} / \mathrm{ml})$ with all volumes expressed at standard conditions of $273 \mathrm{~K}$ and $101.3 \mathrm{kPa}$. The mass loading of mercury on the sample at the time of breakthrough can be calculated by Equation 2.4, where $t_{b}$ is the breakthrough time (minutes) and $\mathrm{w}$ is the mass (g) of dry sorbent used in the test ${ }^{8}$.

$$
\mathrm{M}_{0}(\mu \mathrm{g} / \mathrm{min})=\mathrm{QC}_{\text {test }}
$$

$\mathrm{Hg}$ mass loading $(\mathrm{mg} / \mathrm{g})=\left(1000 \mathrm{t}_{\mathrm{b}} \mathrm{M}_{0}\right) / \mathrm{w}$

\subsection{Initial Preparation of Activated Carbons}

There are two principal processes for the preparation of activated carbon: physical and chemical activation. The agents used commonly in physical activation processes are 
steam and $\mathrm{CO}_{2}$, while $\mathrm{KOH}$ and $\mathrm{ZnCl}_{2}$ are used in chemical activation processes. ${ }^{10}$ In this work, steam was selected to prepare activated carbon from gasification chars utilizing two separate activation systems.

The first activation system used for the steam was a stainless steel reactor, which was heated by a horizontal furnace that has a $9 \mathrm{~cm}$ inside diameter. The versatility of the design allowed the use of different particle size, activating agent, heat treatment, residence time, flow rate of activation agent and amount of sample. Typically 2 to $3 \mathrm{~g}$ of sample was placed in the reactor that was then placed in the middle of the furnace to assure a uniform temperature zone. The furnace was then heated to the desired activation temperature under a flow of an inert gas. A thermocouple was used to monitor the temperature of the reaction zone inside the reactor. When the sample reached the desired activation temperature, a flow of steam was then introduced into the reactor. For this purpose, a HPLC pump was used to provide water at a constant flow rate of $2.5-3.0$ $\mathrm{ml} / \mathrm{min}$, and a steam generator to provide the activation agent. The steam was swept through the furnace using a flow of $\mathrm{N}_{2}$ and introduced from the bottom of the reactor to react with unburned carbon sample. During the activation process, liquid products were condensed, while gases were vented off. After a certain activation period (60-180 minutes), the flow of steam was stopped, the furnace was shut off and the reactor was quenched by $\mathrm{N}_{2}$ inside the reactor. ${ }^{11,12}$

\subsection{Characterization of Activated Carbons}

The porous properties of the produced activated carbons synthesized under controlled conditions were characterized following the same procedures described in Section 2.1.5 for the parent sample. The characterization includes the BET surface area $\mathrm{S}_{\mathrm{BET}}$, the total pore volume $\mathrm{V}_{\text {total }}$, and the micropore and mesopore volumes. The average pore width of activated carbon was calculated using Equation 2.5 under an assumption of slit-shaped pores, where $\overline{D_{n}}$ is the average pore width, $\mathrm{V}_{\mathrm{p}}$ is the total pore volume, and $\mathrm{S}$ is the BET surface area.

$$
\overline{D_{n}}=\frac{2 V_{p}}{S}
$$

\subsection{Follow-up Thermal Activation Testing}

After the initial thermal activation testing, additional samples were received and a separate activation system was utilized. The samples tested were two different gasifier chars, called A1 $(-20+80)$ and B1. Sample B1 was separated based on particle sizes into three separate samples: B1(+20), B1(-20+80), and B1(-80). The third sample, called B2, was a soot (filter cake) sample collected from the same unit as the B1 sample. The test matrix used for the activation of these samples is displayed in Table 2.1. 
Table 2.1. Thermal activation test matrix.

\begin{tabular}{lcc}
\hline \multicolumn{1}{c}{ Sample ID } & t,activation (mins) & Steam flow rate $(\mathrm{mL} / \mathrm{min})$ \\
\hline $\mathrm{B} 1(+20)$ & 60 & 1.22 \\
$\mathrm{~B} 1(+20)$ & 90 & 1.22 \\
$\mathrm{~B} 1(+20)$ & 120 & 1.22 \\
$\mathrm{~B} 1(-20+80)$ & 60 & 1.22 \\
$\mathrm{~B} 1(-20+80)$ & 90 & 1.22 \\
$\mathrm{~B} 1(-20+80)$ & 120 & 1.22 \\
$\mathrm{~B} 1(-80)$ & 60 & 1.22 \\
$\mathrm{~B} 1(-80)$ & 90 & 1.22 \\
$\mathrm{~B} 1(-80)$ & 120 & 1.22 \\
$\mathrm{~B} 2$ & 60 & 1.22 \\
$\mathrm{~B} 2$ & 90 & 1.22 \\
$\mathrm{~B} 2$ & 120 & 1.22 \\
A1 $(-20+80)$ & 60 & 1.22 \\
A1 $(-20+80)$ & 90 & 1.22 \\
A1 $(-20+80)$ & 120 & 1.22 \\
\hline B1(+20) & 90 & 2.44 \\
B1 $(-20+80)$ & 90 & 2.44 \\
B1(-80) & 90 & 2.44 \\
B2 & 90 & 2.44 \\
A1 $(-20+80)$ & 90 & 2.44 \\
\hline
\end{tabular}

The activation system, depicted in Figure 2.2, incorporated a steel reactor placed inside a vertical furnace. Approximately three grams of sample was loaded into a sample holder that was placed inside the reactor and a thermocouple was inserted to continuously monitor the sample temperature. The system was purged with $\mathrm{N}_{2}$ prior to heating and continuously throughout the heating process to create an inert atmosphere within the reactor. To begin the activation testing, the furnace was heated to $850^{\circ} \mathrm{C}$, then a flow of steam was introduced to the reactor. An HPLC pump moved water at a flow rate of either 1.22 or $2.44 \mathrm{~mL} / \mathrm{min}$ which was passed through a steam generator and then to the sample holder where activation occurred. The time of activation ( $t$, activation) was varied between 60 and 120 minutes. After this time, the flow of steam was stopped, the furnace was turned off, and the reactor was purged with $\mathrm{N}_{2}$. 


\section{Activation Unit}

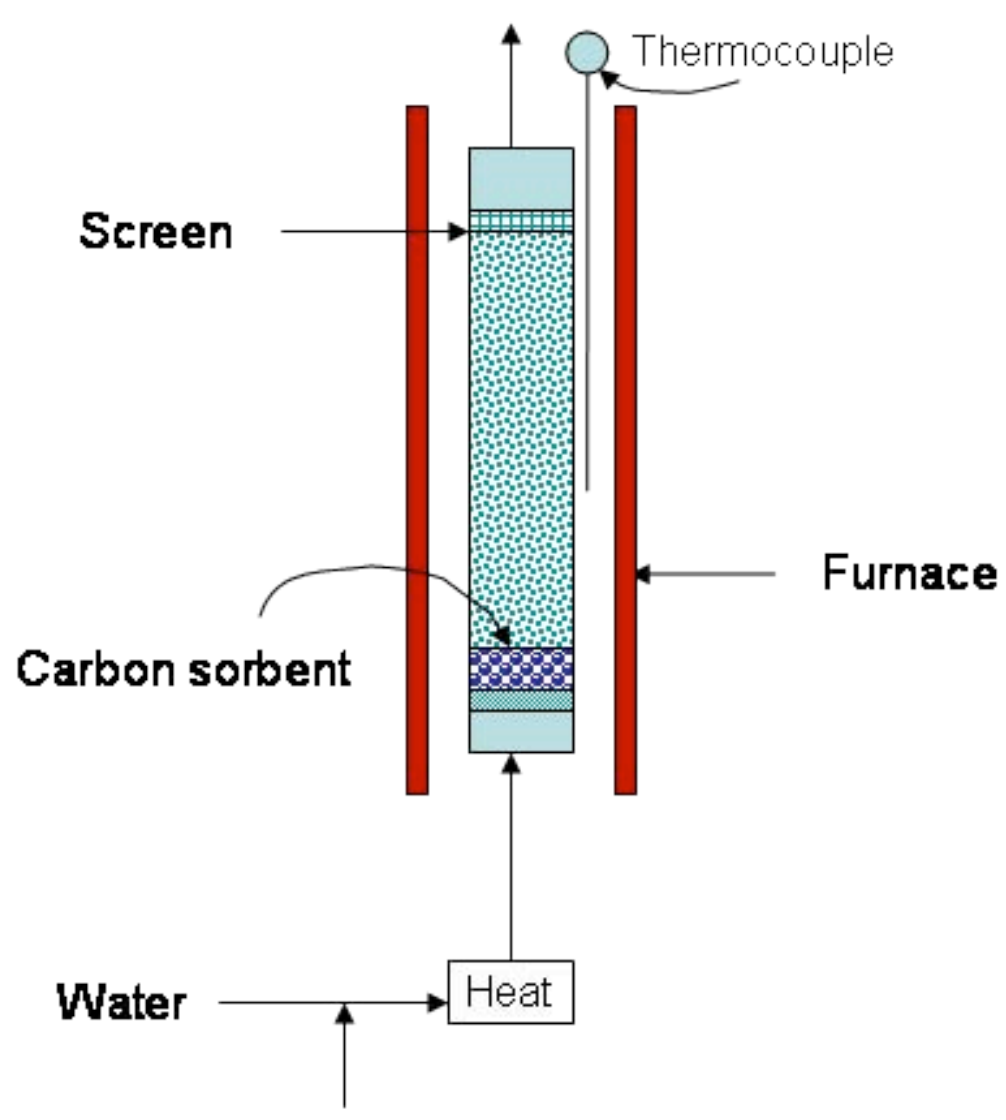

Gas Feed, $\mathbf{N}_{2}$

Figure 2.2. Thermal activation system schematic.

\subsection{Characterization of Thermally Activated Samples}

Following the thermal activation testing, the samples were characterized using various methods (optical microscopy, thermogravimetric analysis, x-ray diffraction, and scanning electron microscopy) to examine the effect the reaction had on sample structure and performance.

\subsubsection{Thermogravimetric analysis (TGA)}

A thermogravimetric analysis (TGA) was conducted using a 2050 TGA V5.4A. Approximately $15-20 \mathrm{mg}$ of each sample was heated in air flow $(100 \mathrm{~mL} / \mathrm{min})$ from room temperature to $100^{\circ} \mathrm{C}$ and then held for 10 minutes to remove moisture. The sample was subsequently heated to $900^{\circ} \mathrm{C}$ at a heating rate of $10^{\circ} \mathrm{C} / \mathrm{min}$ and the weight loss of the sample was recorded. 


\subsubsection{Optical microscopy (OM)}

An optical microscopy (OM) analysis was performed by Gareth Mitchell of the Energy Institute at Penn State University using a Zeiss Universal research microscope in polarized reflected white-light at $625 \mathrm{X}$ magnification employing a $40 \mathrm{X}$ Antiflex oil immersion objective. An AXIOCAM MRC5 digital high-resolution camera was used to take a series of photomicrographs to illustrate some of the more important observations. Approximately $0.5 \mathrm{~g}$ of sample was combined with a cold-setting epoxy resin in a plastic tube, placed in a vacuum to impregnate the pores, and spun in a centrifuge to form a gradient of particles based upon particle size and density. After hardening of the epoxy, the cylindrical samples were cut longitudinally to expose the gradient and were mounted in a mold with additional epoxy. Upon hardening the exposed gradient surface was ground and polished using a series of grit papers (320, 400 and 600 grit) and alumina polishing slurries $(0.3 \mu \mathrm{m}$ and $0.05 \mu \mathrm{m})$.

\subsubsection{Scanning electron microscopy (SEM)}

The scanning electron microscope (SEM) allows for the examination of elemental and component characterization on the micrometer to submicrometer scale. Instead of light creating an image on the surface, this focused electron beam scans the surface of the sample causing electrons to be ejected. This analysis was performed on a Hitachi HighTechnologies SEM S-3500N. Both the original (inactivated) and activated samples were examined using the secondary electron (SE) detector with magnification ranging from 120 to 8,000 times to gain information on average particle sizes and detailed surface features of each sample. A working distance of approximately 7 to $10 \mathrm{~mm}$ was used with an accelerating voltage of $20 \mathrm{kV}$ for each sample.

\subsubsection{X-ray diffraction (XRD)}

An XRD analysis was performed using a Philips X'Pert-MPD (theta-2theta) Model PW3040 diffractometer using a copper long fine focus source at power settings of $40 \mathrm{~mA}$ and $45 \mathrm{kV}$. Scans were performed from 5 to 70 degrees 2-theta with a step size of 0.02 degrees and a one second count per step. Prior to analysis, the samples were crushed using a mortar and pestle to pass through a 325 mesh sieve. The powder was mounted on a low-background quartz sample holder, which was then directly placed into the diffractometer.

\subsection{Green Carbon Pellet Work}

Green carbon pellets were formed using the gasification samples and a standard pitch in varying ratios of pitch-to-carbon, ranging from 20 to $45 \%$ pitch. Different size fractions of each sample were combined to ensure optimum mixing. Fines (-200 mesh) were $40 \%$ of the carbon part of the mixture, intermediates $(-60+200)$ were $35 \%$, and coarse particles $(-20+60)$ were $25 \%$ of the mixture. The temperature of the mold for the pellet was monitored at $110-120^{\circ} \mathrm{C}$ on a heating plate. The temperature of the pitch and carbon mixture was heated to $160^{\circ} \mathrm{C}$ while stirring continuously on the heating plate. The mixture was subsequently poured into the mold and was pressed in a hydraulic jack at a pressure of $9,000 \mathrm{lbs}$. 


\section{Results and Discussion}

\subsection{Sample Characterization}

This section presents the characterization of the Al char sample provided by the Center for Applied Energy Research at the University of Kentucky, including the LOI, proximate, and ultimate analyses, as well as surface area, and pore volume, the inherent mercury content, the ash chemistry, and the XPS surface chemistry.

\subsubsection{Proximate and LOI analysis}

Table 3.1 lists the proximate and LOI analysis of the sample on a dry basis. The proximate analyses showed that the sample had very low moisture and volatile matter contents of $1.90 \%$ and $13.07 \%$, respectively. This was due to the devolatilization undergone during the gasification process. The ash content of the unburned carbon was $36.08 \%$, and the fixed carbon content was $50.85 \%$ as obtained by difference.

Table 3.1. Proximate and LOI analysis of the A1 char sample.

\begin{tabular}{|c|c|c|c|c|}
\hline \multicolumn{4}{|c|}{ Proximate Analysis } & \multirow{2}{*}{ LOI, \% } \\
\cline { 1 - 4 } $\begin{array}{c}\text { Moisture, \% } \\
\text { (As recd.) }\end{array}$ & $\begin{array}{c}\text { Ash, \% } \\
\text { (Dry) }\end{array}$ & $\begin{array}{c}\text { V.M., \% } \\
\text { (Dry) }\end{array}$ & $\begin{array}{c}\text { Fixed carbon, } \\
\% \text { (Dry) }\end{array}$ & \\
\hline $1.90 \pm 0.16$ & $36.08 \pm 0.05$ & $13.07 \pm 0.34$ & 50.85 & $58.71 \pm 0.12$ \\
\hline
\end{tabular}

The LOI value of the sample was $58.71 \%$, which was higher than the value of fixed carbon of $50.85 \%$. This could be explained by the difference between the analytical methods used to determine LOI and proximate analyses. Fixed carbon content was calculated by subtracting moisture, volatile matter and ash from the total, i.e., $100 \%$ (ASTM D 5142-90). In contrast, when determining the LOI, the components that could decompose at the LOI test temperature $\left(750 \pm 50^{\circ} \mathrm{C}\right.$ according to ASTM C 311), such as volatile matter, and some inorganic compounds, for example, alkali salts ${ }^{13}$ were also accounted as the LOI.

\subsubsection{Ultimate analysis}

The results of ultimate analyses (dry and ash-free basis) are listed in Table 3.2. As previously reported for combustion chars, the sample consists mainly of carbon with very little hydrogen and nitrogen. ${ }^{14}$ Sulfur and oxygen were high for a char produced under gasification conditions.

Table 3.2. Ultimate analysis of the parent char sample (A1).

\begin{tabular}{|c|c|c|c|c|}
\hline $\begin{array}{c}\text { Carbon, \% } \\
\text { (Dry) }\end{array}$ & $\begin{array}{c}\text { Hydrogen, \% } \\
\text { (Dry) }\end{array}$ & $\begin{array}{c}\text { Nitrogen, \% } \\
\text { (Dry) }\end{array}$ & $\begin{array}{c}\text { Sulfur, \% } \\
\text { (Dry) }\end{array}$ & $\begin{array}{c}\text { Oxygen, \% } \\
\text { (Dry) }\end{array}$ \\
\hline $89.47 \pm 0.14$ & $0.25 \pm 0.02$ & $0.81 \pm 0.01$ & $2.80 \pm 0.02$ & 6.66 \\
\hline
\end{tabular}




\subsubsection{Petrographic analysis}

As shown in Table 3.3, about $40 \%$ of the carbonaceous fraction of the sample was isotropic carbon, largely derived from the vitrinite portion of a coal as opposed to inertinite. However, the majority of the carbon showed signs of anisotropy and was coal derived, as shown in Figure 3.1. Isochromatic areas varied from those less than $0.5 \mu \mathrm{m}$ and those less than $2.0 \mu \mathrm{m}$ to a few that were in the 4.0- 5.0 $\mu \mathrm{m}$ range, shown in Figure 3.2. Those regions less than $2.0 \mu \mathrm{m}$ are usually regarded as being high volatile A bituminous, whereas those greater than $2.0 \mu \mathrm{m}$ are usually considered to be derived from medium volatile rank coals. The majority of the char particles exhibited isochromatic areas of less than $1.0 \mu \mathrm{m}$ and were most certainly derived from a low rank high volatile A bituminous coal similar to the $0.7 \%$ (by volume) of coal found contaminating this sample.

Table 3.3. Point count analysis of anisotropic versus isotropic carbon for the A1 char sample.

\begin{tabular}{|c|c|c|c|}
\hline \multicolumn{3}{|c|}{ Anisotropic Carbon, vol.\% } & Isotropic Carbon, \\
vol.\%
\end{tabular}

A considerable amount of petroleum coke $(6.3 \%)$ was observed in the specimen that showed a concentric alignment of isochromatic regions and porosity similar to shot coke. Other particles exhibited significant porosity and could be considered a sponge coke, as shown in Figure 3.3 and Figure 3.4. The isochromatic regions were distinctively larger $(5-10 \mu \mathrm{m})$ and the carbon was much higher in reflectance than those particles derived from coal.

Pyrolytic carbon was also distinctively higher in reflectance than coal-derived and possessed isochromatic regions suggesting alignment of the carbon parallel to the depositional surface upon which it rested. This carbon is generally deposited from the cracking of gas phase hydrocarbon and carbon oxide molecules under a reducing atmosphere. It is also known to impede carbon gasification reactions by reducing the reactive surface area of the carbon upon which it is deposited. Consequently, even the low concentration of pyrolytic carbon observed in this sample (2.1\%) could have had an important influence char reactivity/burnout. 


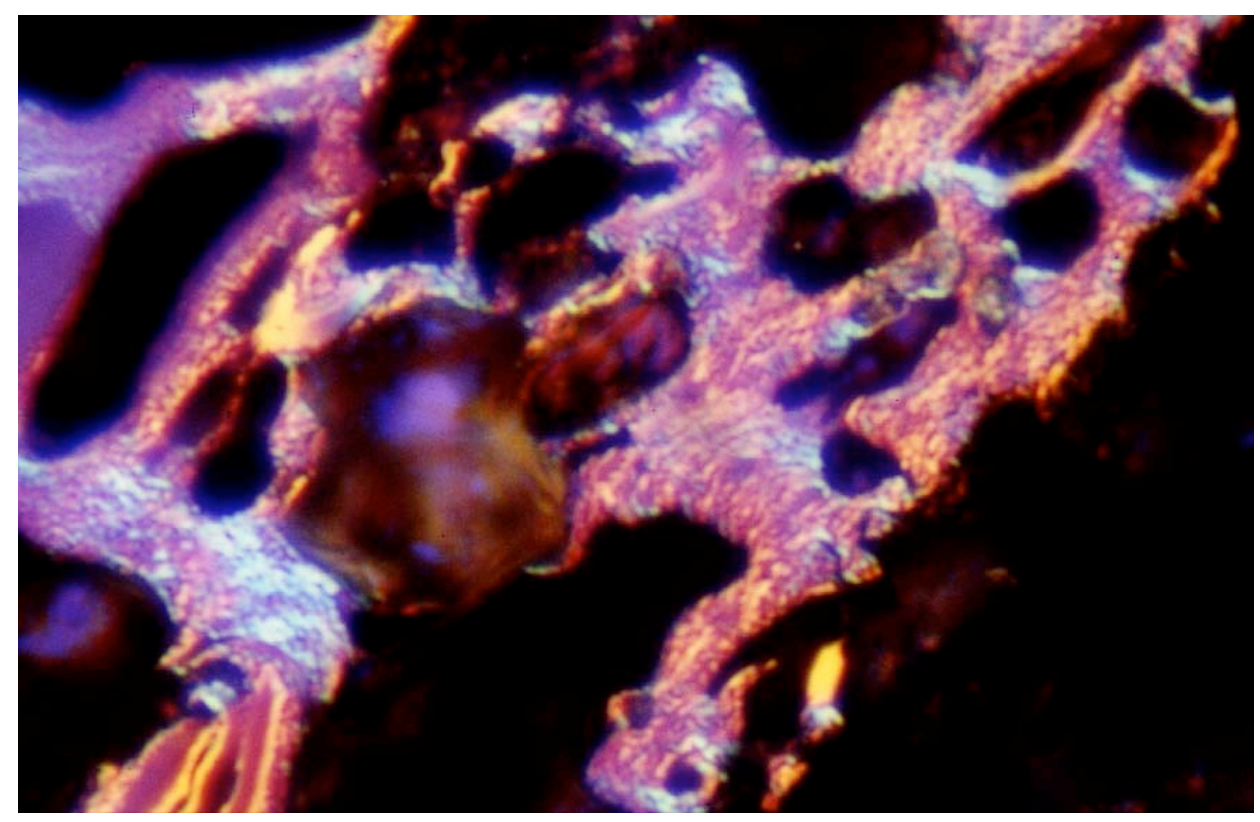

Figure 3.1. Part of a more porous char particle that displays anisotropic textures.

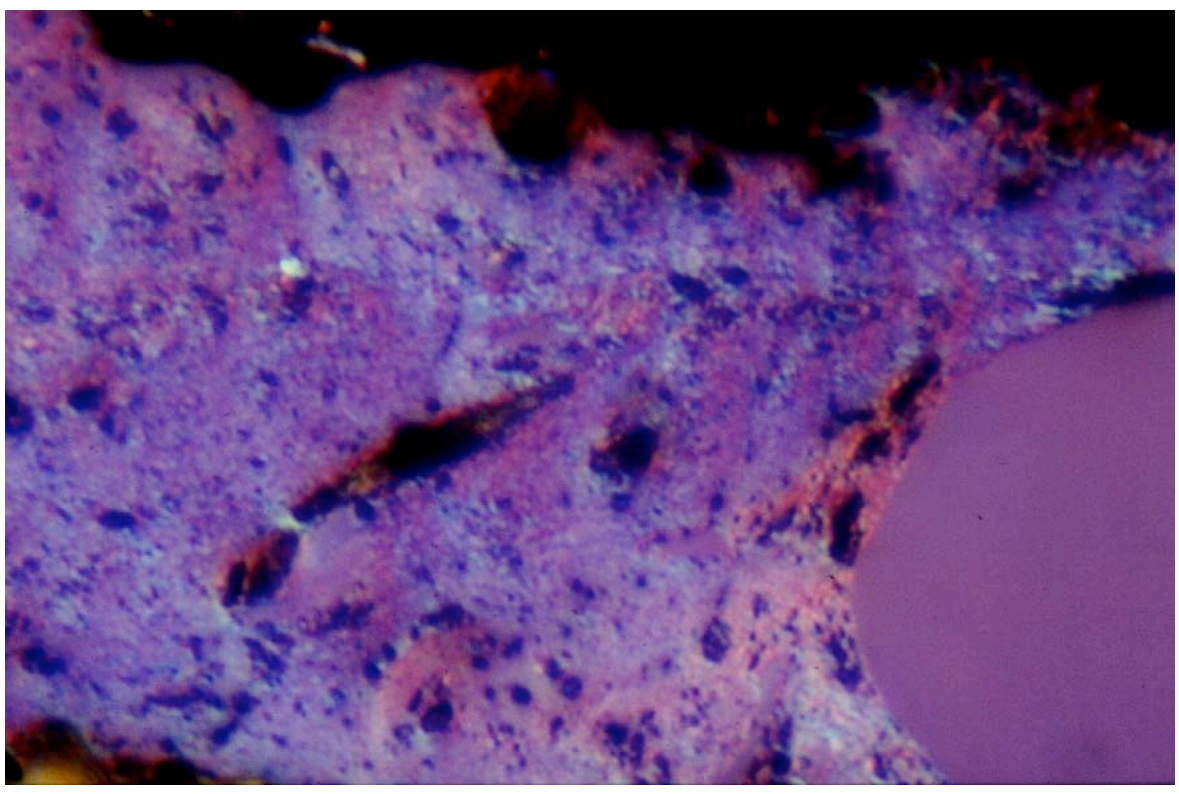

Figure 3.2. Low porosity char particle containing some isotropic inertinite and displaying isochromatic textures. 


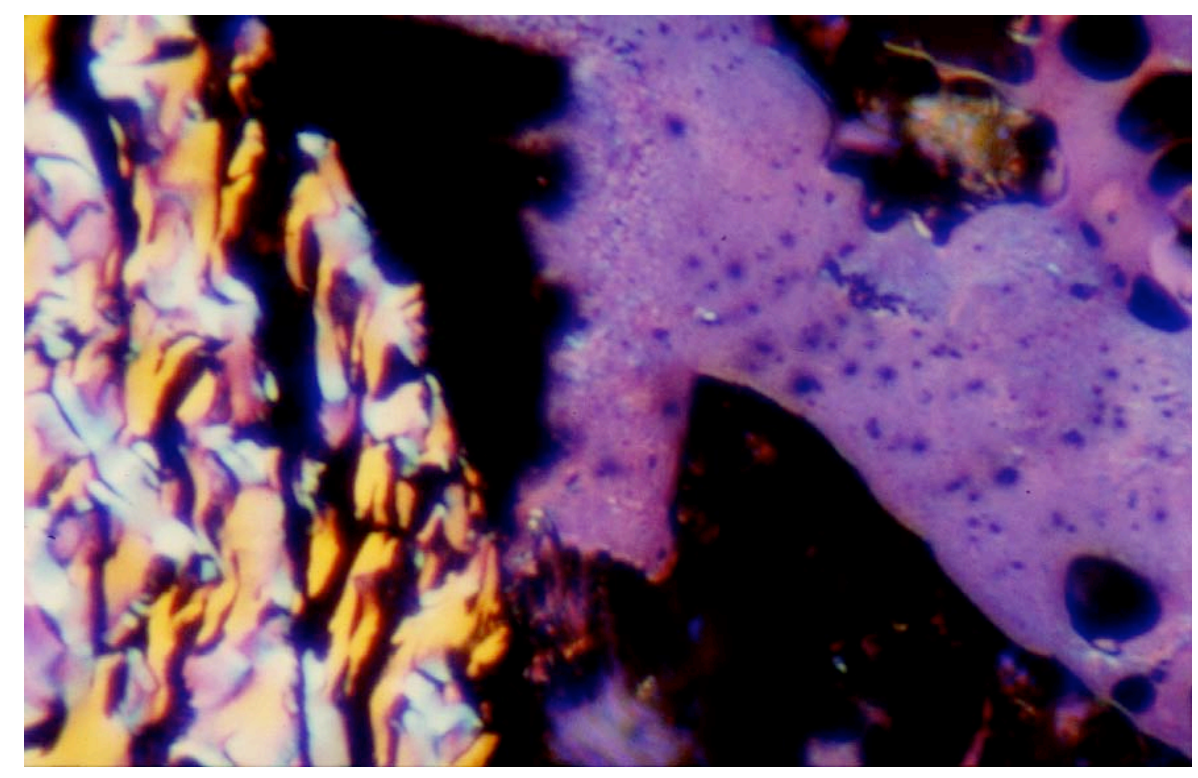

Figure 3.3. Comparison between coal-derived char particle (isotropic, blue/purple and porous) with a petroleum coke particle (highly birefringent).

\subsubsection{Inherent mercury content}

The inherent mercury content of the sample was measured using a CVAA method according to EPA method 7470. The amount of mercury contained in the sample as it was received was $0.04 \mathrm{ppm}$. Compared to other gasification samples with inherent mercury contents ranging from 0.01 to $0.11 \mathrm{ppm}^{15}$, this sample had a relatively low inherent mercury content.

\subsubsection{Major and minor elemental ash chemistry}

Table 3.4 displays the results of the ICP ash chemistry analysis for the sample. Very low concentrations of $\mathrm{BaO}, \mathrm{K}_{2} \mathrm{O}, \mathrm{MgO}, \mathrm{MnO}, \mathrm{Na}_{2} \mathrm{O}, \mathrm{P}_{2} \mathrm{O}_{5}, \mathrm{SrO}$, and $\mathrm{TiO}_{2}$ were measured. The sample had a moderate concentration of $\mathrm{Al}_{2} \mathrm{O}_{3}$ and a low concentration of $\mathrm{CaO}$ compared to other gasification samples analyzed in the same way, which ranged from $1.1 \%$ to $12.5 \% \mathrm{Al}_{2} \mathrm{O}_{3}$ by weight and $5.3 \%$ to $13.5 \% \mathrm{CaO}$ by weight ${ }^{15}$. The $\mathrm{SiO}_{2}$ concentration measured was moderate compared to other samples, ranging from $3.3 \%$ to $42.9 \% \mathrm{SiO}_{2}$ by weight. The amount of iron oxide determined in the sample was at the high end of the spectrum compared to other samples, which varied from $0.4 \%$ to $8.4 \%$ $\mathrm{Fe}_{2} \mathrm{O}_{3}$ by weight.

Table 3.4. ICP spectrochemical analyis reported in oxide percentages by weight (asreceived).

\begin{tabular}{|cccccccccccc|}
\hline $\mathrm{Al}_{2} \mathrm{O}_{3}$ & $\mathrm{BaO}$ & $\mathrm{CaO}$ & $\mathrm{Fe}_{2} \mathrm{O}_{3}$ & $\mathrm{~K}_{2} \mathrm{O}$ & $\mathrm{MgO}$ & $\mathrm{MnO}$ & $\mathrm{Na}_{2} \mathrm{O}$ & $\mathrm{P}_{2} \mathrm{O}_{5}$ & $\mathrm{SiO}_{2}$ & $\mathrm{SrO}$ & $\mathrm{TiO}_{2}$ \\
\hline 9.5 & $--^{\mathrm{a}}$ & 2.6 & 8.4 & 0.7 & 0.5 & $---^{\mathrm{a}}$ & 0.4 & 0.1 & 20.9 & 0.1 & 0.5 \\
\hline
\end{tabular}

${ }^{\mathrm{a}}$ Below instrument detection level 


\subsubsection{XPS Surface Chemistry}

Table 3.5 shows the concentration of elements on the surface of the sample as detected by an XPS analysis. The elements $\mathrm{Ti}, \mathrm{Na}, \mathrm{K}, \mathrm{Mg}, \mathrm{Ba}$, and $\mathrm{Co}$ were not found in any measurable surface concentrations. The concentration of carbon on the surface was moderate at 71.5 atomic \% compared to other gasification samples analyzed ${ }^{15}$, which ranged from 44.9 to 93.2 atomic \%. Nitrogen found on the surface of the sample was higher than other samples tested ranging from $0.1-0.4$ atomic $\%$. The surface oxygen concentration was moderate compared to other gasification samples measuring 5.7-32.9 atomic $\%$, but relatively high for a normal gasification char. The amount of calcium and phosphorus detected on the surface were low compared to other samples ranging from 0.6 -4.8 atomic \% $\mathrm{Ca}$ and $0.0-1.6$ atomic \% P. Sulfur, fluorine, chlorine, and aluminum were of moderate values on the surface. Surface iron in the sample was the highest compared to other gasification samples analyzed ranging from $0.0-1.2$ atomic $\%$.

Table 3.5. Concentration of elements detected on powders (in relative atomic \%).

\begin{tabular}{|ccccccccccc|}
\hline $\mathrm{C}$ & $\mathrm{N}$ & $\mathrm{O}$ & $\mathrm{S}$ & $\mathrm{F}$ & $\mathrm{Cl}$ & $\mathrm{Ca}$ & $\mathrm{Si}$ & $\mathrm{Al}$ & $\mathrm{P}$ & $\mathrm{Fe}$ \\
\hline 71.5 & 0.7 & 18.9 & 2.6 & 0.3 & 0.2 & 0.9 & 1.2 & 2.0 & 0.1 & 1.7 \\
\hline
\end{tabular}

\subsection{Mercury Capture Studies}

The mercury uptake capacity of the sample, A1, was determined using a mercury generation/capture rig and the results are presented in Table 3.6. The time to breakthrough indicates the length of time the sample adsorbs $100 \%$ of the mercury vapor that enters the inlet of the sample tube. The sample in this study, a gasification slag, demonstrated the best mercury capture performance compared to other gasification samples tested in the same manner, with the next best sample adsorbing only $0.023 \mathrm{mg}$ $\mathrm{Hg} / \mathrm{g}$ sorbent over 0.27 hours. ${ }^{15}$

Table 3.6. Mercury capture analysis.

\begin{tabular}{|cccc|}
\hline $\begin{array}{c}\text { W, sample } \\
(\mathrm{g})\end{array}$ & $\begin{array}{c}\mathrm{t} \text {, breakthrough } \\
(\mathrm{hr})\end{array}$ & $\begin{array}{c}\text { Hg loading } \\
(\mathrm{mg} \mathrm{Hg})\end{array}$ & $\begin{array}{c}\text { Hg loading } \\
(\mathrm{mg} \mathrm{Hg} / \mathrm{g} \text { sorbent })\end{array}$ \\
\hline 0.491 & 45.79 & 3.6 & 7.30 \\
\hline
\end{tabular}

\subsection{Characterization of Initial Activated Carbons}

The nitrogen adsorption isotherms at $77 \mathrm{~K}$ were conducted on the A1 sample and its activated counterparts using a Quantachrome adsorption apparatus, Autosorb-1 Model ASIT, as described in Section 2.1.5. The isotherms here throughout this report are presented on a carbon basis by subtracting the volume of "pure ash" from the total volume obtained from the BET measurement at $\mathrm{P} / \mathrm{P}_{0}=0.95$, and then being divided by the carbon content. In this study, the "pure ash" was obtained by completely burning the sample in the presence of air at $850^{\circ} \mathrm{C}$ (the moisture, carbon, and volatile matter were removed with only the ash being left).

Figure 3.4 shows the $\mathrm{N}_{2}-77 \mathrm{~K}$ adsorption isotherms for the A1 sample and its steamactivated carbons produced at $850^{\circ} \mathrm{C}$ for different times ranging from 60 to 180 minutes. The activated samples are named with the activation times in minutes. For example, A160 indicates that the sample was generated after 60 minutes activation. 


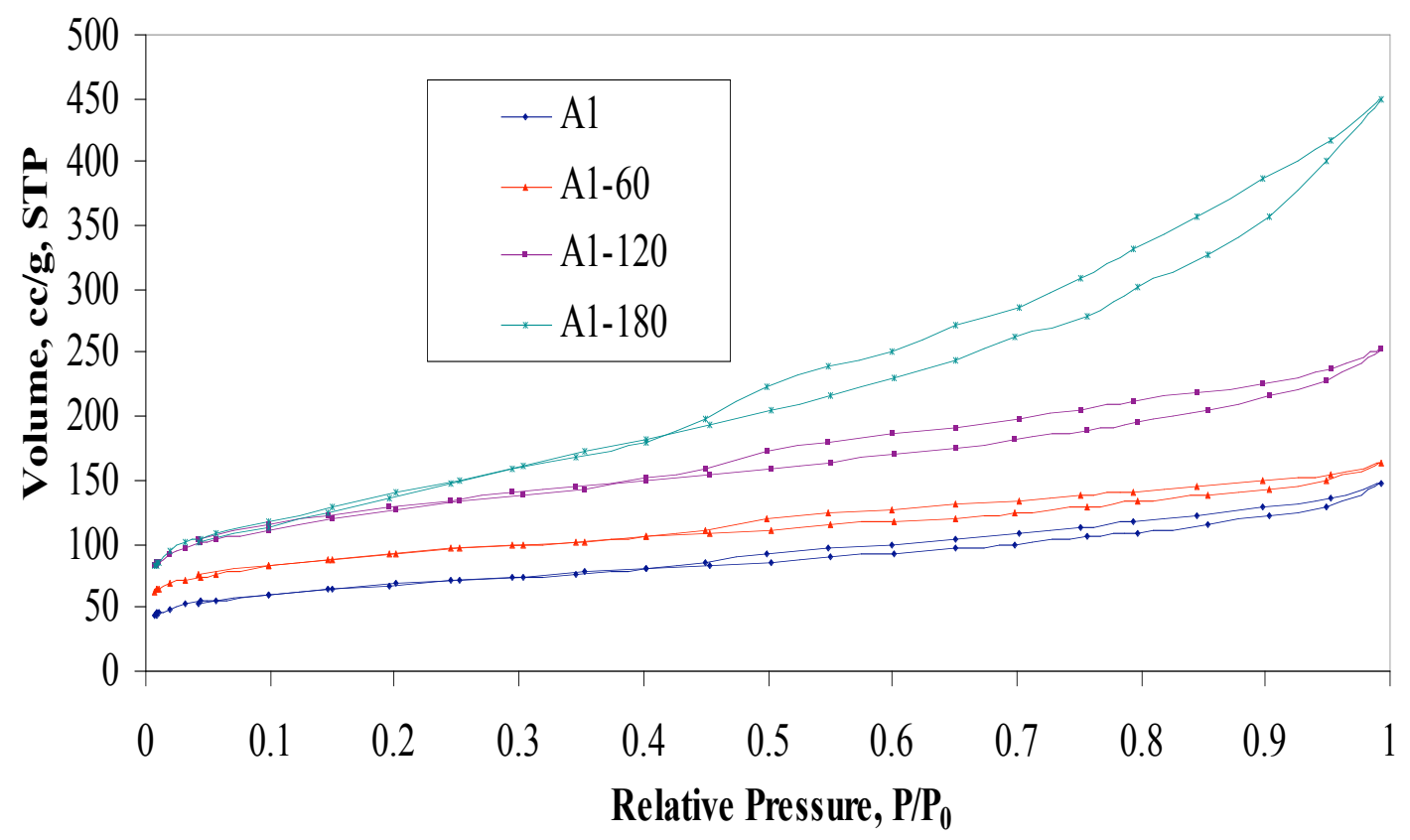

Figure 3.4. $\mathrm{N}_{2}$ adsorption isotherms at $77 \mathrm{~K}$ for the parent char sample (A1) and its steamactivated counterparts at $850^{\circ} \mathrm{C}$.

It can be observed that the parent sample (A1) presented a typical Type IV according to the Brunauer, Deming, Deming, and Teller (BDDT) classification. ${ }^{16}$ The Type IV isotherm is concave to the $\mathrm{P} / \mathrm{P}_{0}$ axis at low relative pressure, where this initial region dominates the whole isotherm and is associated with micropore filling. The isotherm then tends to level off at high relative pressure, but exhibits a hysteresis loop, which is usually associated with the filling and emptying of mesopores by capillary condensation. ${ }^{17}$ In addition, the isotherm of the parent sample presented a tail as saturation pressure was approached, indicating the presence of some macropores.

The isotherms of the activated carbons were typical Type IV, and the adsorbed volume of the isotherms increased with the activation time. There was a small increase of the adsorbed volume at low relative pressure $\left(\mathrm{P} / \mathrm{P}_{0}=0.01-0.30\right)$, and a significant increase at high relative pressure. Furthermore, there was a distinct hysteresis loop in the isotherm, which was associated with the filling and emptying of mesopores. This indicated that both micropores and mesopores were developed by extending the activation time from 60 to 180 minutes.

Table 3.7 presents the BET surface areas, total pore volumes, pore distribution, average pore diameters, and carbon yields for the parent char sample and its steam-activated counterparts after different activation times at $850^{\circ} \mathrm{C}$. The carbon yields here are calculated using the carbon weight after activation divided by the carbon weight before activation. The surface areas of the activated samples are significantly larger than the 
parent A1 sample. The surface area kept initially increases with activation time, and reaches a maximum value for sample A1-120.

Table 3.7. Surface areas, pore volumes and average pore width of the parent char sample (A1) and its steam activated counterparts at $850^{\circ} \mathrm{C}$.

\begin{tabular}{|l|c|c|c|c|c|c|c|}
\hline Sample & $\begin{array}{c}\text { Carbon } \\
\text { yield, } \\
\%\end{array}$ & $\begin{array}{c}\text { Surface } \\
\text { area, } \\
\mathrm{m}^{2} / \mathrm{g}\end{array}$ & $\begin{array}{c}\text { Vtot, } \\
\mathrm{cc} / \mathrm{g}\end{array}$ & $\begin{array}{c}\text { Vmic, } \\
\mathrm{cc} / \mathrm{g}\end{array}$ & Vmes & $\begin{array}{c}\text { Vmic, } \\
\%\end{array}$ & $\overline{D_{n}}, \mathrm{~nm}$ \\
\hline $\mathrm{A} 1$ & & 153 & 0.1410 & 0.0415 & 0.0996 & 29.4 & 3.6927 \\
\hline $\mathrm{A} 1-60$ & 80 & 294 & 0.2319 & 0.0928 & 0.1391 & 40.0 & 3.1536 \\
\hline $\mathrm{A} 1-120$ & 63 & 427 & 0.3540 & 0.1161 & 0.2379 & 32.8 & 3.3178 \\
\hline $\mathrm{A} 1-180$ & 47 & 323 & 0.3968 & 0.0377 & 0.3591 & 9.51 & 4.9344 \\
\hline
\end{tabular}

As expected, the carbon yields for the activated carbons decreased with increasing activation time, and the weight loss probably included the loss of the remaining volatile matter, which took place at the early activation stage, and the burn-off of the carbon. ${ }^{18,19}$ The change of surface area with activation time could be related to the carbon yields. In the early stages of activation, 60 - 120 minutes, the activation process led to increasing surface areas and decreasing carbon yields. When increasing the activation time to 180 minutes, the carbon yield continued to decrease while the surface area also decreased. This was probable due to pore wall consumption, and consequently formation of larger pores.

The total pore volume of the activated carbons $\mathrm{V}_{\text {total }}$ increased with the activation process $(0.1410 \mathrm{cc} / \mathrm{g}$ for $\mathrm{A} 1,0.2319 \mathrm{cc} / \mathrm{g}$ for A1-60, $0.3540 \mathrm{cc} / \mathrm{g}$ for A1-120, and $0.3968 \mathrm{cc} / \mathrm{g}$ for A1-180). The results of average pore width are also shown in Table 3.7. The average pore size $\overline{D_{n}}$ for activated carbons here is estimated using Equation 2.2. The pore width of the activated carbons decreased significantly for samples A1-60 and A1-120, compared to their precursor, due to the pore structure development during activation. The pore width increased for A1-180, probably due to the development of large pores by extensive activation.

Table 3.7 also lists the micro- and mesopore volume of the parent A1 sample and its activated counterparts. The steam activation seemed to promote the development of both micropores and mesopores with the decrease of carbon yields. This corresponded to the increase of surface areas and total pore volumes, as shown in Table 3.7. After 60 and 120 minutes activation, the micropore volume increased to about $40 \%$ and $33 \%$ of the total, respectively, compared to about $29 \%$ for the parent sample. This indicated that the development of porosity for samples A1-60 and A1-120 was mainly due to micropores, probably resulting from the opening of blocked pores of the parent sample. When the activation time was extended to 180 minutes, the micropore volume only accounted for around $10 \%$ of the total. This indicates that increasing the activation time to 180 minutes led to a widening of the porosity, mainly in the mesopore range. 


\subsection{Characterization of Follow-up Thermally Activated Samples}

Following the second round of thermal activation testing, the samples were characterized using various methods (optical microscopy, thermogravimetric analysis, x-ray diffraction, and scanning electron microscopy) to examine the effect the reaction had on sample structure and performance.

\subsubsection{Thermogravimetric analysis (TGA)}

A thermogravimetric analysis (TGA) of the three char samples is displayed in Figures 3.5 - 3.9. The profiles show the amount of weight loss in each sample as the temperature is increased to $900^{\circ} \mathrm{C}$. As expected, the original samples that have not had thermal activation treatments portray the greatest weight loss over the temperature range. In general, the samples that have been exposed to the longest activation time (120 minutes) and the higher steam flow rate $(2.44 \mathrm{~mL} / \mathrm{min})$ demonstrate lesser weight loss than those exposed to shorter activation times (60 or 90 minutes) and the lower steam flow rate $(1.22 \mathrm{~mL} / \mathrm{min})$.

The TGA profile for A1(-20+80) shows a similar weight loss curve for the samples activated for both 60 and 90 minutes with a steam flow rate of $1.22 \mathrm{~mL} / \mathrm{min}$. The parent sample (prior to activation) had the greatest weight loss and the sample activated at the higher steam flow rate $(2.44 \mathrm{~mL} / \mathrm{min})$ had the least weight loss over the temperature range.

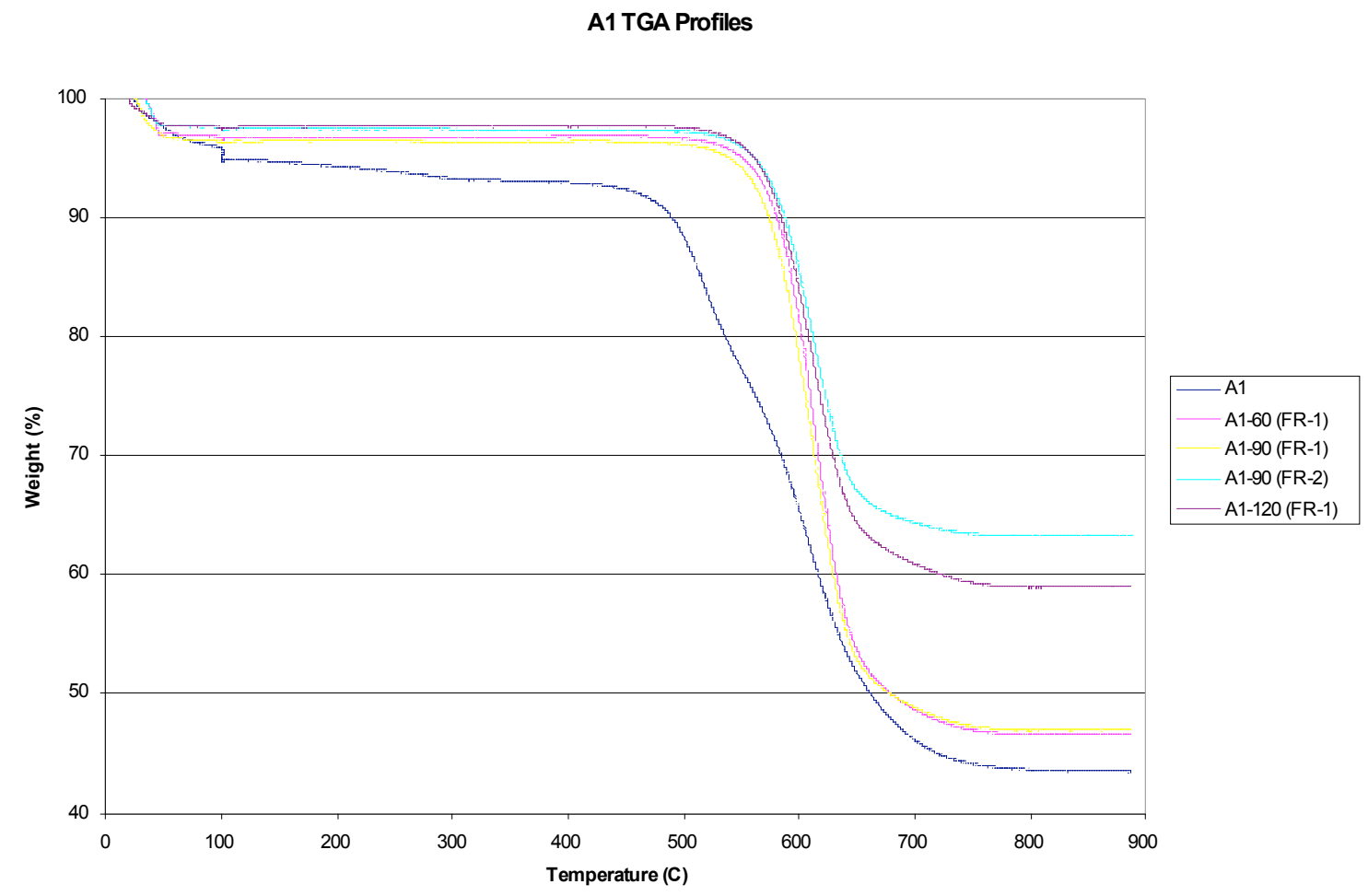

Figure 3.5. TGA profile of sample A1(-20+80) and its activated counterparts.

The TGA profiles for the sample separated by size fraction (B1) are displayed in Figures $3.6-3.8$. The largest size fraction, B1(+20), had very similar weight loss curves after 
being activated for 60 and 90 minutes. These samples even exhibited greater weight loss than the unactivated sample. This is an unexpected result as the parent sample without any heat treatment is expected to portray greater weight loss than those that have already been thermally treated. The B1(+20) sample that changed least during the increasing temperature range was the one that had been activated for 120 minutes with a steam flow rate of $1.22 \mathrm{~mL} / \mathrm{min}$.

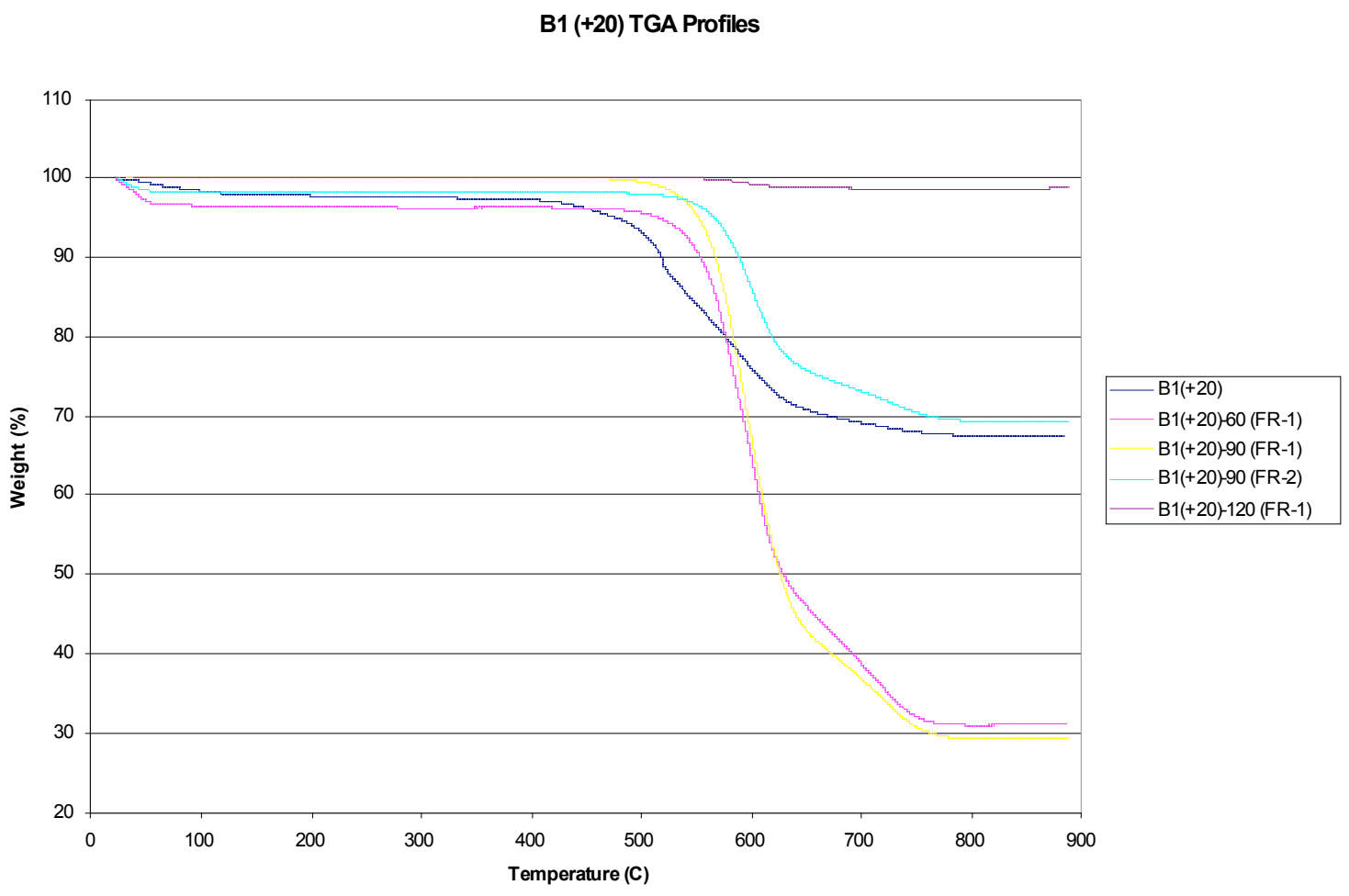

Figure 3.6. TGA profile of sample B1(+20) and its activated counterparts.

The sample B1(-20+80) displayed greater weight loss during TGA as a function of decreasing activation time, as seen in Figure 3.7. Also, the parent sample lost the most weight during exposure to elevated temperatures and the sample activated with a higher steam flow rate, lost the smallest amount of weight.

The finest portion of the sample, B1(-80), exhibited the least overall weight loss of all the samples tested. Just under $90 \%$ of the original sample remained after being exposed to temperatures of approximately $900^{\circ} \mathrm{C}$. The sample activated for the longest time $(120$ minutes) and the sample exposed to the higher steam flow rate $(2.44 \mathrm{~mL} / \mathrm{min})$ had similar weight loss curves. 


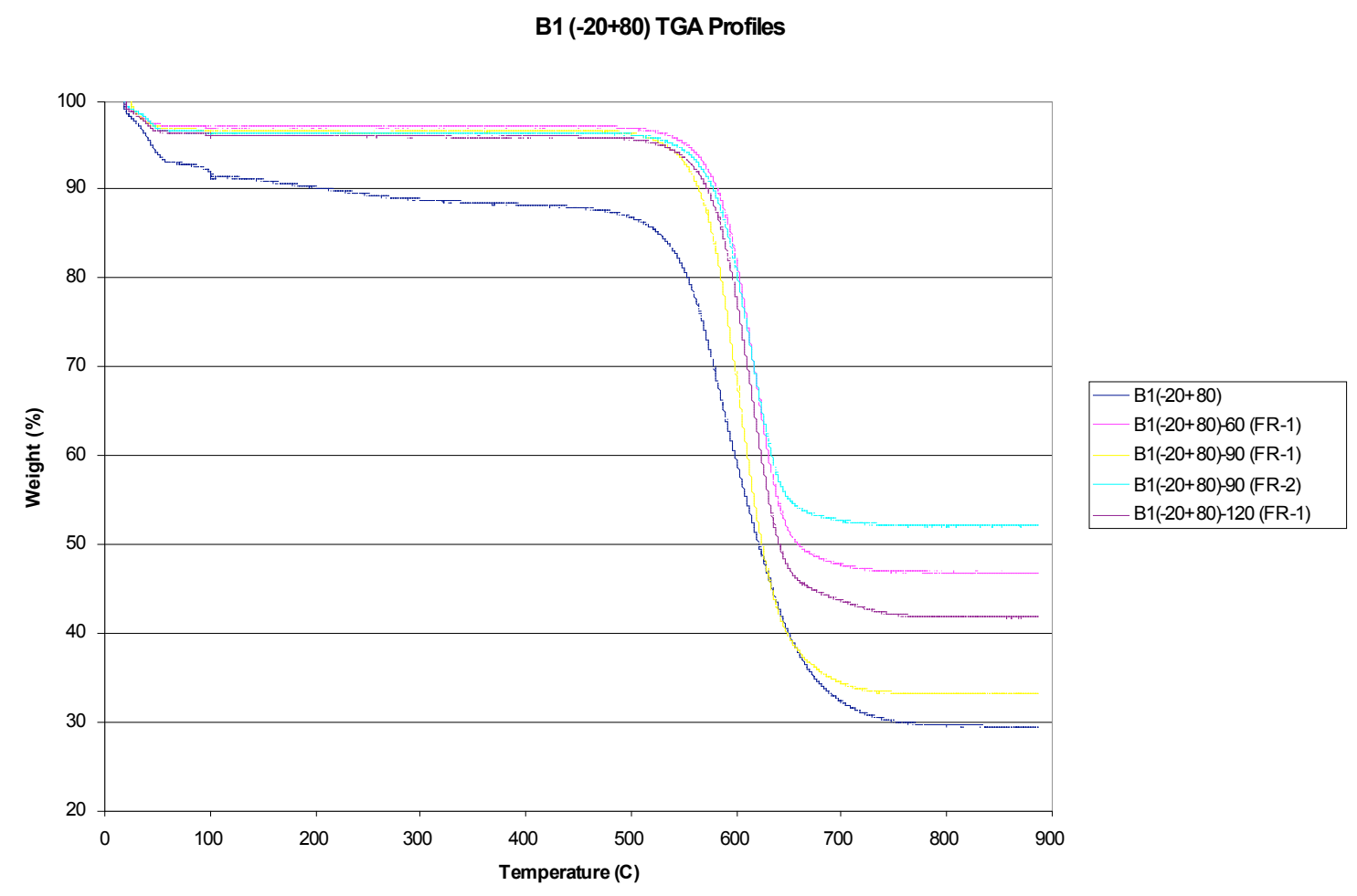

Figure 3.7. TGA profile of sample B1 $(-20+80)$ and its activated counterparts.

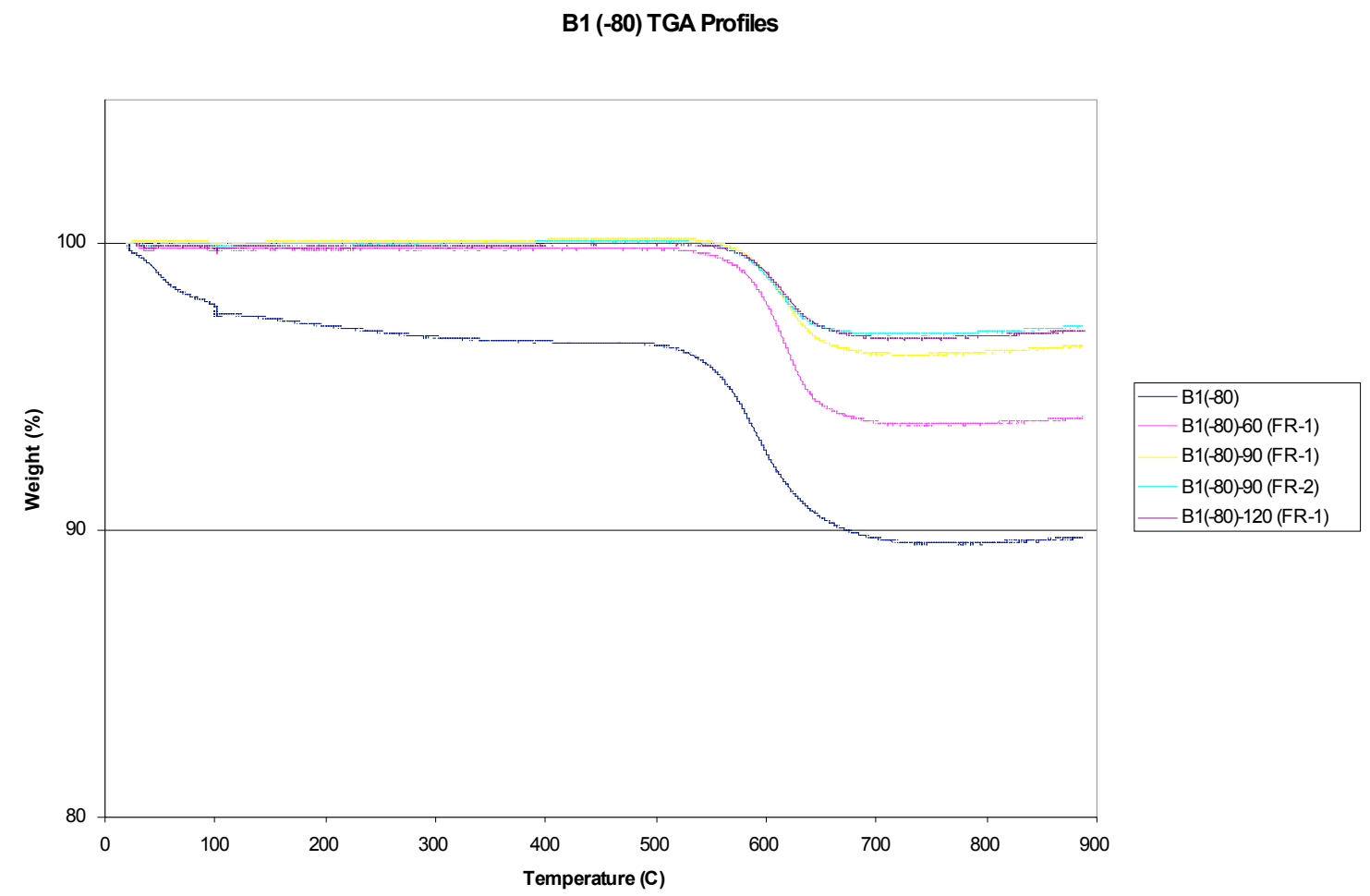

Figure 3.8. TGA profile of sample B1 (-80) and its activated counterparts. 
The soot sample, B2, is displayed in Figure 3.9. The parent sample, again displayed the greatest amount of weight loss. It is interesting to note that the least amount of weight was lost by the sample activated for 90 minutes at a steam flow rate of $1.22 \mathrm{~mL} / \mathrm{min}$. This curve is unexpected and may be due to errors during activation or during the TGA.

B2 TGA Profiles

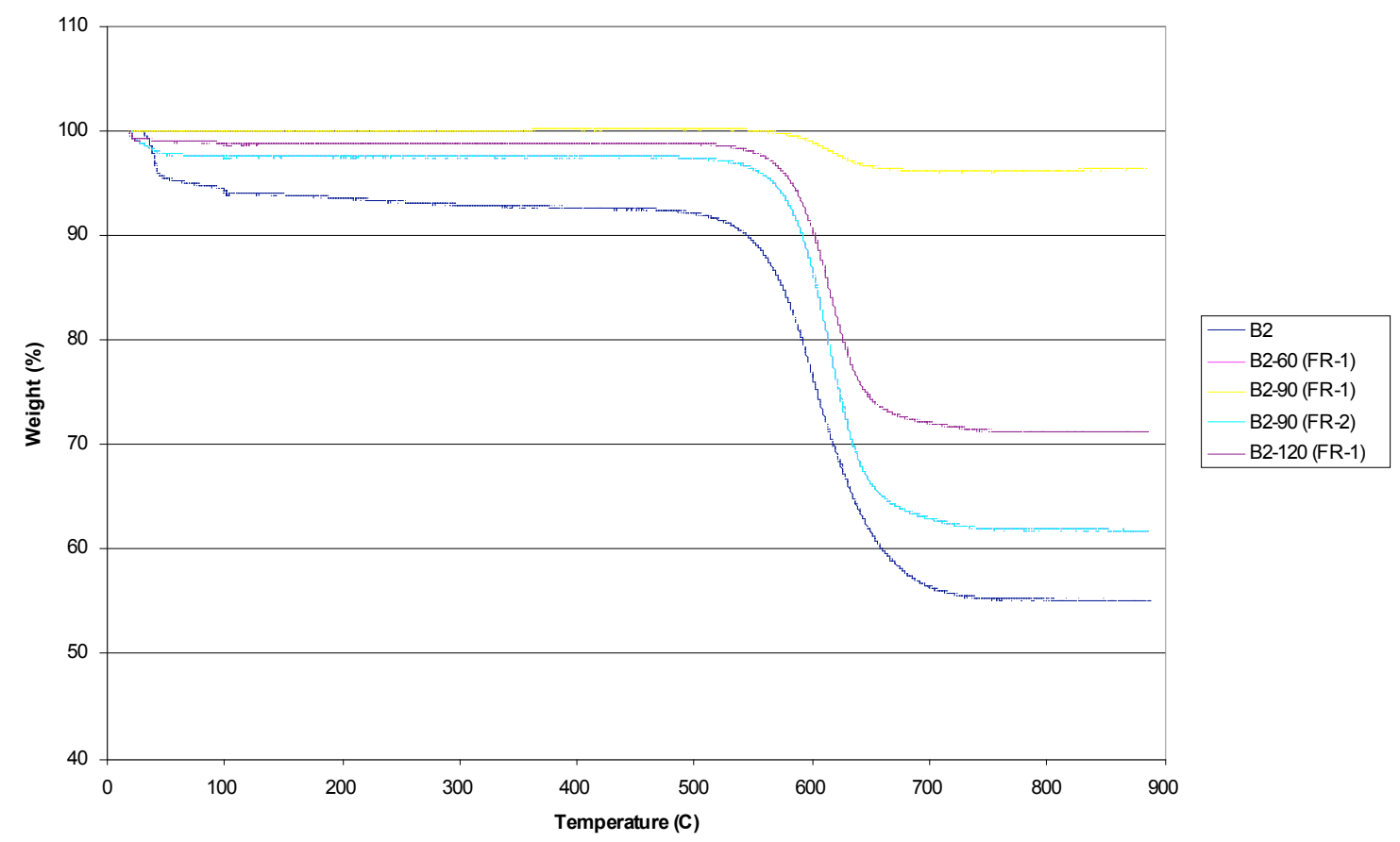

Figure 3.9. TGA profile of sample B2 and its activated counterparts.

\subsubsection{Optical microscopy (OM)}

The optical microscopy (OM) analysis showed that the samples were similar to fly ash derived from pulverized coal combustion (PCC) in that the carbonaceous fraction was composed of remnants of individual coal particles that had swollen rapidly to form cenospheres (or hollow spheres) and those that have an internal network of carbon walls. In addition, some of the original mineral matter formed solid or hollow spheres (mostly the aluminosilicates) and pyrite has formed a variety of iron oxides many of which were also spherical. However, from the optical examination, it was apparent that the particle size of the parent coal was much larger $(>1 \mathrm{~mm})$ and the heat the coal was exposed to was much lower than that typical of PCC, i.e. consistent with its gasification. Furthermore, the structure of the cenospheres and network particles and the anisotropic texture found in the carbon walls suggested that a low-rank high volatile A bituminous coal (hvAb) constituted most of the original feed material.

A typical North American coal is comprised of approximately $80 \%$ "vitrinite" (derived from the woody tissues of plants) by volume. This is also the material that becomes thermoplastic during the heating process. When it is heated at moderate to high rates with a significant oxygen presence, rapidly evolving volatile matter trapped within individual particles results in particle swelling. In addition, as coal rank increases in the 
bituminous range, an isotropic texture develops, forming semi-coke that increases in size and linearity. Coal of high volatile $\mathrm{C}$ and $\mathrm{B}$ bituminous rank develop no anisotropic texture and are isotropic even under rapid heating, whereas those of hvAb develop a texture beginning at $0.5 \mu \mathrm{m}$ (Gary, 2006). This gives a distinctive granular appearance to the carbon under polarized reflected light. As rank increases through the hvAb range the size of these textures (or isochromatic regions) increases up to 3-4 $\mu \mathrm{m}$ and begins to elongate. The size of these isochromatic textures can have an influence on carbon reactivity, as the most reactive sites to gasification are located on the edges of these tiny crystallites. Consequently, the larger their size, the less reactive surface area will be exposed to gasification or steam activation.

A small amount of evidence was found for particle agglomeration in two of the samples, $\mathrm{A} 1(-20+80)$ and $\mathrm{B} 1(+20)$ because these samples were formed from larger particles. However, the B1(+20) samples had a few very large inertinite particles in excess of 1.4 $\mathrm{mm}$ long. Inertinite does not swell during heating, so these particles may be representative of at least the largest size of the original particle size range of the feed coal.

Relatively large particle size was also observed in Plates IA, IB, and IIA in Figure 3.10 and 3.11. These photomicrographs show fairly large particles trapped in the process of becoming thermoplastic (IA) and developing anisotropic textures (IB). These result in the formation of very thick-walled cenospheres and network particles that are abundant in B1(+20) size fraction samples (IIA). Due to the refractory nature of coal, larger particles tend to develop a thermal gradient, allowing for the formation of broader areas of semicoke with smaller gas vacuoles. These thicker regions of coke have time to develop their anisotropic textures much like coals do in coke-making. Carbon walls derived from the coking of vitrinite in the $\mathrm{B} 1(+20)$ samples displayed a variety of isochromatic textures (i.e., $<0.5 \mu \mathrm{m}, 0.5-1.0 \mu \mathrm{m}$ and $1.0-3.0 \mu \mathrm{m}$ ), which were seen in different particles. There were also walls composed of isotropic carbon which suggested that perhaps more than one coal product was used in the original feed.

As demonstrated in Plate IB in Figure 3.10, the thermoplastic vitrinite formed a semicoke that sharply increases the brightness (or reflectance). Most of the vitrinite-derived carbon found in all of the samples was high-reflecting, which indicates that the temperature of gasification was at least in excess of $600^{\circ} \mathrm{C}$. When carbon is reacted at relatively high temperatures with an insufficient amount of available oxygen, the exterior surfaces of the carbon become lower in reflectance, taking on a brownish tint under the optical microscope. Many of the particles (interior and exterior surfaces) in the original samples exhibited this phenomenon and were apparently enhanced with increased duration of activation. The remnants of exposed inertinite particles, which can be identified by their lower reflectance, remnant plant cell wall structures, and isotropy, became even lower in reflectance during activation as shown in Plate ID in Figure 3.10. 



Figure 3.10. Plate I - The fate of some large coal particles found B1 $(+20)$.

Not all of the low-reflecting carbon was caused by incomplete gasification or activation, as Plate IC in Figure 3.10 shows the fate of the thermoplastic coal (seen in Plate IB) during activation for $90 \mathrm{~min}$. The high temperature used in activation has partially completed the carbonization of the once thermoplastic coal.

Photomicrographs shown in Plate II, in Figure 3.11 illustrate the influence of activation on thick-walled cenospheres with increasing residence time in the B1(+20) samples. After a 60 minute reaction time, the thinner-walled extremities become thinner and darken as seen in Plate IIB. With increasing reaction time (90 min, IIC), the interior gas vacuoles have reacted to form elongated and inter-connected pores; and by 120 mins., the more dense interiors of the wall become highly porous, as seen in Plate IID. Even though the images in Plate II in Figure 3.11 illustrate what may happen with carbon walls of similar thicknesses, a wide variety of wall structures were observed in the samples having different sizes and shapes of porosity, anisotropic textures, and mixtures of other maceral and mineral remnants. The carbon walls seen in IIA and IIB have the same anisotropic textures, whereas those found in IIC and IID were very much different. The carbon wall in IIC was mostly composed of isotropic carbon and that of IID had an anisotropic texture of about $1.0 \mu \mathrm{m}$. Carbon with larger isochromatic textures tends to be more resistant to gasification and in the +20 Eastman slag the interior of the carbon walls usually had the largest textures. Therefore, as activation time was increased these solid interior areas were found more commonly as reacted remnants in the residues. 

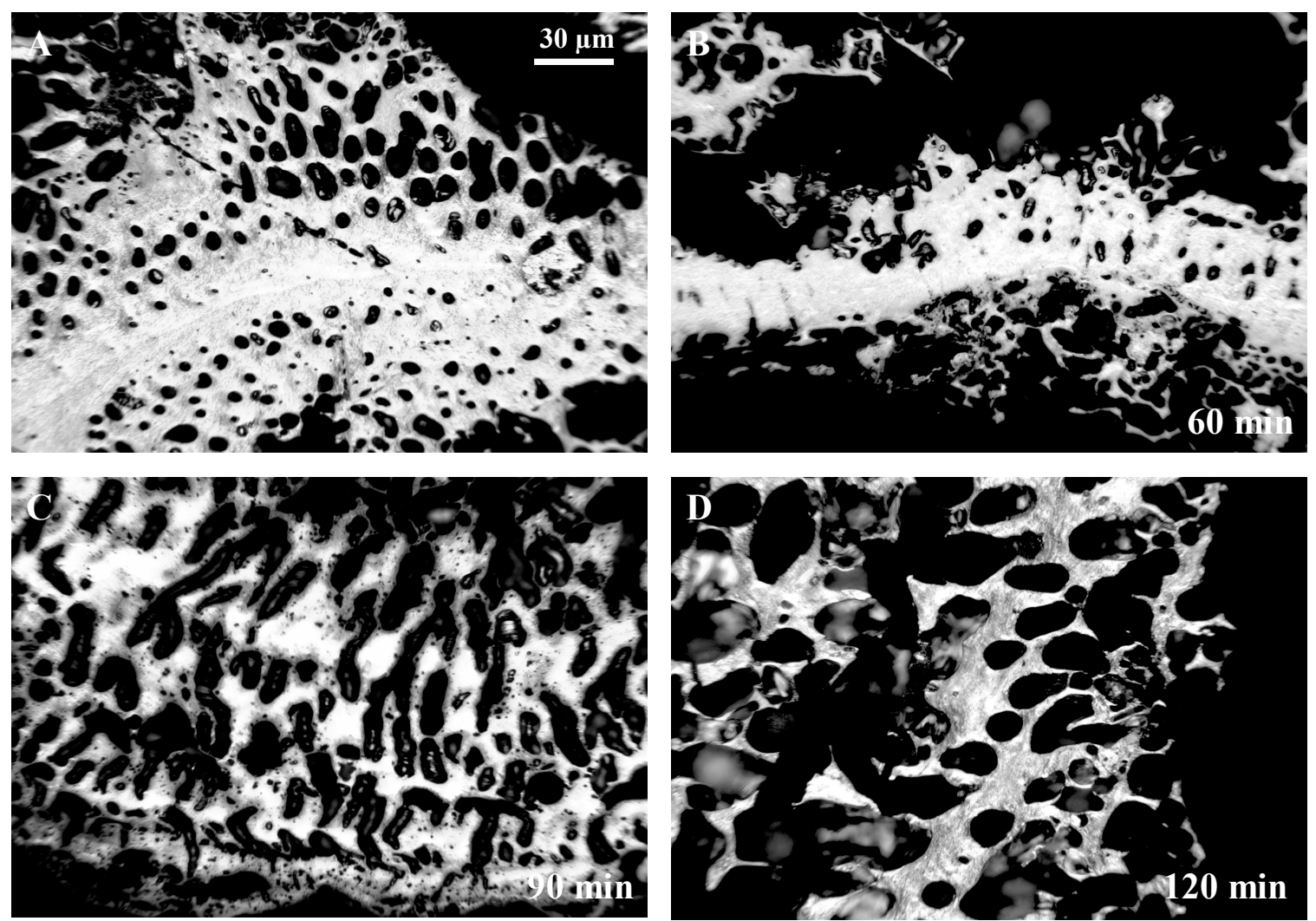

Figure 3.11. Plate II - The influence of activation time on thick-walled cenospheres and network particles found in $\mathrm{B} 1(+20)$.

The B1(-20+80) samples were composed of a mix of thin- and thick-walled structures; however, more thinner-walled cenospheres and network structures like that shown in Plate IIIA in Figure 3.12 dominated. Most of these particles have isochromatic textures smaller than $1.0 \mu \mathrm{m}$ and showed signs of oxidation, indicated by the browning of particle edges. Particle size was generally $<600 \mu \mathrm{m}$ and the rather delicate cenospheres observed intact suggested that they were formed from a specific size of coal particle. Some rather dense and angular particles containing remnants of shale partings derived from the original coal particles were observed. Furthermore, additional inertinite-derived carbon was found in this sample that remained intact and exhibited signs of oxidation. Some of the larger interior gas vacuoles within cenospheres and networks have a submicron coating of pyrolytic carbon, which is a depositional carbon resulting from the thermal cracking of carbon oxide or hydrocarbon gases. While not a substantial amount of this material was observed, coatings of pyrolytic carbon can retard the gasification and activation process of the more reactive carbon surfaces that it coats (for example, see Plate IVD in Figure 3.13).

Due to the mix of particle types in the B1(-20+80) samples, it was difficult to get a sense of the influence of activation beyond what was described for B1 $(+20)$. As activation time increased, there appeared to be more browning of edges, thinning of the wall structures, and a slight increase in the amount of larger anisotropic textures. In addition, pyrolytic carbon appeared to be slightly more common after 120 min of activation; however, it can 
not be concluded that it formed during activation. It is more likely that pyrolytic carbon was concentrated due to its relative inactivity when gasified.
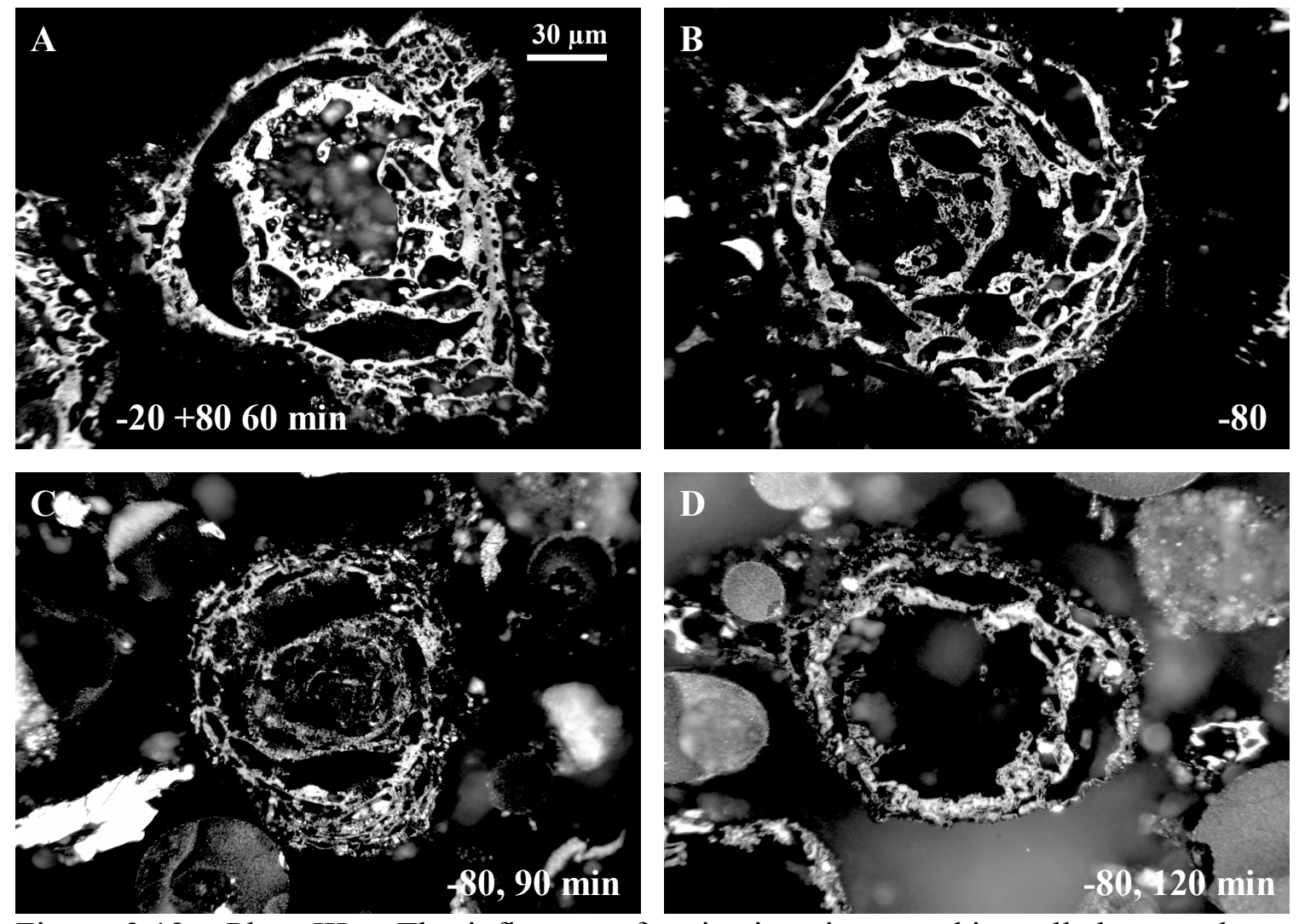

Figure 3.12. Plate III - The influence of activation time on thin-walled cenospheres found in $\mathrm{B} 1(-20+80)$.

The B1(+20) and B1(-20+80) samples contained $75-90 \%$ (by volume) of carbonaceous material. The rest of the material was composed of a variety of spheres and cenospheres of aluminosilicate particles along with a number of poorly-formed spheres of iron oxides. However, the -80 mesh fraction of B1 contained only $25-30 \%$ (by vol.) of carbonaceous material, significant fragments of broken cenospheres and network particles $(<10 \mu \mathrm{m})$. Those structures that remained intact had considerably thinner walls and were either isotropic or displayed a submicron anisotropic texture. Particles having thicker walls were thicker because of included inertinite particles or mineral matter. There were many more remnant shale particles and there were fewer individual inertinite particles.

The influence of activation is expressed in photomicrographs seen in Plate IIIB, C and D in Figure 3.12 for a specific type of thin-walled cenosphere. As activation time increased, carbon structures became thinner and lower in reflectance as a result of browning. As observed for the 90 and $120 \mathrm{~min}$. activation specimens, the estimated concentration of carbonaceous material dropped to between $10-15 \%$ (by vol.) and there were almost no $<10 \mu \mathrm{m}$ fragments observed. This change in composition could be caused by consumption or loss of the carbonaceous fraction or to sampling. 

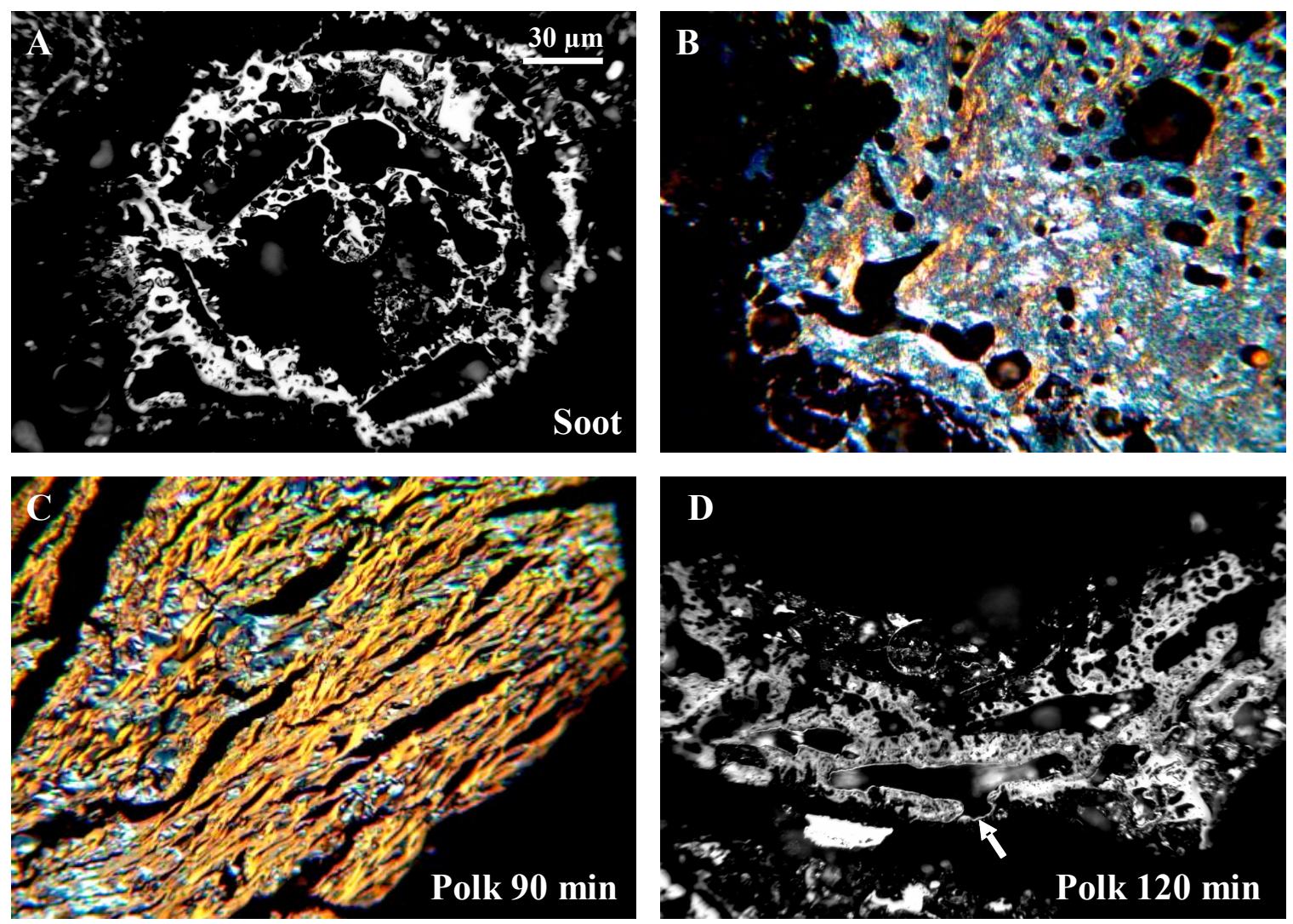

Figure 3.13. Plate IV - The influence of anisotropic textures on the reactivity of carbon structures.

In one respect, the B2 sample resembled that of B1(-80), in that it was composed of between $25-30 \%$ (by vol.) of carbonaceous material. While most of the particles were $<300 \mu \mathrm{m}$ a few approached $1.0 \mathrm{~mm}$ and there were many broken fragments $(<10 \mu \mathrm{m})$. Cenospheres, like that shown in Plate IVA in Figure 3.13 and those with much thicker walls have anisotropic textures ranging between 0.5 and $1.5 \mu \mathrm{m}$ and have a rather curious concentric arrangement of interior walls. A few such structures were seen in the other B1 samples, but they were particularly common in the B2 sample. They may have originated from a certain type of coal particle (or lithotype) having a particular mix of maceral and mineral components. Many of the thicker-walled carbon structures resulted from the inclusion of small inertinite fragments and there appeared to be more inertinite-derived carbon in this sample compared with B1(-80). A higher concentration of inorganic materials were observed and the mix was found to be about the same compared with the other B1 samples (i.e., aluminosilicate and iron oxide spheres and cenospheres), although there were many fine particles of mineral matter.

Because of the higher concentration of thin-walled cenospheres, it was difficult to characterize the influence of activation on the carbonaceous fraction. Many remained intact in the 120 min sample and their walls were thinner and lower in reflectance. Oddly, the concentration of carbonaceous material seemed to be slightly higher in the activated sample ( $35-40 \%$ by vol.), which again may be a result of sampling. 
The A1(-20+80) sample, while displaying many of the same attributes of the B1 and B2 samples just described, appeared to have been derived from different feed materials. Volumetrically, the carbonaceous fraction was estimated to be $50-60 \%$ of the specimen and composed of fairly large thick-walled cenospheres and network structure. Most of the carbon displayed anisotropic textures of $<1.0 \mu \mathrm{m}$, but individual particles having 2 - 4 $\mu \mathrm{m}$ elongated isochromatic region like that shown in Plate IVB in Figure 3.13 were observed. These regions were formed from coal particles of slightly higher rank than those observed in the B1 and B2 samples. In addition, a small amount of petroleum coke was found in the sample (see Plate IVC in Figure 3.13), some having been incorporated into coal-derived network structures, demonstrating their presence in the original feed material. In addition, a number of small unreacted coal particles were observed in the specimen and were presumed to be contamination. The inorganic constituents of A1($20+80$ ) were roughly the same as seen in B1 and B2, aluminosilicate spheres and cenospheres, a variety of iron oxides, and a significant amount of the inorganic was associated with the carbonaceous fraction.

The influence of activation was found to be less pronounced in the 60 min sample particularly with the thicker, less-porous regions of carbon. There may have been a slightly lower reflectance along some particle edges, but this was difficult to quantify. In contrast, those samples activated to 90 and 120 mins showed a significant increase in the amount of carbon having larger isochromatic regions, whereas there was a change in the isotropic carbon texture; having low reflectance owing to browning and more connected porosity. This is illustrated in Plate IVD in Figure 3.13 in which a highly tattered particle showing mostly low reflecting carbon is presented. The regions of higher reflectance have better developed isochromatic regions. Pyrolytic carbon also can be observed coating the interior of a gas vacuole, thus demonstrating its relative inactivity to the activation procedure.

\subsubsection{Scanning electron microscopy (SEM)}

Each sample was examined at 120 times magnification to get a feel for the particle sizes

and shapes. Surfaces of interest were then examined at stronger magnification (usually $\geq$ 1,000 times). Samples A1 $(-20+80)$ and B1 $(+20)$ were analyzed prior to activation and after being activated for 90 minutes with a steam flow rate of $1.22 \mathrm{~mL} / \mathrm{min}$. The SEM images from A1 $(-20+80)$ are depicted in Figure 3.14 and 3.15. After 90 minutes of activation, smoother, more agglomerated particles are present at 120 times magnification. The surface of the particles is still scattered with tiny spherical particles (most likely cenospheres and aluminosilicate spheres as discussed in Section 3.4.2). 

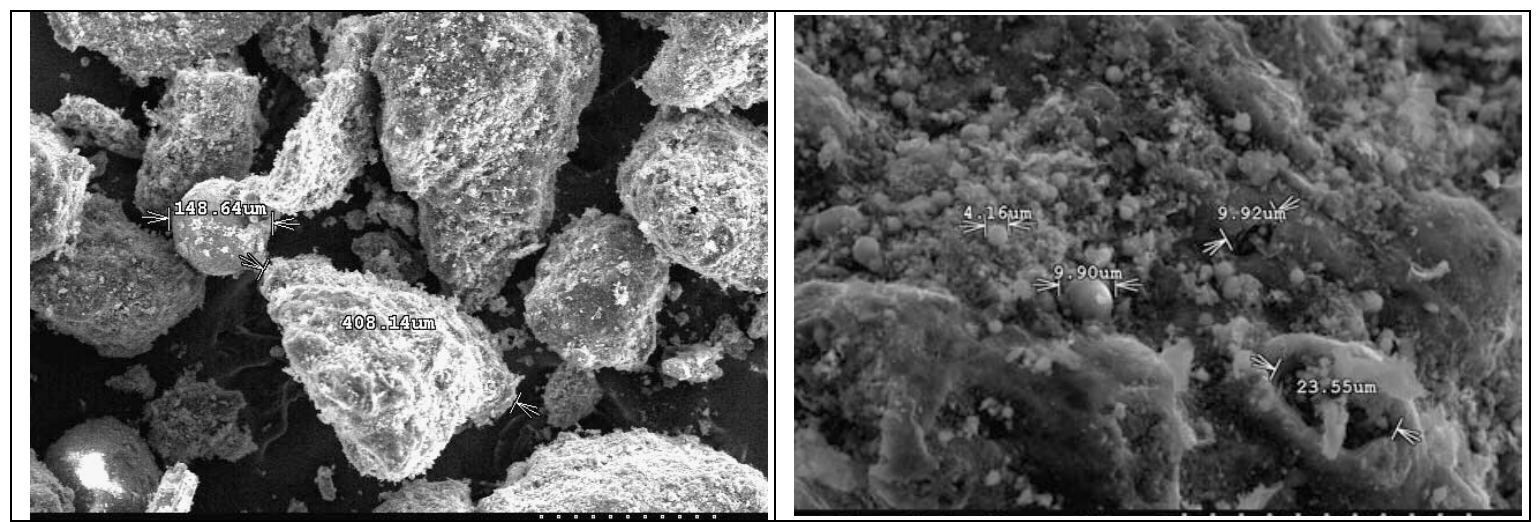

Figure 3.14. SEM images of original A1(-20+80) sample prior to activation at 120 times (left) and 1,000 times (right) magnification.
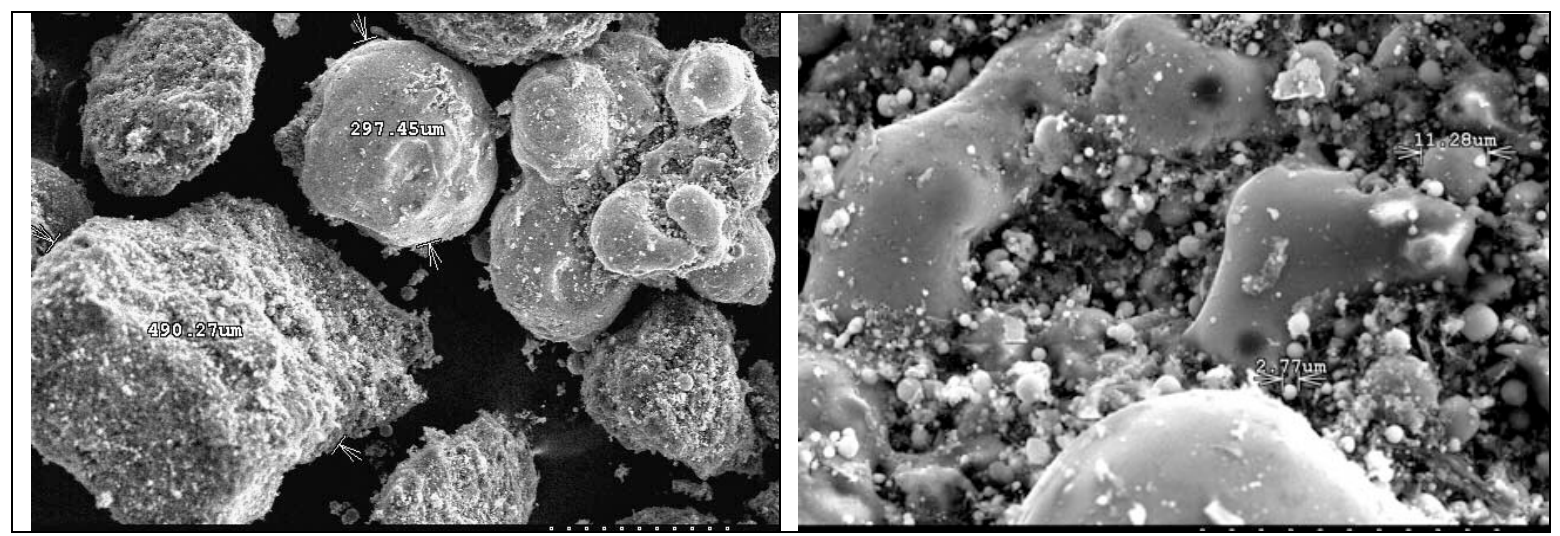

Figure 3.15. SEM images of A1(-20+80) sample activated for 90 minutes at 120 times (left) and 1,000 times (right) magnification.

The SEM images for B1(+20) are depicted in Figures 3.16 and 3.17. After activation for 90 minutes, the surface of the very large particles appears somewhat smoother with more structured, orderly micropores (as can be seen in the 1,000 times magnification images).
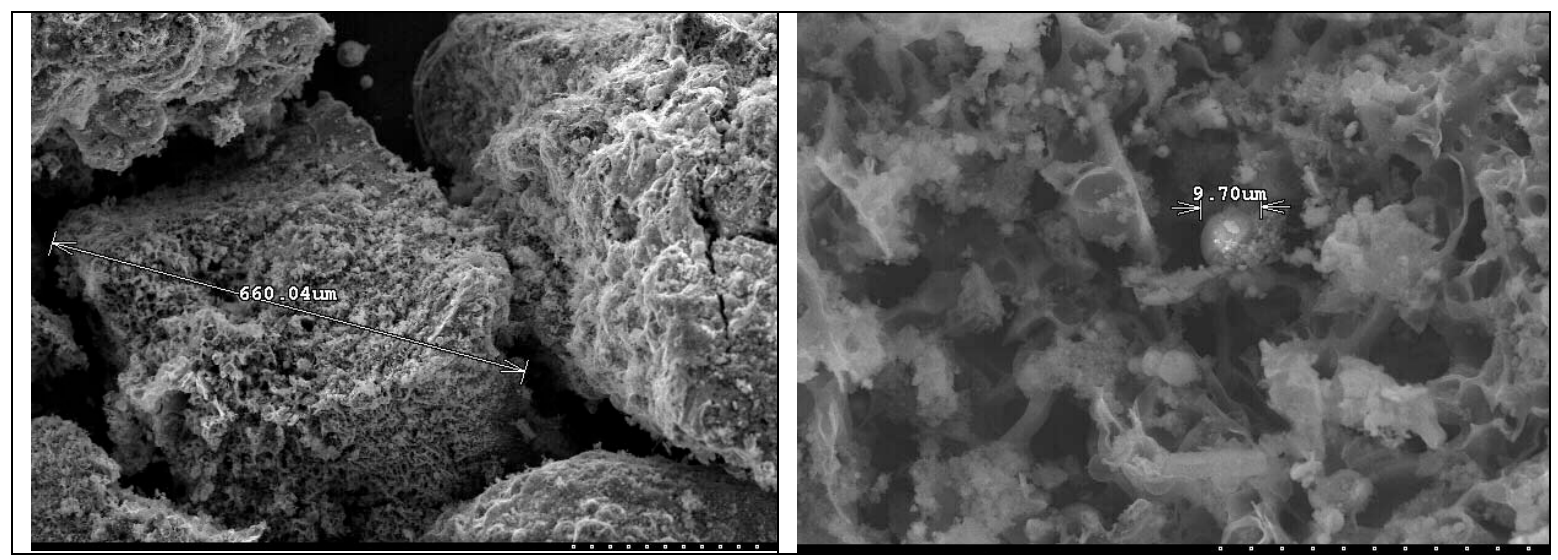

Figure 3.16. SEM images of B1(+20) sample prior to activation at 120 times (left) and 1,000 times (right) magnification. 


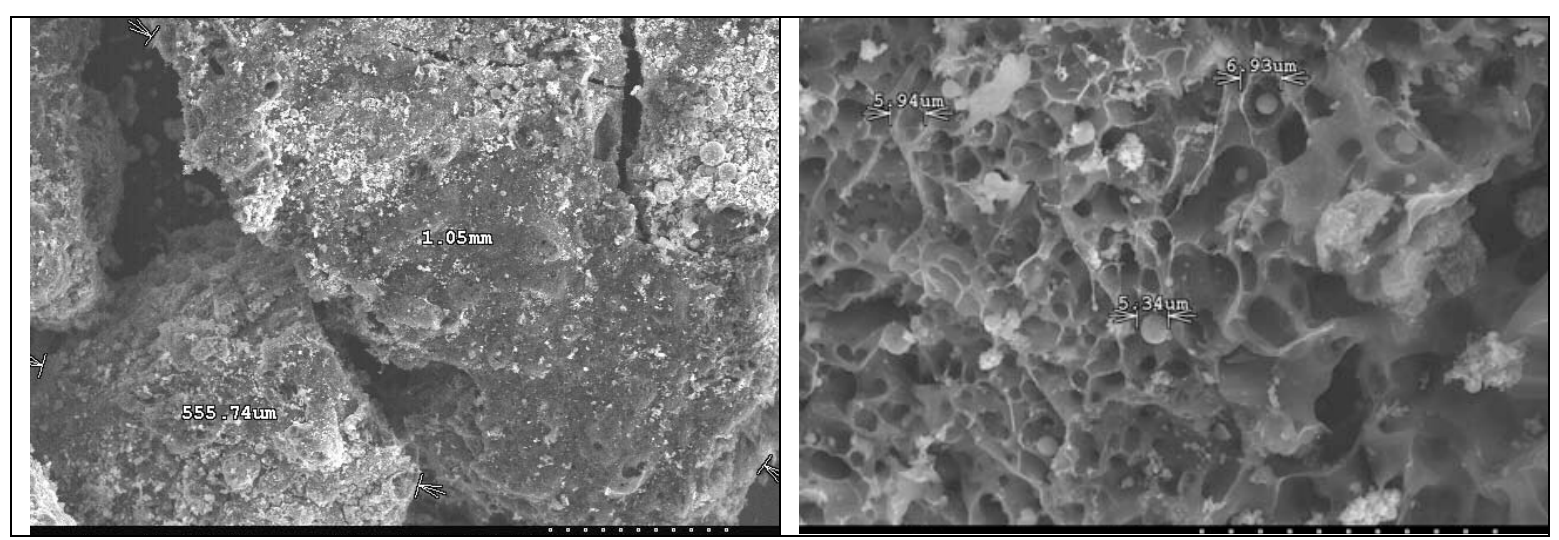

Figure 3.17. SEM images of B1(+20) sample activated for 90 minutes at 120 times (left) and 1,000 times (right) magnification.

The SEM images for B1(-20+80) are depicted in Figures 3.18 and 3.19. There is a slight difference in particle surface as can be seen at the lower magnification. After 90 minutes of activation, the surfaces of the intermediate-sized particles appear to have more crevices and more micropores. As can be seen from the 6,000 times magnified image in Figure 3.18, the surface of the inactivated particles is covered with ordered pores filled with cenospheres and other hollow or solid spheres, which are less apparent after activation. After the activation process, the surface is more crater-like, with shallower pores (micropores).

This sample was also examined after being activated for 90 minutes with a faster steam flow rate $(2.44 \mathrm{~mL} / \mathrm{min}$ vs. $1.22 \mathrm{~mL} / \mathrm{min})$. The particle sizes, shapes, and surface features were very similar to those observed for the sample with a slower steam flow rate.

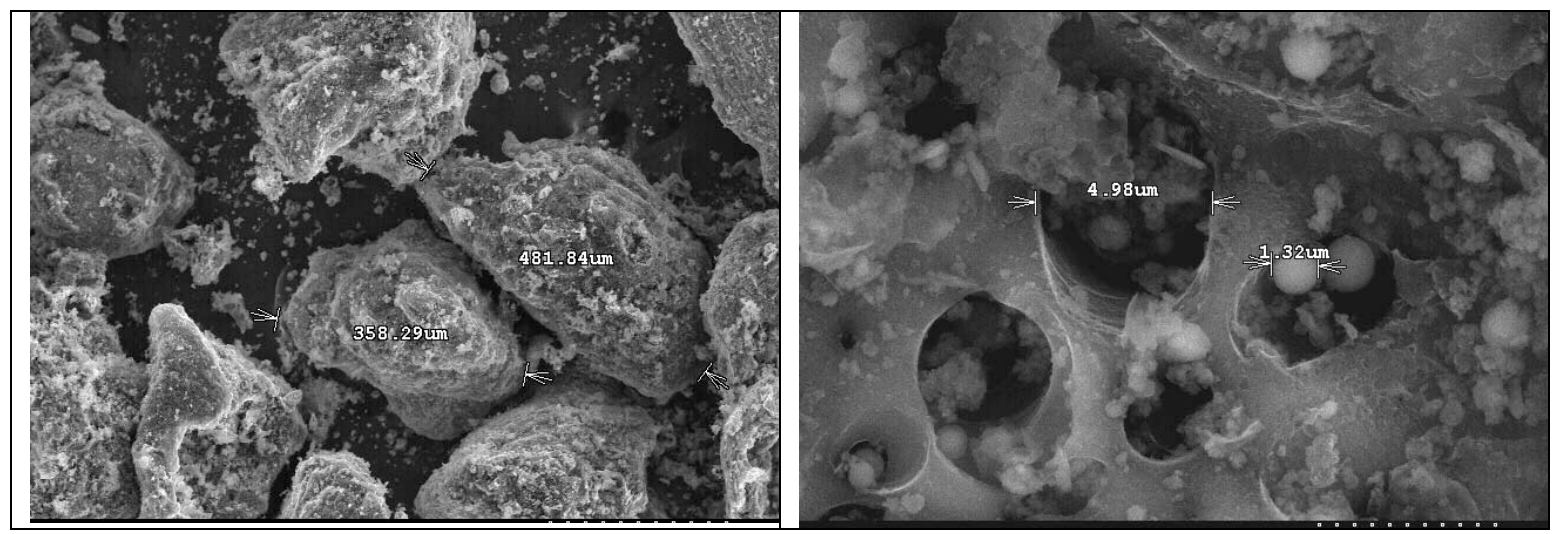

Figure 3.18. SEM images of B1(-20+80) sample prior to activation at 120 times (left) and 6,000 times (right) magnification. 


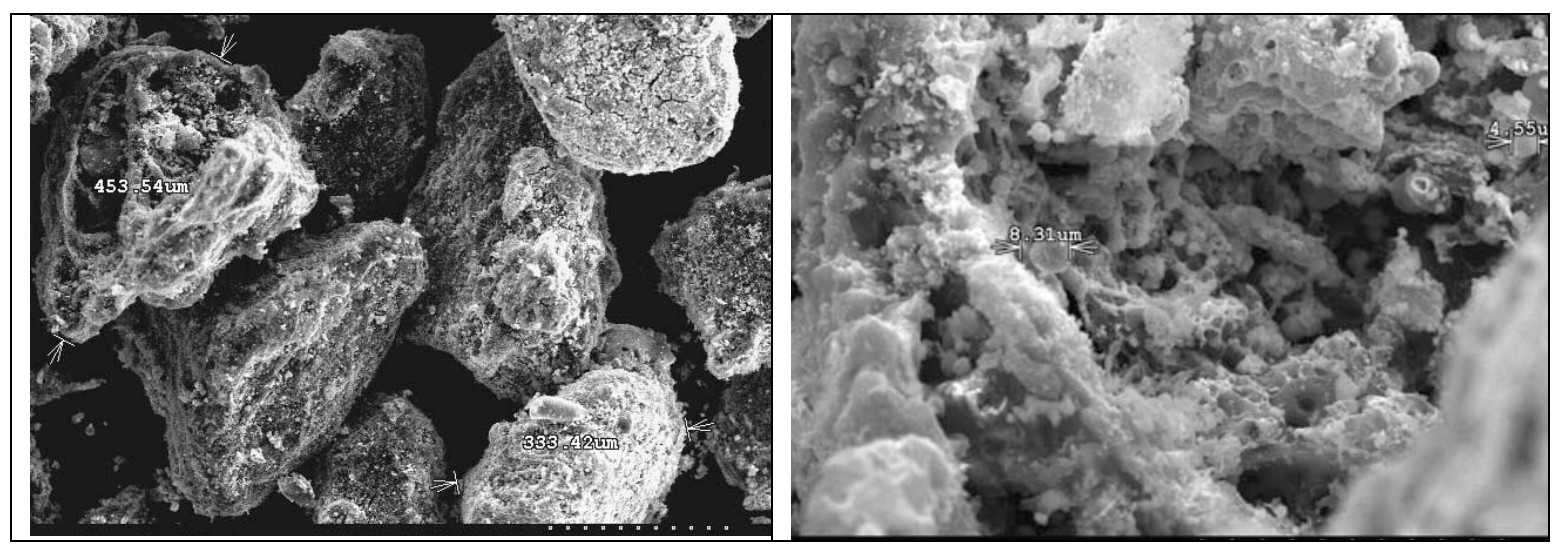

Figure 3.19. SEM images of B1(-20+80) sample activated for 90 minutes at 120 times (left) and 1,000 times (right) magnification.

The SEM images for B1(-80) are depicted in Figures 3.20 and 3.21. It appears that more spherical, smoother particles are present in the sample following activation. The particle sizes also appear to be smaller in general after the activation process. The longer the activation time, the more agglomerated particles can be observed within the sample. The images taken after 120 minutes of activation show multiple agglomerated particles, as seen in Figure 3.21. The presence of cracked or exploded particles was also observed in the longer activation time samples.

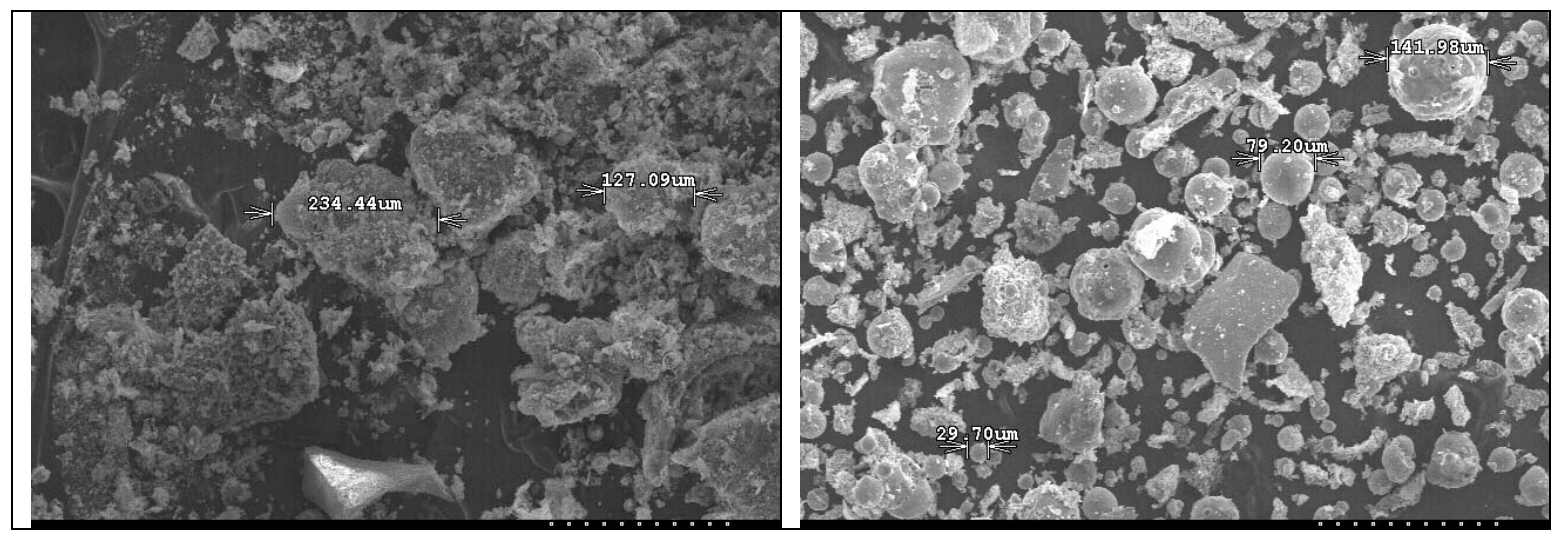

Figure 3.20. SEM images of B1(-80) sample prior to activation (left) and activated for 90 minutes (right) at 120 times magnification. 


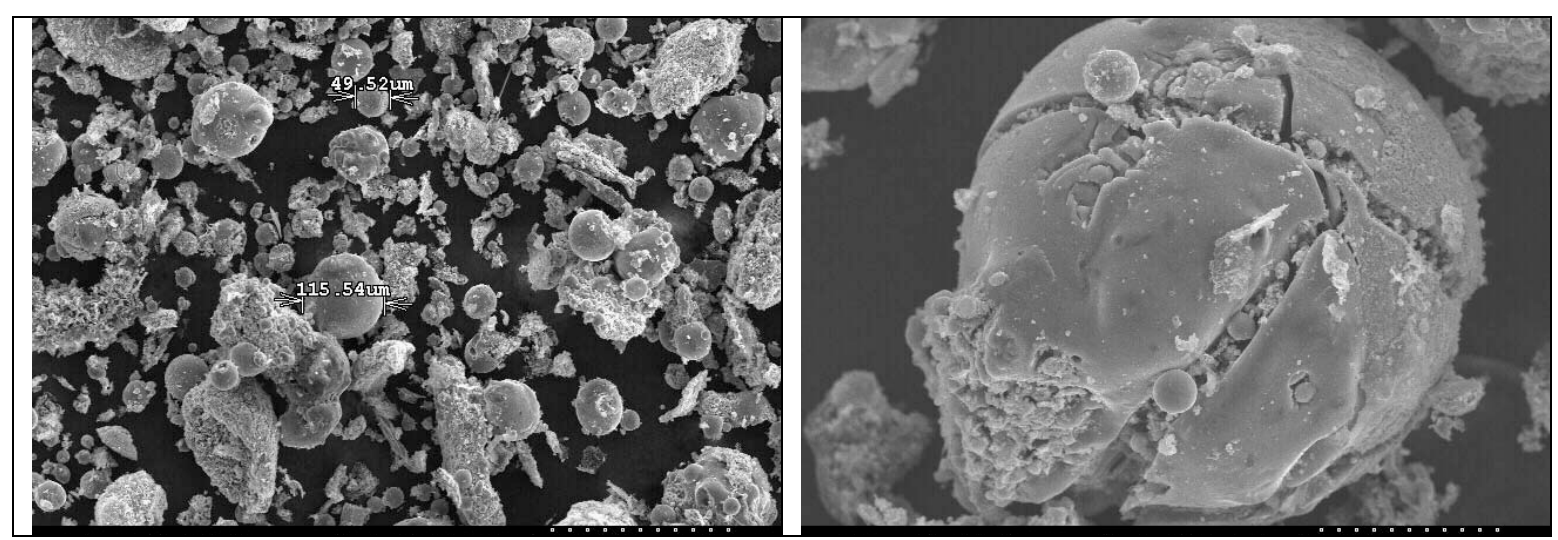

Figure 3.21. SEM images of B1(-80) sample after 120 minutes of activation at 120 times (left) and 600 times (right) magnification.

The SEM images for B2 are depicted in Figures 3.22 - 3.24. This sample had particle sizes that ranged from well over half a millimeter to only a few micrometers. It can be seen that the shape and surface of the particles changed after only 60 minutes of activation. The particles became more spherical and agglomerated and the surfaces showed signs of cracking. An interesting spherical particle was observed in the sample after 60 minutes of activation (see Figure 3.24). This particle appears to be ready to break apart or have something escape through its surface.

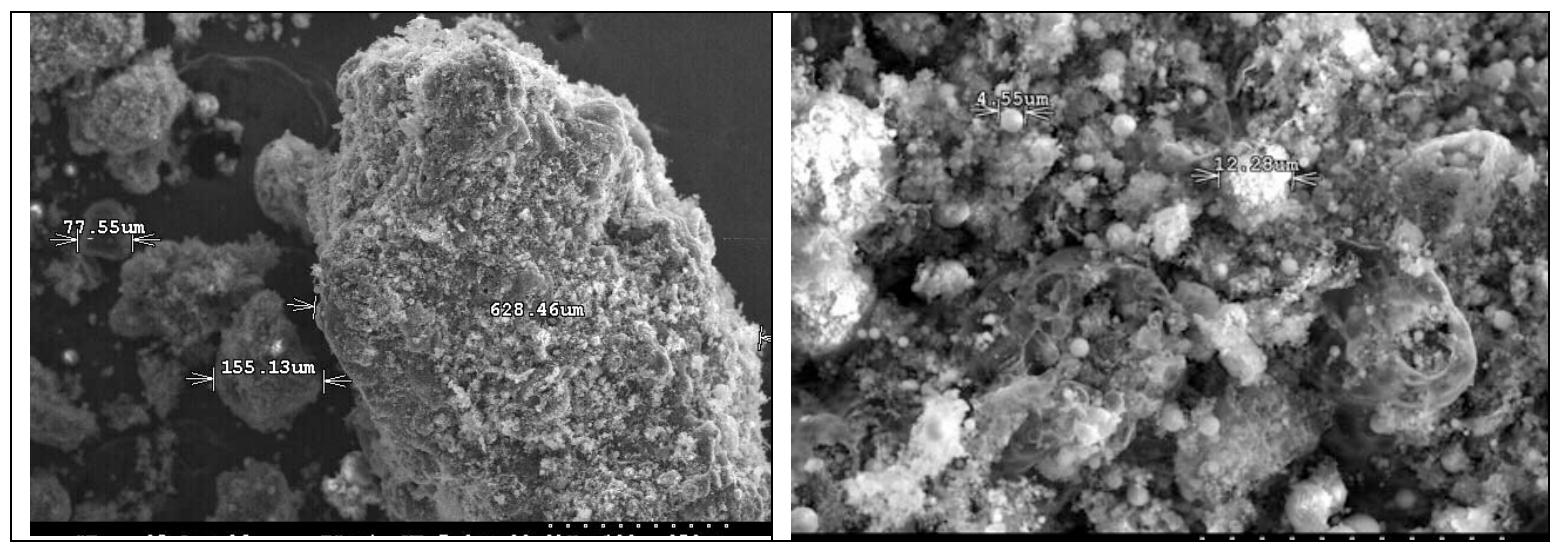

Figure 3.22. SEM images of the B2 sample prior to activation at 120 times (left) and 1,000 times (right) magnification. 

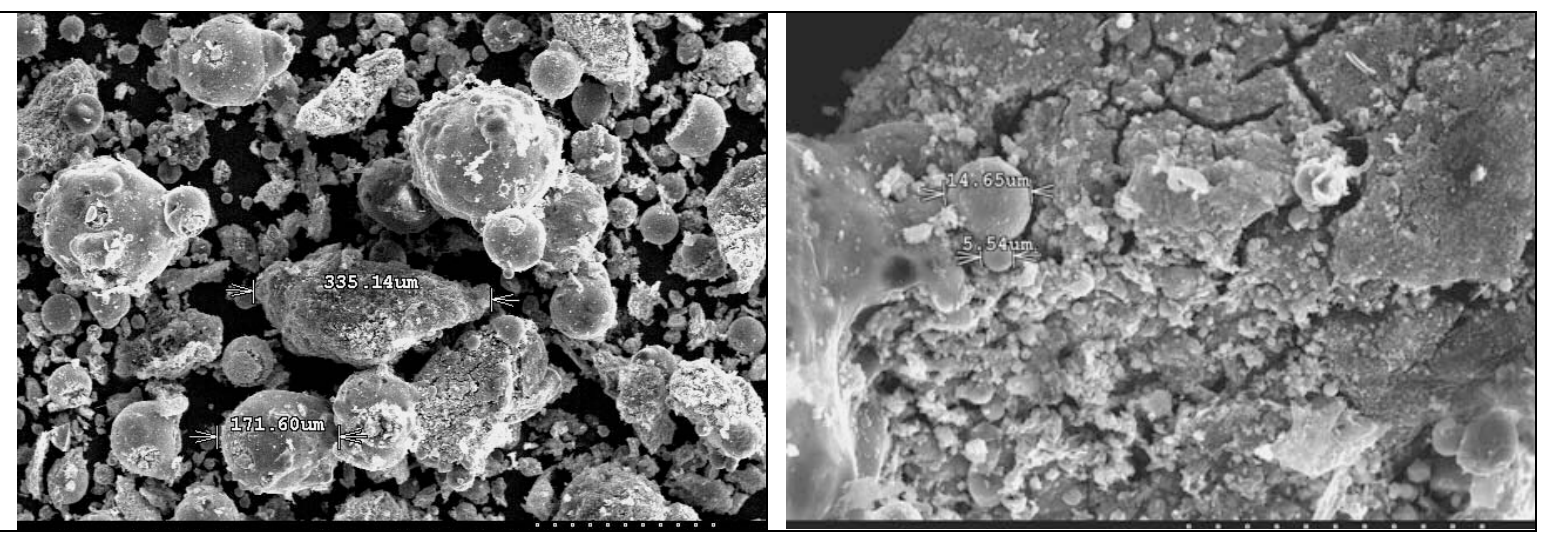

Figure 3.23. SEM images of the B2 sample activated for 60 minutes at 120 times (left) and 1,000 times (right) magnification.

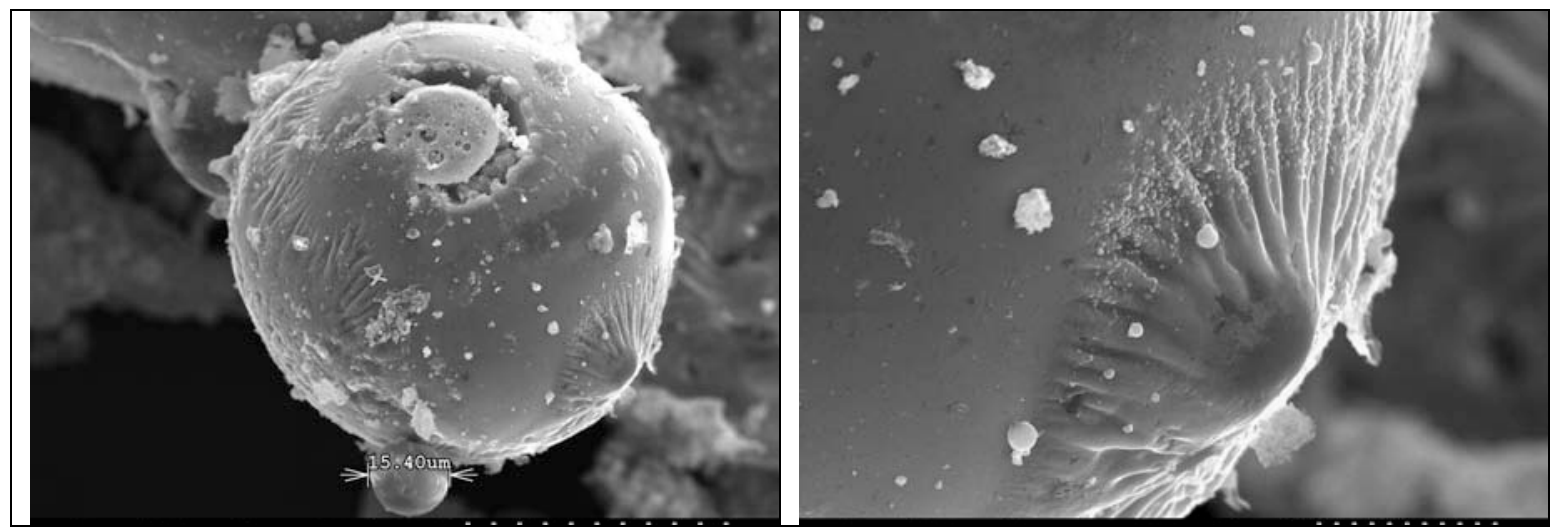

Figure 3.24. SEM images of a spherical particle in the B2 sample activated for 60 minutes at 900 times (left) and 3,000 times (right) magnification.

\subsubsection{X-ray diffraction (XRD)}

An XRD analysis was performed to determine the mineral/crystalline content of the samples. The profile for A1 $(-20+80)$ is displayed in Figure 3.25. This sample is mainly comprised of graphitic carbon, with the presence of magnetite and spinels. The unactivated sample has a shallower peak for graphitic carbon and a virtually non-existent peak for magnetite and spinels. This indicates that the structure of the sample does indeed change during the activation process to become more graphitized, with a greater presence of magnetite and spinels. 


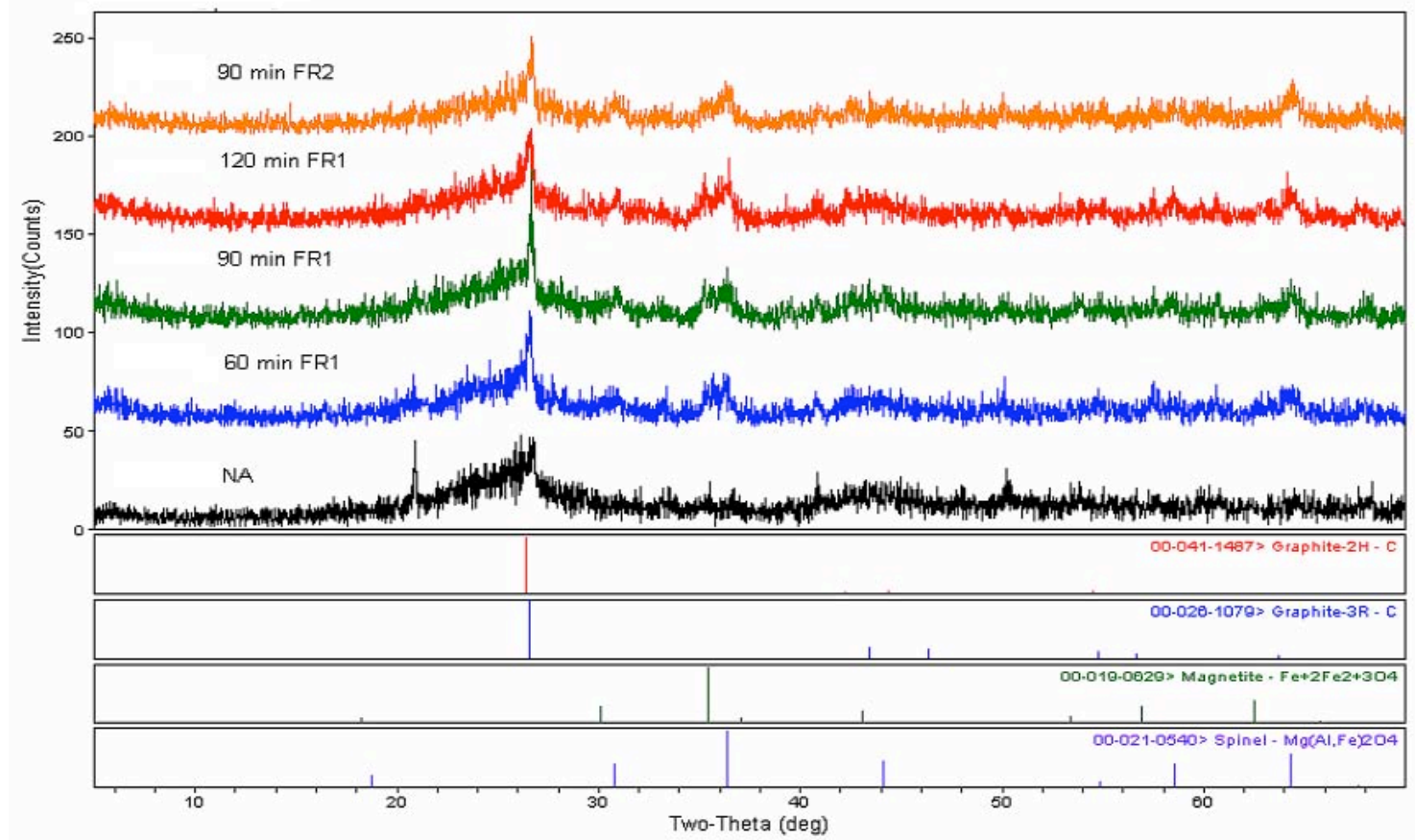

Figure 3.25. XRD profiles for A1(-20+80).

The XRD profiles for the size-separated sample B1 are show in Figures 3.26-3.28. The coarsest size fraction (+20, Figure 3.26) is composed of mainly graphitic carbon species prior to activation. The longer the activation time, the greater the shift from graphitic carbon to spinels, until at 120 minutes of activation, there is no peak corresponding to graphitic carbon. The increase in steam flow rate from 1.22 to $2.44 \mathrm{~mL} / \mathrm{min}$ shows the same trend.

The intermediate size fraction sample, B1(-20+80), exhibited a more subtle change with activation time, as shown in Figure 3.27. The inactivated sample had a bold peak corresponding to graphitic carbon species and a mostly indistinguishable peak corresponding to spinels. The spinel peaks became more apparent after activation, however, the graphitic carbon peaks were still strong in these samples. There was a slight trend of a decrease in peak height with an increase in activation time for graphitic carbon. 


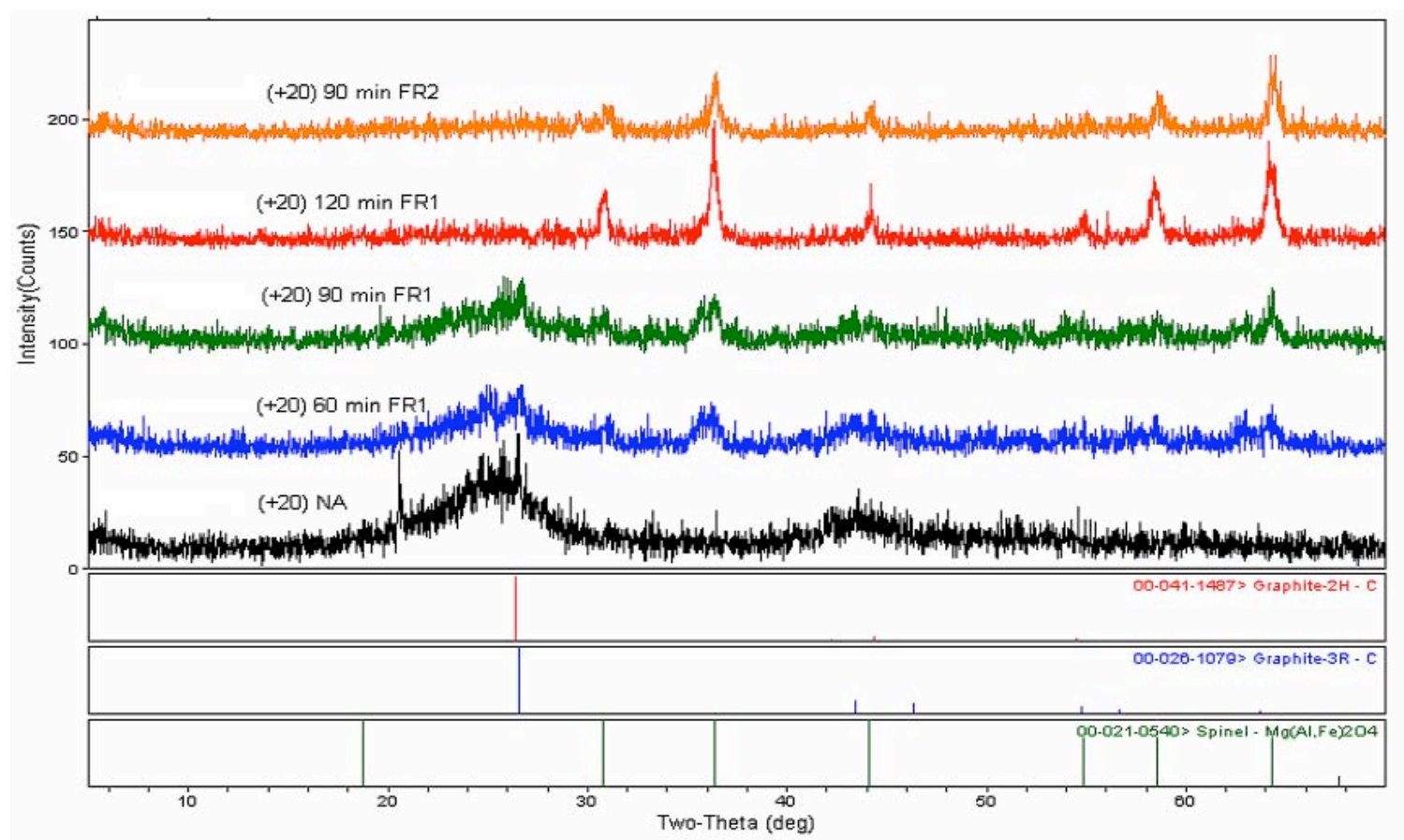

Figure 3.26. XRD profiles for B1(+20).

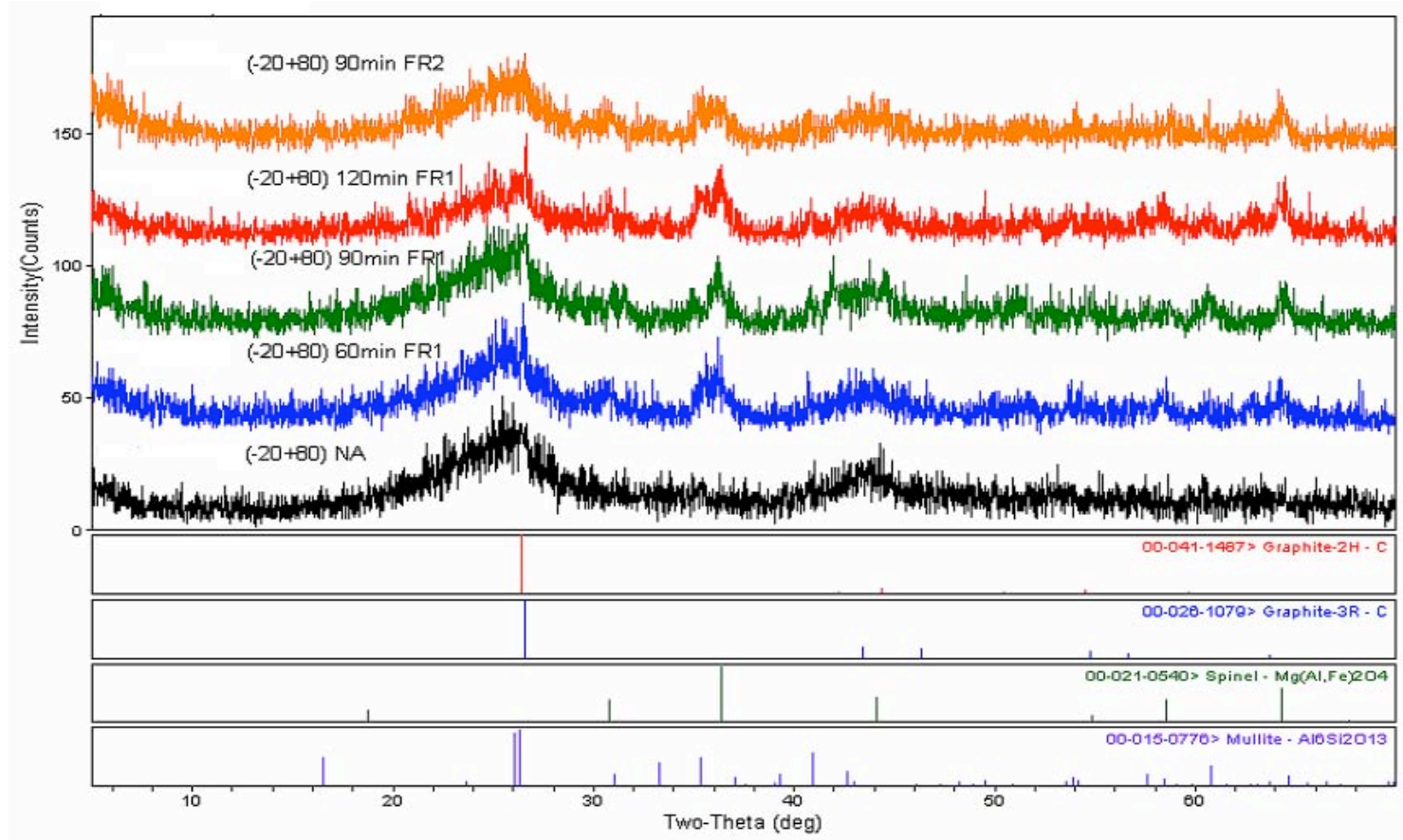

Figure 3.27. XRD profiles for B1(-20+80).

The finest size fraction, B1(-80), had the smallest peak corresponding to graphitic carbon compared to the larger particle sizes. The parent sample only had a peak corresponding to graphitic carbon, whereas the activated samples showed significant peaks that indicated the presence of spinels and magnetite. 


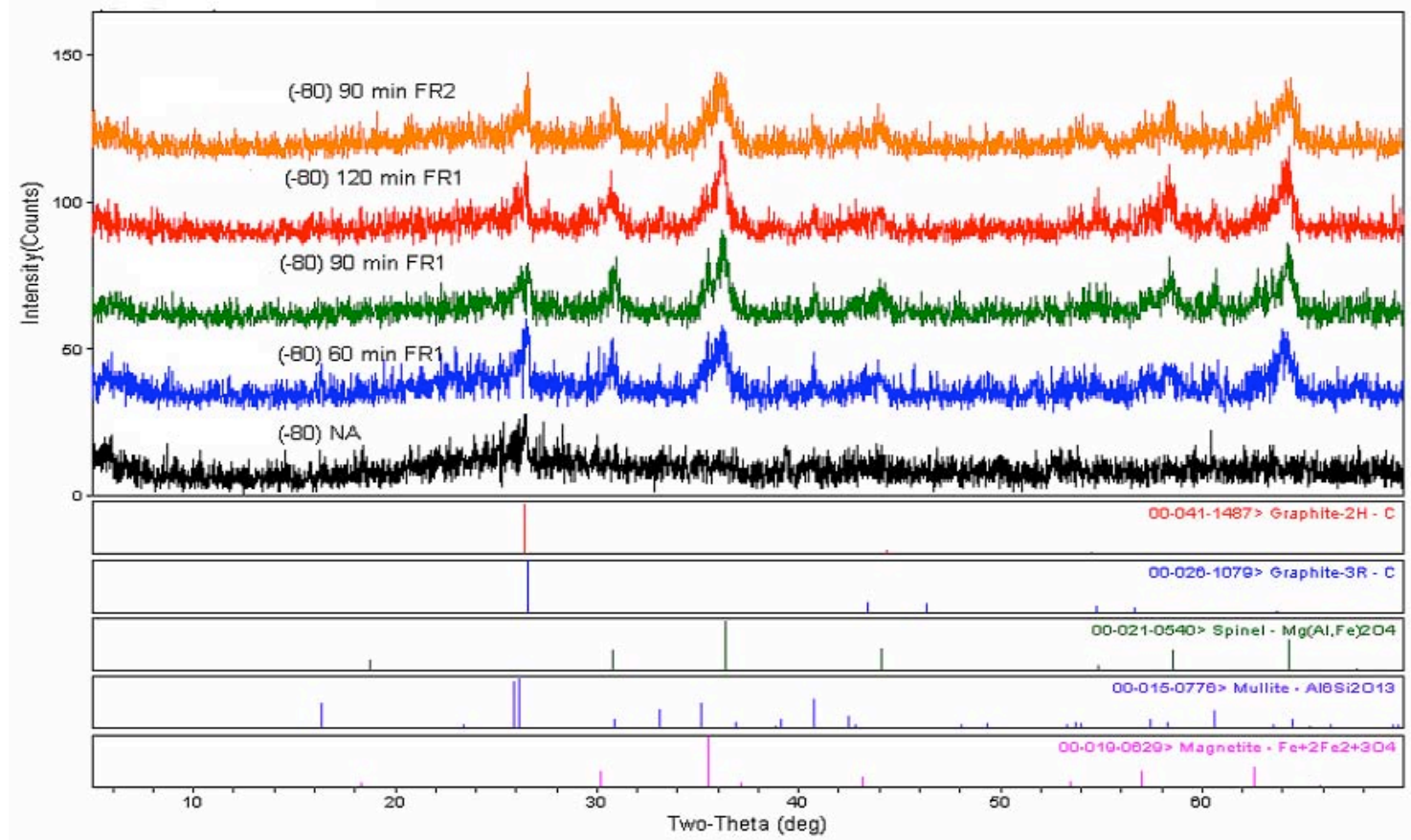

Figure 3.28. XRD profiles for B1(-80).

The B2 sample (seen in Figure 3.29) had a similar trend to that of the B1(-80) sample. The main difference between these is the intensity of the peaks corresponding to spinels in the activated samples. The B2 activated samples had larger, sharper peaks than those of B1(-80).

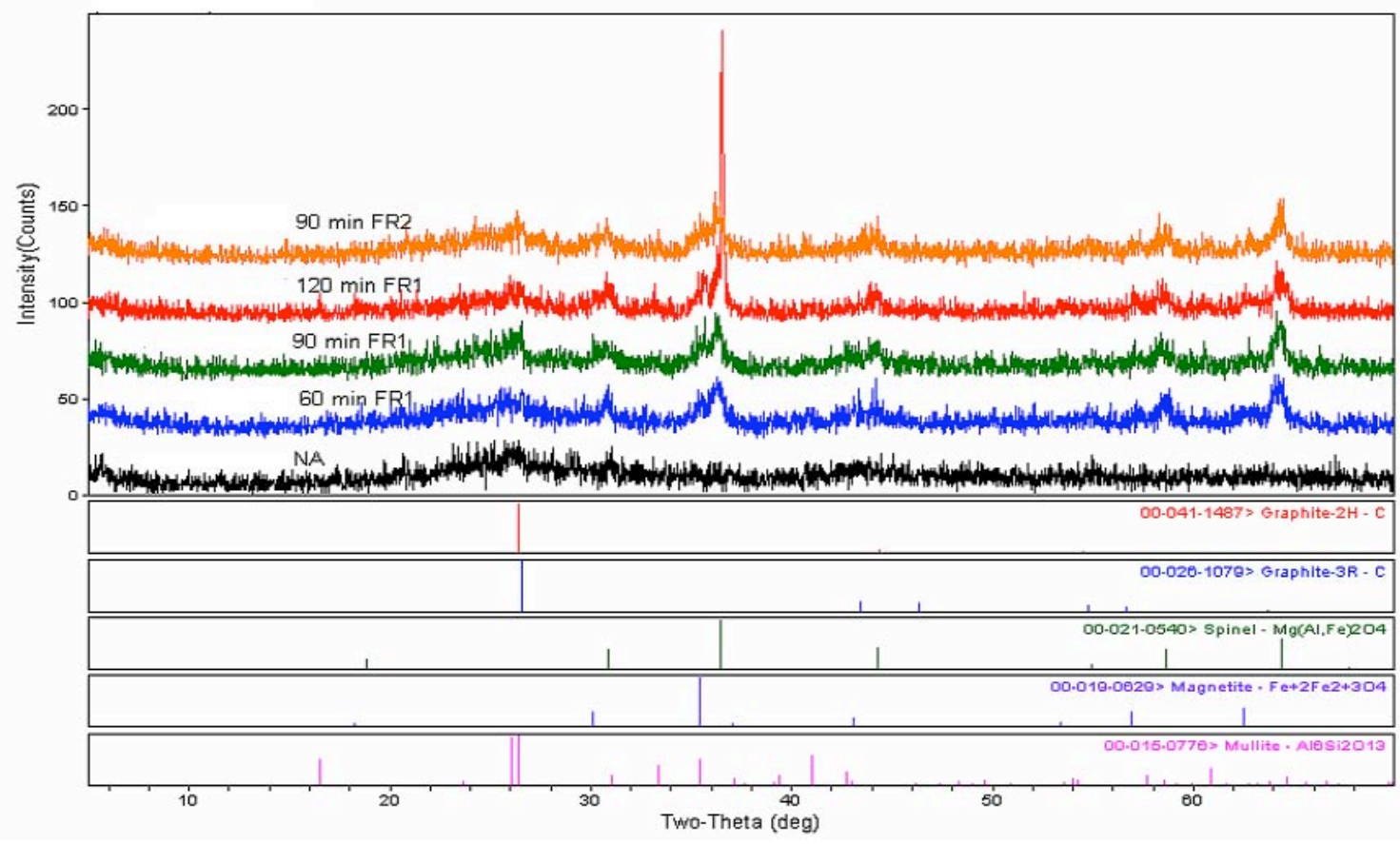

Figure 3.29. XRD profiles for B2. 


\subsection{Green Carbon Pellet Work}

Despite the use of a well-established method for forming the green carbon pellets as well as trials with different ratios of pitch-to-carbon, no successful pellets were formed. The pellets would crack and crumble immediately after removal from the mold. It is possible that the carbon samples were too oxidized to be properly wetted by the pitch. Digital (Figure 3.30) and SEM (Figure 3.31) images were taken of the failed pellets.

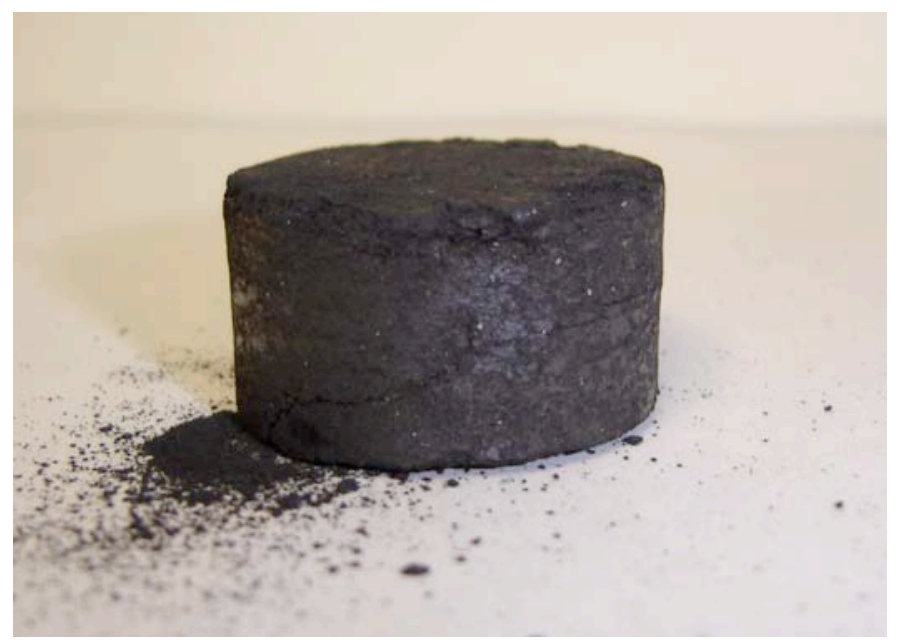

Figure 3.30. Digital photograph of a failed pellet.

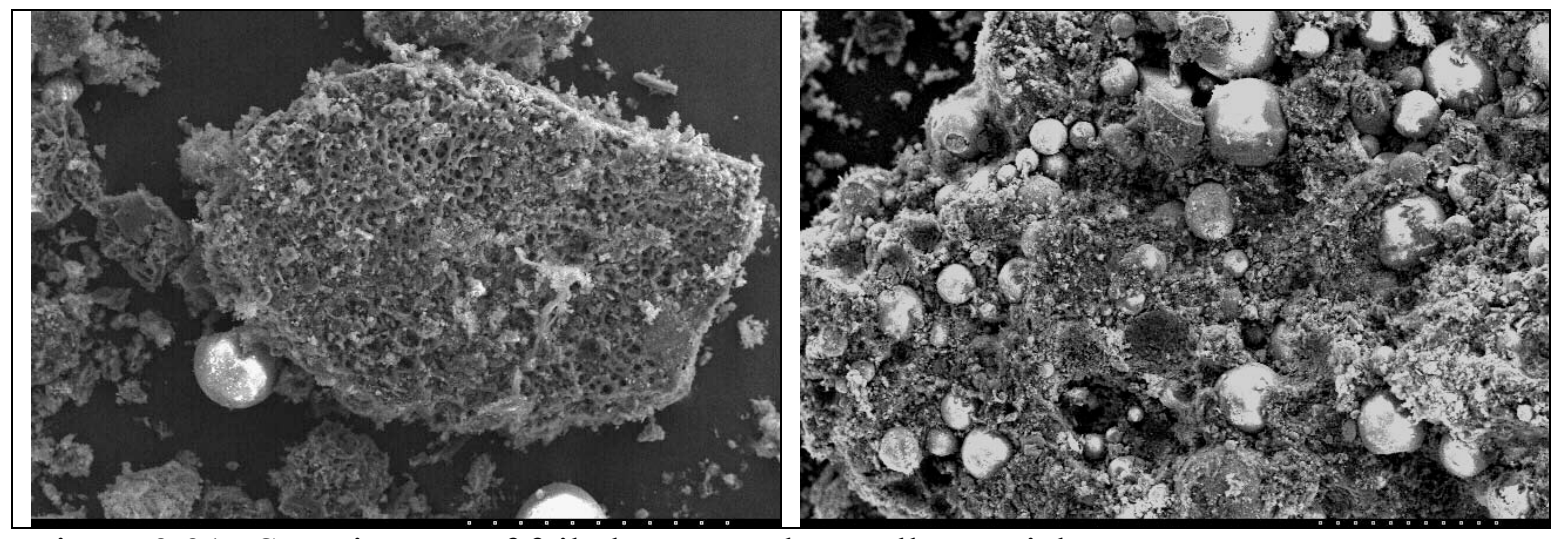

Figure 3.31. SEM images of failed green carbon pellet particles.

\section{Conclusions}

In this study, a gasification char sample was characterized using LOI, proximate, ultimate, petrographic analyses, $\mathrm{N}_{2}$ adsorption isotherms, inherent mercury determination, ICP ash chemistry, and XPS surface chemistry analyses. The sample was also tested for its ability to capture mercury vapor. It was then activated using steam at $850^{\circ} \mathrm{C}$ for different periods of time $(60,120$ and 180 minutes $)$.

The parent sample presented very low moisture, volatile matter and hydrogen, nitrogen contents, due to the devolatilization undergone during the gasification process. The LOI value of the sample was $58.71 \%$, which was higher than the value of fixed carbon. This could be because that explained by the difference between the analytical methods used to determine LOI and proximate analyses. 
The results of petrographic analysis showed that the majority of the carbon was anisotropic and was coal derived. The chars were derived from different ranks of coal, including high volatile A bituminous, and a medium volatile bituminous. The A1 sample contained a considerable amount of petroleum coke $(6.3 \%)$, which was identified as shot coke and sponge coke. Pyrolytic carbon was also distinctively higher in reflectance than coal-derived and possessed isochromatic regions suggesting alignment of the carbon parallel to the depositional surface upon which it rested.

The char sample exhibited a low inherent mercury content at $0.04 \mathrm{ppm}$ mercury, which is the amount of mercury contained within the sample as it was received. The results of the major and minor ash chemistry analysis showed the sample had moderate concentrations $\mathrm{Al}_{2} \mathrm{O}_{3}(9.5 \%$ by weight $)$ and $\mathrm{SiO}_{2}(20.9 \%$ by weight $)$ and a relatively low concentration of $\mathrm{CaO}$ at $2.6 \%$ by weight. The amount of $\mathrm{Fe}_{2} \mathrm{O}_{3}$ ( $8.4 \%$ by weight) was measured to be relatively high compared to other ash samples. Very low concentrations of $\mathrm{BaO}, \mathrm{K}_{2} \mathrm{O}$, $\mathrm{MgO}, \mathrm{MnO}, \mathrm{Na}_{2} \mathrm{O}, \mathrm{P}_{2} \mathrm{O}_{5}, \mathrm{SrO}$, and $\mathrm{TiO}_{2}$ were measured.

$\mathrm{O}_{2}$ were measured.

The XPS surface chemistry testing showed that the concentration of carbon atoms on the surface was moderate at 71.5 atomic $\%$. Nitrogen atoms on the surface were found to be at a relatively high concentration, measuring 0.7 atomic $\%$. Surface iron in the sample was also high compared to other samples at 1.7 atomic $\%$. Moderately low values were determined for calcium and phosphorus. Relatively high values were measured for oxygen and sulfur for a gasification char, with moderate values of fluorine, chlorine, and aluminum. The elements $\mathrm{Ti}, \mathrm{Na}, \mathrm{K}, \mathrm{Mg}, \mathrm{Ba}$, and $\mathrm{Co}$ were not found in any measurable surface concentrations.

The char sample was found to have very good mercury capture performance. It adsorbed $7.30 \mathrm{mg} \mathrm{Hg} / \mathrm{g}$ sorbent without experiencing breakthrough until after 45.8 hours of adsorbing. Most of the other gasification samples tested on the same mercury generation/capture rig were shown to adsorb far less mercury and experienced much shorter times to breakthrough. Overall, this char sample is an effective mercury sorbent candidate prior to any sorbent-enhancing modifications.

The initial one-step steam activation process used here increased the porosity of the sample, with the surface area increasing to $427 \mathrm{~m}^{2} / \mathrm{g}$ for activated carbon A1-180 compared to $153 \mathrm{~m}^{2} / \mathrm{g}$ for the parent sample. A parametric study was conducted to understand the effect of activation time on the properties of the resultant activated carbons. The results show that the surface area and the percentage of micropores increased with the activation time at the expense of carbon yields and reach a maximum value at 120 minutes. The total pore volume increases continuously with activation time.

Further, more comprehensive steam activation was then performed on the A1 sample, along with two additional samples, B1 and B2, with varying activation times $(60,90$, and 120 minutes) and varying steam flow rates $(1.22$ and $2.44 \mathrm{~mL} / \mathrm{min})$. Upon characterization testing following activation, including optical microscopy, 
thermogravimetric analysis, scanning electron microscopy, and x-ray diffraction, the effect of the activation process was examined.

Optical microscopy showed that, in general, steam activation appeared to result in a loss of carbon from exposed surfaces of the vitrinite-derived carbon structures with isotropic carbon being most susceptible. A slight concentration of larger anisotropic textures seemed to occur with duration of activation and a loss of the finer broken fragments (those $<10 \mu \mathrm{m}$ ) was observed. There was an increase in the size and shape of the pore spaces in the carbon structures as activation time clearly resulted in more connected porosity, although this may or may not cause an increase in measured surface area.

The TGA experiments showed the amount of weight loss in the samples over time as the surrounding temperature was increased to $900^{\circ} \mathrm{C}$. In general, the longer the activation and the higher the steam flow rate, the lesser the weight lost in each sample. This is in agreement with the loss of carbon yields with increased activation time, as discussed previously.

An analysis of the particle sizes, shapes, and surfaces as performed by an SEM analysis generally indicated an increase in agglomerated particles with increased activation time. The surfaces of the particles became more connected in porosity, with evidence of shallower, more numerous crevices (micropores) with increases in activation time.

The XRD analysis showed that the samples were comprised mainly of graphitic carbon and mullite with magnetite and spinels to a lesser degree. After activation, there was a general trend away from graphitic carbon structures and towards spinels and magnetite. This observation is in agreement with other characterization methods that indicate a decrease in carbon yields with an increase in activation time. 


\section{References}

1. Department of Energy http://www.fe.doe.gov

2. U.S. Environmental Protection Agency (EPA) Clean Air Mercury Rule (CAMR). http://www.epa.gov/air/mercuryrule/rule.htm

3. Brown, T. D.; Smith, D. N.; O'Dowd, W. J.; HargisJr, R. A., Control of mercury emissions from coal-fired power plants: a preliminary cost assessment and the next steps for accurately assessing control costs. Fuel Processing Technology 2000, 65-66, 311-341. 4. $\quad$ Maroto-Valer, M. M.; Zhang, Y.; Granite, E. J.; Tang, Z.; Pennline, H. W., Effect of porous structure and surface functionality on the mercury capacity of a fly ash carbon and its activated sample. Fuel 2005, 84, (1), 105-108.

5. $\quad$ Derbyshire, F.; Jagtoyen, M.; Thwaites, M., Porosity in Carbon. Halsted Press: New York, Toronto, 1995.

6. Maroto-Valer, M. M.; Taulbee, D. N.; Hower, J. C., Energy Fuels 1999, 13, 947.

7. Kruk, M.; Jaroniec, M.; Gadkaree, K. P., Journal of Colloid and Interface Science 1997, 192, 250.

8. Perry, R. E. Process Assessment (Room B14 Laboratory L3); University of Nottingham - School of Chemical, Environmental, and Mining Engineering: Nottingham, UK, 2005; pp 1-6.

9. Perry, R. E., Development of a Rig to Measure Mercury Removal on Novel Sorbents. In Nottingham Fuel \& Energy Centre, The University of Nottingham: Nottingham, UK.

10. Ahmadpour, A.; Do, D. D., Carbon 1996, 34, 471-479.

11. Lu, Z. Characterization and Steam Activation of Unburned Carbon in Fly Ash. Pennsylvania State University, University Park, 2003.

12. Zhang, Y.; Lu, Z.; Maroto-Valer, M. M.; Andresen, J. M.; Schobert, H. H., Comparison of High Unburned Carbon Fly Ash from Different Combustor Types and Their Steam Activated Products. Energy Fuels 2003, 17, 369-377.

13. Sloss, L. L.; Smith, I. M.; Adams, D. M. B., Pulverized Coal Ash Requirements for Utilization. In IEA Coal Research: London, 1996.

14. Maroto-Valer, M. M.; Taulbee, D. N.; Hower, J. C., Characterization of differing forms of unburned carbon present in fly ash separated by density gradient centrifugation. Fuel 2001, 80, (6), 795-800.

15. Markley, B. M. Examining the Correlations Between Fly Ash and Gasification Char Properties and Mercury Uptake Capacities. The Pennsylvania State University, University Park, 2006.

16. Brunauer, S.; Deming, L. S.; Deming, W. S.; Teller, E. J., J. Amer. Chem. Soc. 1940, 62, 1723.

17. Gregg, S. J.; Sing, K. S., Adsorption, Surface Area, and Porosity. Academic Press, Inc.: London, 1982.

18. Rodríguez-Mirasol, J.; Cordero, T.; Rodríguez, J. J., Carbon 1993, 31, (1), 87.

19. Bansal, R. C.; Donnet, J.; Stoeckli, F., Active Carbon. Marcel Dekkar, Inc.: New York, Basel, 1988. 


\section{CAER - PSU JOINT CONCLUSIONS}

Two gasifier slag carbons, A1 and A3, had exceptional Hg capture capacities. Both of these slags come from plant A which feeds primarily coal but also varying amounts of petcoke. Sample A1 was analyzed by PSU for its petcoke derived carbon which was 6\%. None of the other samples in this study from either plant A or B were analyzed for their portion of petcoke derived carbon. Therefore, the contributions of petcoke carbon to $\mathrm{Hg}$ adsorption could not be determine and would require obtaining multiple samples with varying amounts of petcoke carbon. A1 was tested for $\mathrm{Hg}$ adsorption independently by CAER and PSU and found to have very good Hg capture performance. A1 and A3 were chemically and physically characterized by CAER and PSU. The data indicated that good Hg adsorption capacity for these carbons is complex. Some of the chemical characteristics of these gasifier slag carbons suggested their carbon and sulfur content, the high oxygen content, the presence of either halogens or $\mathrm{SO}_{4}{ }^{-2}$ or both were important. The carbon contents of A1 and A3 were 46 and 77\% respectively and yet had nearly the same Hg adsorption capacity. This suggests that carbon content is not the sole determinant of $\mathrm{Hg}$ capture potential. High $\mathrm{Cl}^{-}, \mathrm{F}^{-}, \mathrm{Br}^{-}$and oxygen in these sample may be important in Hg capture since they have been implicated in the oxidation of $\mathrm{Hg}^{0}$ to $\mathrm{Hg}^{+2}$ which is easier to remove from gas streams. $\mathrm{PO}^{-2}$ was high in both good Hg sorbents since it has not been implicated previously in Hg adsorption, more work needs to be done to prove its relativity to the capture of $\mathrm{Hg}$. Impregnation of a sorbent with $\mathrm{PO}^{-2}$ and subsequent $\mathrm{Hg}$ adsorption testing would prove useful in determining its significance in Hg capture by carboneous materials.

XPS surface chemistry testing at PSU showed that the concentration of carbon atoms on the surface was moderate at 71.5 atomic percent. Nitrogen atoms on the surface were found to be at a relatively high concentration, measuring 0.7 atomic percent. Relatively high values were measured for oxygen and sulfur for A1, with moderate values of fluorine, chlorine, and aluminum, suggesting that these sample have mineral surface component which maybe as important as carbon for good Hg adsorption. The surface areas of both good Hg capturing carbons, A1 and A3, were greater than $100 \mathrm{~m}^{2} / \mathrm{g}$. However, the Hg capture capacities of A1 and A3 were higher than a specialty carbon developed specifically for Hg adsorption. The surface area of the specialty carbon was $570 \mathrm{~m}^{2} / \mathrm{g}$ suggesting high surface area may also not be the determining factor in good $\mathrm{Hg}$ adsorption. Similarly another gasifier carbon, B, had higher surface area than A1 and A3 and did not adsorb Hg. However, some surface area must be required since sample A2 with a surface area of less than $25 \mathrm{~m}^{2} / \mathrm{g}$ did not absorb Hg. The pore volumes of A1 and A3 were substantially greater than A2 and B. The greater pore volume was attributed to meso and macropores. Previous work suggested that these pores were important in $\mathrm{Hg}$ adsorption (Rubel, et. al., 2005). The conclusion for both these carbons was that they were excellent $\mathrm{Hg}$ adsorbing carbons without modification. All other gasifier carbons studied during this project were poor Hg sorbents, indicating that while the determinant factors for a char's suitability as an Hg sorbent are a complex combination of both physical and chemical characteristics, some porosity is required to attain any significant Hg capture utility. Therefore, gasifier slag carbons which exhibit little or no meso/macroporosity will not be suitable for Hg capture (although further work is needed to determine the exact nature of the specific pore size distribution required for optimal 
capture). However, the presence of such porosity is not a sufficient indicator of $\mathrm{Hg}$ capture potential without suitable chemical binding sites being present.

Part of the work on $\mathrm{Hg}$ adsorption on the gasifier carbons was to study the binding sites of the Hg on the gasifier carbons. Mercury, sulfur and chlorine XAFS spectra were collected for two slag carbon samples of differing Hg contents, A1 (good sorbent) and A2 (poor sorbent), and a control sample (A1 with no Hg). Analysis of the Hg XAFS data suggested that the Hg is bound predominantly to S; however, it should be noted that $\mathrm{Cl}$ is not eliminated by the data because of its proximity to sulfur in the periodic table and its similar X-ray scattering properties. In addition, the data indicated that the $\mathrm{Hg}$ coordination and bond length to sulfur were significantly reduced compared to that in cubic HgS. However, this may be a result of the mercury being bound at the surface of the sorbent rather than in a well-defined crystal structure. Sulfur and chlorine XANES spectra indicated that the sulfur and chlorine speciation between the two gasifier carbon samples were very different. In the A2 sample, sulfur is present mostly as metal sulfides, whereas the A1 carbon contained elemental sulfur, thiophene and sulfate forms as major forms and very little, if any, sulfur as metal sulfide. In thiophene sulfur is bound in an aromatic ring and not very reactive, suggesting elemental sulfur and sulfate forms are important in Hg adsorption. Analysis of the chlorine XANES data indicated the possible presence of organochlorine compounds. These data strongly suggest that sulfur and chlorine are important in binding $\mathrm{Hg}$ to the carbons.

Several of the slag samples, B (-20+80), B (soot), and B (filter cake), adsorbed $40 \%$ of the $\mathrm{NO}_{\mathrm{X}}$ adsorbed by the commercial activated carbon, HGR. The adsorption of Hg on sample A1 (-20+80) increased its $\mathrm{NO}_{\mathrm{X}}$ adsorption capacity by $27 \%$. This indicated that $\mathrm{Hg}$ and $\mathrm{NO}_{\mathrm{X}}$ have different binding sites on the carbon and the use of the good $\mathrm{Hg}$ adsorbing slags for $\mathrm{Hg}$ control could have an added benefit of further reducing $\mathrm{NO}_{\mathrm{X}}$ emissions of the primary $\mathrm{NO}_{\mathrm{X}}$ emissions control technology being used by a power plant.

$\mathrm{NO}_{\mathrm{X}}$ adsorption on the slag carbons correlated with the BET SA and micropore volumes of the samples. This is consistent with work with has shown that $\mathrm{NO}_{\mathrm{X}}$ adsorption on activated carbons is mainly in micropores with the optimum pore size of approximately 11 angstroms. The Hg-laden A1 (-20+80) sample had an increased in SA, total pore volume and micropore volume over the A1 sample without Hg. BET SA, total pore volume and the micropore volume increased from 109 to $199 \mathrm{~m}^{2} / \mathrm{g}, 0.068$ to 0.081 $\mathrm{cm}^{3} / \mathrm{g}$ and 0.25 to $0.34 \mathrm{~cm}^{3} / \mathrm{g}$ respectively when $\mathrm{Hg}$ was loaded onto the sample. Previous work at the CAER has suggested that partial filling of a meso and macropores with Hg would produce some smaller spaces (micropores or equivalent spaces) for $\mathrm{NO}_{\mathrm{X}}$ adsorption (Rubel, et. al., 2005). The increase in surface area, pore volume, and micropore volume observed for Hg-laden A1 support this conclusion. Additionally published work has strongly suggested that $\mathrm{Hg}$ is adsorbed as $\mathrm{Hg}^{++}$even from simulated flue gas containing only $\mathrm{Hg}^{0}$ and that it is ionically bound to the surface of the sorbent (Huggins, et. al., 2003). The work at the CAER agrees with chemical bonding of Hg on sorbents since it is only released above $300^{\circ} \mathrm{C}$ during heating (Rubel, et. al., 2003). The ionic binding of $\mathrm{Hg}$ to the surface of pores will result in changes in the characteristics of the pores which the data shows makes the pore more suitable for $\mathrm{NO}_{\mathrm{X}}$ adsorption. From this, it can be concluded that these materials are suitable as simultaneous $\mathrm{Hg}$ and $\mathrm{NOx}$ 
sorbents, with synergistic enhancement of NOx capture due to apparent filling of the largest pores by captured Hg species.

Several of the gasifier carbons were thermally activated at PSU and two were chemically activated at CAER. The thermally treated carbons, A1(-20+80), B(-20+80), and $B(+20)$ were successfully activated with BET SA increasing from 109 to 220, 142 to 330 , and 99 to $170 \mathrm{~m}^{2} / \mathrm{g}$ for $\mathrm{A} 1(-20+80), \mathrm{B}(-20+80)$, and $\mathrm{B}(+20)$ respectively. Chemical activation at the CAER of samples A1(-20+80) and B(-20+80) resulted in a loss of surface area and a breakdown of the carbon matrix. Characterization of the activated gasifier carbons included optical microscopy, thermogravimetric analysis, scanning electron microscopy, and x-ray diffraction at PSU and BET SA and pore size distribution at the CAER (results above). While these results indicate that these chars can be enhanced by classical activation approaches, none of the resultant materials exhibit significantly high surfaces areas to make them competitive with commercially prepared activated carbons.

PSU optical microscopy results showed that, in general, steam activation appeared to result in a loss of carbon from exposed surfaces of the vitrinite-derived carbon structures with isotropic carbon being most susceptible. A slight concentration of larger anisotropic textures seemed to occur with duration of activation and a loss of the finer broken fragments (those $<10 \mu \mathrm{m}$ ) was observed. There was an increase in the size and shape of the pore spaces in the carbon structures as activation time clearly resulted in more connected porosity, although this may or may not cause an increase in measured surface area.

The TGA experiments showed the amount of weight loss in the samples over time as the surrounding temperature was increased to $900^{\circ} \mathrm{C}$. In general, the longer the activation and the higher the steam flow rate, the lesser the weight lost in each sample. This is in agreement with the loss of carbon yields with increased activation time.

An analysis of the particle sizes, shapes, and surfaces as performed by an SEM analysis generally indicated an increase in agglomerated particles with increased activation time. The surfaces of the particles became more connected in porosity, with evidence of shallower, more numerous crevices (micropores) with increases in activation time.

The XRD analysis showed that the samples were comprised mainly of graphitic carbon and mullite with magnetite and spinels to a lesser degree. After activation, there was a general trend away from graphitic carbon structures and towards spinels and magnetite. This observation is in agreement with other characterization methods that indicate a decrease in carbon yields with an increase in activation time. Likely, the overall low surface areas achievable result from a lack of reactive carbon in the structure. This conclusion is further supported by the observed loss of surface area when very harsh chemical activation was used, where, once the less reactive carbon is removed, the structure collapses and porosity is lost.

All PSU thermally activated slag samples A1 (-20+80), B (-20+80), B (soot), B ($80)$, and B (+20) were tested for Hg adsorption potential at the CAER. The A1 slag which had high $\mathrm{Hg}$ adsorption capability lost $94 \%$ of its $\mathrm{Hg}$ adsorption capacity on activation. This again suggested that factors other than higher surface area and pore volume were important in Hg adsorption. Mineral content of the slags may play an important role in Hg capture. Except for the B (-80) samples, the other B activated 
carbons had increased $\mathrm{Hg}$ adsorption at the 60 and 90 min thermal activation times. Steam activation at 90 min also improved $\mathrm{Hg}$ adsorption in all B slag carbons except for B (-20+80). However, the increased Hg adsorption was still much lower than the untreated sample A1. There were no correlations between BET SA or pore volumes and Hg adsorption capacities again indicating that some SA and pore volume were required but were not the determining factors in Hg adsorption.

The activated samples of two gasifier slags, A1 (-20+80) and B (-20+80), were also tested for their $\mathrm{NO}_{\mathrm{X}}$ adsorption capacities. It was expected that activation would increase the $\mathrm{NO}_{\mathrm{X}}$ adsorption capacities but this was not the case. Most likely this was because the increase in the SA and pore volume of these samples was mostly related to increases in mesopore volume and not micropores. This was supported by the fact that no correlations were found between $\mathrm{NO}_{\mathrm{X}}$ adsorption and BET SA, and pore volumes for the activated samples.

As a final test of the use of gasifier slag carbons for $\mathrm{Hg}$ adsorption, three of the slags, $\mathrm{A} 1, \mathrm{~A} 2$, and $\mathrm{B}$, were tested in a simulated flue gas containing $\mathrm{NO}$ and $\mathrm{SO}_{2}$. All were compared at the same particle size distribution. As with $\mathrm{Hg}$ adsorption on these samples in air, the A1 slag out performed the other slag carbons. However, the presence of $\mathrm{NO}$ and $\mathrm{SO}_{2}$ in the adsorption gas decrease its adsorption capacity $40 \%$. While the results are encouraging for the use of some gasifier carbons for the control of $\mathrm{Hg}$ emissions from electric power plants, they do indicate more work is needed to better define the interactions between Hg adsorption and the presence of other flue gas components.

The leaching testing of $\mathrm{Hg}$ loaded and virgin slag carbon indicated that the $\mathrm{Hg}$ captured by the slag was very stable and would not be released to the ground water on disposal. At the conditions studied less than $4 \mathrm{e}-4 \%$ of the Hg captured by the slag was released.

A cursory evaluation of the +20 mesh fraction of gasifier slag A1 and B indicated a potential for use as a pozzolanic concrete admixture and should be tested in future work. The pozzolanic reactivity of the gasifier slags should be verified using the protocol for fly ash, ASTM C 311. Additionally the slags should be tested for use as a coarse aggregate by comparing the mechanical strength compared with conventional aggregates. Since the slags contain iron sulfide a test slab should also be prepared, cured and monitored for staining.

The study of the potential use of the gasifier slag carbons as polymer fillers indicated no obvious benefit to adding slag carbon A3 to a polymer system for the purposes of mechanical reinforcement. However, larger amounts of gasifier slag A3 positively affected the thermal diffusivity of both polypropylene and a thermoplastic elastomer (TPE-A), similar to enhancements seen with the addition of pyrolytic carbon blacks to these polymers. However, a large amount of A3 increased the electrical conductivity of TPE-A only, and did not show similar behavior in polypropylene. It can be concluded that a suitable surface modification would be required to achieve suitable wetting and dispersion characteristics of the char into polypropylene as compared to the more substituted TPE. With little observable mechanical enhancement resulting from the use of the char as fillers, the addition of such gasifier carbons for strictly thermal or electrical enhancement is unlikely to garner much attention, especially as the higher 
additions of filler caused undesirable changes in the polymer color and texture when molded.

Similarly, the char was not found to be acceptable as a replacement for petroleum coke particles as filler in carbon bodies. Besides a significantly higher ash content than is usually acceptable in such materials as anodes, electrodes or friction braking surfaces, the chars are too heavily oxidized to wet properly with binder pitch. This lack of wetability results in mechanical failure once removed from the mold due to pitch starved regions within the carbon body and fracture along the rims formed around the more glassy particle inclusions.

The results from this work indicated that certain gasifier slag carbons have the potential to capture $\mathrm{Hg}$ and $\mathrm{NO}_{\mathrm{X}}$ from combustion flue gas. Two slag carbons performed as well as a commercial activated carbon prepared specifically for Hg adsorption. It did so without further treatment making it an inexpensive alternative to the commercial activated carbon. The potential for the same gasifier slag carbon to aid in the removal of $\mathrm{NO}_{\mathrm{X}}$ appears promising. Additional characterization of the differences between slag carbon that have good versus poor adsorption is needed with particular emphasis on their petcoke carbon, halogen, forms of sulfur, and $\mathrm{PO}^{-2}$ contents, A better understanding of how other combustion gases affect the adsorption of $\mathrm{Hg}$ and $\mathrm{NO}_{\mathrm{X}}$ on gasifier slag carbons is also needed. This would require a series of adsorption experiment using different concentration of combustion gases such as $\mathrm{SO}_{2}, \mathrm{NO}_{\mathrm{X}}, \mathrm{CO} / \mathrm{CO}_{2}, \mathrm{HCl}$, and $\mathrm{H}_{2} \mathrm{O}$. Considering the benefits for the use of gasifier slag carbon as an inexpensive, onsite available, sorbent for $\mathrm{Hg}$ and $\mathrm{NO}_{\mathrm{X}}$, further work in this area is warranted. 